\title{
Estimation of the Direction of Arrival of Unknown Number of Sources Using Compressive MMV and Sparse Array Geometry
}

\section{Sayed Zeeshan Asghar}

School of Electrical and Electronic Engineering

A thesis submitted to Nanyang Technological University in partial fulfillment of the requirement for the degree of Doctor of Philosophy 



\section{Acknowledgments}

Firstly, I thank my supervisor Assoc. Prof. NG Boon Poh for his immense guidance, support and continuous encouragement.

I am also grateful to my team mates of Information System Research Laboratory, particularly Vinod Veera Ready, for their help in various ways including fruitful discussions on various subjects.

I would also like to acknowledge the support and patience of my wife, Farheen during the course of my studies and the joy brought to us by the little wonder, Ali.

Finally, I would like to dedicate this thesis to my parents. 



\section{Contents}

Acknowledgments $\quad$ i

Table of Contents iii

Summary viii

List of Figures $\quad$ xi

List of Tables $\quad$ xviii

List of Acronyms $\quad$ xix

List of Symbols $\quad$ xix

Chapter 1 Introduction 1

1.1 Background ........................ 1

1.2 Objectives and Motivation . . . . . . . . . . . . . . . 3

1.3 Major Contributions of the Thesis . . . . . . . . . . . . . 6

1.4 Organization of the Thesis . . . . . . . . . . . . . . 7

$\begin{array}{lll}\text { Chapter } 2 & \text { Review of Concepts } & 9\end{array}$

2.1 Maxwell's Theory of Electromagnetism . . . . . . . . . . . . . 9

2.2 Array and Steering Vector . . . . . . . . . . . . . . . . 11 
2.3 Array Signal Processing . . . . . . . . . . . . . . . . 14

2.4 Narrowband DOA Estimation . . . . . . . . . . . . . . . . 14

2.4.1 Beamforming ................... 15

2.4.2 The MVDR Algorithm . . . . . . . . . . . . . . 15

2.4.3 The MUSIC Algorithm . . . . . . . . . . . . . . . . . . 16

2.4.4 The MUSIC-like Algorithm . . . . . . . . . . . . . . 17

2.5 Broadband DOA Estimation . . . . . . . . . . . . . . 18

2.5.1 Incoherent Signal Subspace Method (ISSM) . . . . . . . . . 19

2.5.2 Coherent Signal Subspace Method (CSSM) . . . . . . . . . . 19

2.6 Compressive Sensing $(\mathrm{CS})$. . . . . . . . . . . . . . . 21

2.6.1 Simultaneous Orthogonal Matching Pursuit (SOMP) . . . . 24

2.6.2 MUSIC with SOMP Support ............ 25

\section{Chapter 3 Recursive MUSIC-Like Algorithm for DOA Estima-} tion of Narrowband Sources $\quad 26$

3.1 Introduction . . . . . . . . . . . . . . . . . . . 26

3.2 Multiple Measurement Vectors . . . . . . . . . . . . . . . . . . . 28

3.3 Recursive MUSIC-like (RML) Algorithm . . . . . . . . . . . . . . . 32

3.3.1 Stoppage Criterion for RML Algorithm . . . . . . . . . . 37

3.3.2 Bounds for the Optimization Parameter $\beta$. . . . . . . . . 40

3.3.3 When the Approximation $\boldsymbol{R} \approx \boldsymbol{\Sigma}_{y y}$ Becomes Invalid Because of Very Few Snapshots . . . . . . . . . . . . . . . . 44

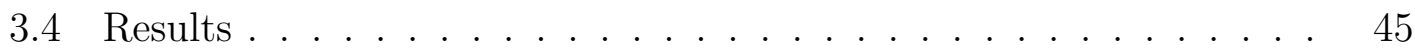

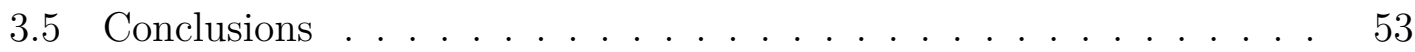


Chapter 4 Recursive MUSIC-Like Algorithm for DOA Estimation of Broadband Sources $\quad 54$

4.1 Introduction . . . . . . . . . . . . . . . . 54

4.2 Broadband Approaches to DOA Estimation . . . . . . . . . . . 55

4.2.1 Taylor Series Expansion Model . . . . . . . . . . . . . 57

4.2.2 Frequency Focusing Model (Robust Auto-Focusing Scheme) $[36] \ldots \ldots \ldots \ldots \ldots$

4.2.3 Proposed Focusing Technique using MUSIC-like Formulation 61

4.3 Recursive MUSIC-like Algorithms for Broadband Sources . . . . . . 66

4.3.1 Derivative Based Recursive MUSIC-like Algorithm . . . . . 66

4.3.2 Frequency Focusing Based Recursive MUSIC-like Algorithm 68

4.3.3 Bearing-Frequency Focusing Based Recursive MUSIC-like Algorithm .......................... 69

4.4 Simultaneous Orthogonal Matching Pursuit for Broadband Sources 71

4.5 Simulation Results . . . . . . . . . . . . . . . . . . . 75

4.5.1 Simulation Setup . . . . . . . . . . . . . 76

4.5.2 Performance Evaluation . . . . . . . . . . . . . 77

4.6 Conclusions ......................... 85

Chapter 5 Array Geometry Design for Narrow Band Compressive Sensing DOA Estimation 88

5.1 Introduction . . . . . . . . . . . . . . . . 88

5.2 The measurement Matrix . . . . . . . . . . . . . . . . . 91

5.3 Discussion Regarding Geometry . . . . . . . . . . . . . . . 93

5.4 Penrose Tiling . . . . . . . . . . . . . . . . . . . . . . . . 94

5.5 Danzer Tiling . . . . . . . . . . . . . . . . . 95 
5.6 Array Formation Using Inflation . . . . . . . . . . . . 96

5.6.1 The Rotation Matrix . . . . . . . . . . . . . . . 96

5.6.2 The Translation Matrix . . . . . . . . . . . . . . 97

5.6.3 The Scaling Matrix . . . . . . . . . . . . . . . . 97

5.6.4 The Flipping Matrix . . . . . . . . . . . . . . . . 97

5.6.5 Penrose Tiling Using Inflation Process . . . . . . . . . . . . 99

5.6.6 Danzer Tiling Using Inflation Process . . . . . . . . . . . . . 103

5.7 Optimization Scheme/Algorithm . . . . . . . . . . . 106

5.8 Boundary Constraints for the Optimization Problem . . . . . . . . 110

5.9 Results . . . . . . . . . . . . . . . . . . . 114

5.10 Conclusion . . . . . . . . . . . . . . . . . 126

Chapter 6 Array Geometry Design for Broadband Compressive $\begin{array}{ll}\text { Sensing DOA Estimation } & 128\end{array}$

6.1 Introduction . . . . . . . . . . . . . . . . . . . . 128

6.2 Geometry Optimization Problem for Broadband DOA Estimation . 129

6.3 Boundary Constraints for Optimization Problem . . . . . . . . . . . 132

6.4 Optimization Results for Aperiodic Geometries . . . . . . . . . . . 135

6.4.1 Simulation Setup . . . . . . . . . . . 135

6.4.2 Resultant Geometries . . . . . . . . . . . . . . . 141

6.5 Performance Results . . . . . . . . . . . . . . . . . . . . . 143

6.6 Conclusions . . . . . . . . . . . . . . . . . . . 154

$\begin{array}{lll}\text { Chapter } 7 & \text { Conclusions and Future Works } & 155\end{array}$

7.1 Conclusions . . . . . . . . . . . . . . . . . . 155

7.2 Future Works . . . . . . . . . . . . . . . . . . . 158 
Appendix A Coordinate System

Appendix B Complex Orthogonal Procrustes Problem

Bibliography 


\section{Summary}

Array Signal Processing has multitude of applications. With the advent of new applications like self-driving cars and $5 \mathrm{G}$ mobile communications, this field has seen a renewal in interest from researchers. Compressive sensing, on the other hand is a comparatively new field of research and has generated tremendous interest in the past decade, since its conception. In this thesis, we explore integration of Compressive Sensing (CS) algorithms with the problem of Direction of Arrival (DOA) estimation through an array of antennas/sensors. Compressive Sensing has gained popularity in multitude of fields due to its ability of successful recovery of subNyquist sampled signals, which was previously thought of as unlikely, if not all out impossible. The main goal of this thesis is to achieve efficiency and reduction of resources (e.g. number of array elements) through the use of CS algorithms to the problem of DOA estimation using antenna arrays. Antenna arrays used in compressive sensing based algorithms are generated randomly to minimize mutual coherence, a property of sensing matrix (which is also referred to as "array manifold matrix" in array processing jargon). Random sampling of aperture, although useful for compressive sensing, suffers from practical limitations. For an antenna array that is sufficiently random, some elements of the array would almost always fall very close to each other, which is infeasible to implement in a practi- 
cal scenario. Rectangular arrays, although very uniform, suffer from a very high mutual coherence, which in turn degrades the performance of CS algorithms. Aperiodic arrays are a compromise solution. We demonstrate, in this thesis, that it is possible to design aperiodic arrays that perform much better than rectangular arrays by using a simple disturbance optimization scheme, which can be applied to other aperiodic geometries as well. We use Danzer tiling as a base geometry and device an optimization scheme, which uses very few parameters to generate an aperiodic geometry. We extend this framework to design aperiodic array geometries for broadband DOA estimation scenario and apply this technique to array geometries formed based on Penrose and Danzer aperiodic tiling. Furthermore, we study the problem of estimating DOA when we have multiple measurement vectors (MMV). The classical approach to address this problem is to use the MUSIC algorithm. But to use this algorithm, we must have complete knowledge of the number of sources. This drawback was eliminated by a MUSIC-like algorithm that does not presuppose the knowledge of the number of sources. This algorithm, computes the covariance matrix from MMV (or snapshots of data.) The covariance matrix has to be full rank for the algorithm to work properly. We, in thesis, devise a recursive MUCIC-like algorithm based on a generalized MUSIC criterion, which is capable of estimating DOA even when the covariance matrix is rank deficient. This algorithm is based on the principle of greed, which treats local maximum at every step as a global maximum. We compare this algorithm with other greedy algorithms like Simultaneous Orthogonal Matching Pursuit (SOMP) algorithm and show that its performance is better than SOMP. Lastly, we address the problem of estimating DOA for broadband sources using Compressive Sensing algorithms. The recursive algorithm, discussed previously, is extended by using Taylor series expansion and focusing matrices approach to estimate the broadband sources. We also extend well 
known MMV algorithm, SOMP, to estimate DOA of broadband sources. 


\section{List of Figures}

3.1 Comparison of DOA estimation capabilities of the two formulations of MUSIC-like algorithm for $\mathrm{r}=2, \mathrm{M}=31, \mathrm{~K}=4$ and $\mathrm{SNR}=40$ $\mathrm{dB}$. The four vertical lines denote the locations of the actual sources located at 55, 78, 90 and 120 degrees. . . . . . . . . . . 34

3.2 Pseudo-spectrum for generalized MUSIC criterion (2.50) and generalized MUSIC-like criterion (3.14) when $\mathrm{M}=31, \mathrm{~K}=4, \mathrm{r}=2$ and $\mathrm{SNR}=20 \mathrm{~dB} \ldots \ldots \ldots \ldots \ldots$

3.3 Comparison of generalized MUSIC criterion with generalized MUSIClike criterion when the estimate of dimension of $\boldsymbol{Q}$ is wrong. . . .

3.4 Stopping function $\eta(J)$ shown for $\mathrm{M}=31, \mathrm{~K}=6$ and $\mathrm{SNR}=0 \mathrm{~dB}$, when the covariance matrix is a) full rank $(\mathrm{r}=100)$, b) rank deficient $(\mathrm{r}=4) \ldots \ldots \ldots \ldots \ldots \ldots \ldots$

3.5 Recovery rate maps for different values of $\mathrm{M}$ and $\mathrm{K}$ when $\mathrm{SNR}=20$ $\mathrm{dB}$ and $\mathrm{N}=180$, the white line defines the boundary between the recovery region and the non-recovery region, a) Recovery rate map for SOMP when $\mathrm{r}=4$, b) Recovery rate map for SOMP when $\mathrm{r}=$ 16, c) Recovery rate map for RML when $\mathrm{r}=4$, d) Recovery rate map for RML when $\mathrm{r}=16 . \ldots \ldots \ldots$

3.6 $P_{R}$ vs SNR curves for RML and SOMP algorithm for values of $\mathrm{M}=$ 39 and $\mathrm{K}=9 . \ldots \ldots \ldots \ldots$ 
3.7 MSE for two sources plotted against SNR for RML and SOMP for $r=10, M=11$ and a) a source at 37 degrees, b) a source at 43

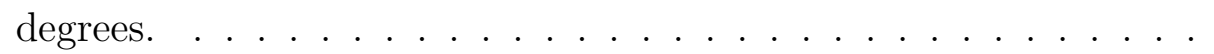

3.8 MSE for two sources plotted against SNR for RML and SOMP for $r=100, M=11$ and a) a source at 35 degrees, b) a source at 39 degrees. Note that the horizontal axis has been shifted left for this figure as compared to Figure 3.7 as there are more snapshots available. 51

4.1 10-element circular array used for broadband sources DOA estimation using MUSIC-like (focusing scheme) and Robust auto-focusing scheme (MUSIC). The inner circle has a radius of $3 \lambda / 2$ while the outer circle has a radius of $4 \lambda / 2 \ldots \ldots \ldots$. . . . . . . . . .

4.2 Pseudo-Spectrum comparison for uniform linear geometry for MUSIClike (focusing scheme) and Robust auto-focusing scheme (MUSIC), a) SNR value of $0 \mathrm{~dB}$ and b) SNR value of $20 \mathrm{~dB}$. . . . . . . .

4.3 Pseudo-Spectrum comparison for circular geometry for MUSIC-like (focusing scheme) and Robust auto-focusing scheme (MUSIC) a) SNR value of $0 \mathrm{~dB}$ and b) SNR value of $20 \mathrm{~dB} . \ldots . . . .$.

4.4 Comparison of Narrowband MUSIC algorithm with the Robust autofocusing algorithm (Section 4.2.2) for two sources located at 65 and 75 degrees respectively. $\mathrm{SNR}$ is $10 \mathrm{~dB}$, the number of sensors $\mathrm{M}=$ 20 in uniform linear array configuration. Two frequency bins have been used, while, the source signal normalized bandwidth 0.1. The vertical lines indicate the direction of the two sources. . . . . . . . 
4.5 Two iterations of Frequency focusing based SOMP algorithm. The two dotted lines show the actual direction of the two sources at 65 and 75 degrees respectively. This simulation was performed for signal with 10-dB SNR, the number of sensors were $M=20$ in uniform linear array configuration. Two frequency bins have been used, while, the source signal normalized bandwidth 0.1 . . . . . . . . . . . . . 74

4.6 Phase transition plots for the four algorithms for $\mathrm{r}=2, \mathrm{D}=16$ and $\mathrm{SNR}=40 \mathrm{~dB} . \ldots \ldots \ldots \ldots \ldots$

4.7 Phase transition plots for the four algorithms for $\mathrm{r}=2, \mathrm{D}=32$ and $\mathrm{SNR}=40 \mathrm{~dB} . \ldots \ldots \ldots \ldots \ldots \ldots$

4.8 Phase transition plots for the four algorithms for $\mathrm{r}=2, \mathrm{D}=128$ and $\mathrm{SNR}=40 \mathrm{~dB} \ldots \ldots \ldots \ldots \ldots \ldots$

4.9 Phase transition plots for the four algorithms for $\mathrm{r}=2, \mathrm{D}=256$ and $\mathrm{SNR}=40 \mathrm{~dB}$.

4.10 Phase transition plots for the four algorithms for $\mathrm{r}=16, \mathrm{D}=16$ and $\mathrm{SNR}=40 \mathrm{~dB}$.

4.11 Phase transition plots for the four algorithms for $\mathrm{r}=16, \mathrm{D}=32$ and $\mathrm{SNR}=40 \mathrm{~dB}$.

4.12 Phase transition plots for the four algorithms for $\mathrm{r}=16, \mathrm{D}=128$ and $\mathrm{SNR}=40 \mathrm{~dB} \ldots \ldots \ldots \ldots$

4.13 Phase transition plots for the four algorithms for $\mathrm{r}=16, \mathrm{D}=256$ and $\mathrm{SNR}=40 \mathrm{~dB} \ldots \ldots \ldots 82$

$4.14 P_{R}$ vs SNR plots for $\mathrm{r}=2$ and $\mathrm{D}=32$ and $\mathrm{M}=31 \ldots . . . . \quad 83$

4.15 $P_{R}$ vs SNR plots for $\mathrm{r}=100$ and $\mathrm{D}=256$ and $\mathrm{M}=10$. Please note that the horizontal axis is shifted left as compared to Figure 4.14 as there are more snapshots and thus the performance is better. . . . 
4.16 MSE plot for $\mathrm{r}=2$ and $\mathrm{D}=32$ and $\mathrm{M}=31$ a) Source 1 at 78 degrees, b) Source 2 at 94 degrees, c) Source 3 at 110 degrees. Note that the vertical axis is not constant for the three sub-figures.

4.17 MSE plot for $\mathrm{r}=100$ and $\mathrm{D}=256$ and $\mathrm{M}=10$ a) Source 1 at 78 degrees, b) Source 2 at 94 degrees, c) Source 3 at 110 degrees. Since the number of snapshots are much larger for this figure as compared to Figure 4.16, the horizontal axis is shifted as the detection is better even at poor SNR. Also note the vertical scale is not constant for the three sub-figures. . . . . . . . . . . . . . . .

5.1 Basic Penrose Tiles. . . . . . . . . . . . . . . . . . . . . . . 94

5.2 Basic Danzer Tiles. . . . . . . . . . . . . . . . . . . . . 95

5.3 Basic Penrose Tiles. . . . . . . . . . . . . . . . . . . . . 100

5.4 Level I Penrose Tiles. . . . . . . . . . . . . . . . . . . . . . 100

5.5 Level III Penrose Tile AL. . . . . . . . . . . . . . . . . . . . . . 101

5.6 Level I Danzer Tiles. . . . . . . . . . . . . . . . . . . . . . . . . . 104

5.7 a) Level-3 Danzer aperiodic tiling b) Sensors array antenna formed by placing sensors at the indices of each triangle. . . . . . . . . 107

5.8 Optimization constraints depicted by lines for a) Danzer Tile 1, b) Danzer Tile 2, c) Danzer Tile 3. . . . . . . . . . . . . . . . . . . 111

5.9 Optimization constraints depicted by lines for a) Penrose Tile AL, b) Penrose Tile AS. . . . . . . . . . . . . . . . . . . . . . . . . 112

5.10 a) 48-element random array, b) 48-element constrained random array, c) 94-element triangular array (rectangular grid sampling), d) 48element Danzer array, e) 48-element optimized Danzer array. . . . . 115 
5.11 Correlation matrices for different geometries using Danzer tile as aperture. High energy in off-diagonal elements indicate high mutual coherence. . . . . . . . . . . . . . . . 118

5.12 a) 55-element random array, b) 55-element constrained random array, d) 55-element Penrose array, e) 55-element optimized Penrose array. 119

5.13 Correlation matrices for different geometries using Penrose tile as aperture. High energy in off-diagonal elements indicate high mutual coherence. ..................... . . 121

5.14 Mean Squared Error for different SNR values plotted for different geometries shown in Figure 5.10 based on Danzer tile-II aperture. . 125

5.15 Mean Squared Error for different SNR values plotted for different geometries shown in Figure 5.12 based on Penrose tile- $A L$ aperture

6.1 Optimization constraints depicted by lines for a) Danzer Tile 1, b) Danzer Tile 2, c) Danzer Tile 3. . . . . . . . . . . . . . . . . 133

6.2 Optimization constraints depicted by lines for a) Penrose Tile AL, b) Penrose Tile AS. . . . . . . . . . . . . . . . . . . . 134

6.3 Level IV Penrose Tile AL (Danzer Geometry 1). . . . . . . . . . . . 135

6.4 Level II Danzer Tile 1 (Danzer Geometry 2) . . . . . . . . . . . . . 135

6.5 Level II Danzer Tile 2 (Penrose Geometry 1). . . . . . . . . . . . . 136

6.6 Optimal points for Danzer Geometry 1 a) Danzer Tile 1, b) Danzer Tile 2, c) Danzer Tile 3. . . . . . . . . . . . . . . . . . . . 138

6.7 Optimal points for Danzer Geometry 2 geometry a) Danzer Tile 1, b) Danzer Tile 2, c) Danzer Tile 3. . . . . . . . . . . . . . . . . 139

6.8 Optimal points for Penrose Geometry 1 a) Penrose Tile AL, b) Penrose Tile AS. . . . . . . . . . . . . . . . . . . . . . 140 
6.9 Danzer Tile 1 aperture a) 48-element random array, b) 48-element constrained random array, c) 60-element triangular array (rectangular grid sampling), d) 48-element Danzer array, e) 48-element optimized aperiodic array. . . . . . . . . . . . . . . . . . . . 142

6.10 Danzer Tile 2 aperture a) 87-element random array, b) 87-element constrained random array, c) 111-element triangular array (rectangular grid sampling), d) 87-element Danzer array, e) 87-element optimized aperiodic array. . . . . . . . . . . . . . . . 144

6.11 Penrose Tile AL aperture a) 45-element random array, b) 45-element constrained random array, c) 48-element uniform sampling array, d) 45-element Danzer array, e) 45-element optimized aperiodic array.

6.12 SNR vs the probability of resolution $\left(P_{R}\right)$ for Danzer Tile 1 aperture. 148

6.13 SNR vs the probability of resolution $\left(P_{R}\right)$ for Danzer Tile 2 aperture. 148

6.14 SNR vs the probability of resolution $\left(P_{R}\right)$ for Penrose Tile AL aperture.149

6.15 MSE plot for geometries based on Danzer Tile 1 aperture a) Target 1 at 60 degrees azimuth, b) Target 2 at 94 degrees azimuth, c) Target 3 at 144 degrees azimuth. Note that the vertical axis is not constant for the sub-figures. . . . . . . . . . . . . . . . . . . . 151

6.16 MSE plot for geometries based on Danzer Tile 2 aperture a) Target 1 at 78 degrees azimuth, b) Target 2 at 94 degrees azimuth, c) Target 3 at 114 degrees azimuth. Note that the vertical axis is not constant for the sub-figures. . . . . . . . . . . . . . . . . 152

6.17 MSE plot for geometries based on Penrose Tile AL aperture a) Target 1 at 78 degrees azimuth, b) Target 2 at 94 degrees azimuth, c) Target 3 at 114 degrees azimuth. Note that the vertical axis is not constant for the sub-figures. . . . . . . . . . . . . . . . 153 
A.1 The Coordinate System. $\theta$ is the azimuth angle, while $\phi$ is the polar angle. . . . . . . . . . . . . . . . . 161 


\section{List of Tables}

4.1 Parameters used for comparison of the two schemes (MUSIC-like focusing scheme and MUSIC Robust auto-focusing scheme ) . . . . 63

4.2 Parameters used for comparison of the broadband RML algorithms $\quad 77$

5.1 Geometrical properties of different antenna array designs using Danzer array geometry (Figure 5.10 ). The minimum nearest neighbor distance(5.75). The average nearest neighbor distance(5.77). The maximum nearest neighbor distance(5.76) . . . . . . . . . . . . . . 123

5.2 Geometrical properties of different antenna array designs using Penrose array geometry (Figure 5.12). The minimum nearest neighbor distance (5.75). The average nearest neighbor distance (5.77). The maximum nearest neighbor distance (5.76) . . . . . . . . . . . . 124

6.1 Parameters used for the optimization problem in (6.15) . . . . . . 137

6.2 Parameters for simulation . . . . . . . . . . . . . 146 


\section{List of Acronyms}

CS Compressive Sensing / Compressed Sensing

RADAR Radio Detection and Ranging

SONAR Sound Navigation and Ranging

SAR Synthetic Aperture Radar

FMCW Frequency Modulated Continuous Wave

MUSIC Multiple Signal Classification

OMP Orthogonal Matching Pursuit

SOMP Simultaneous Orthogonal Matching Pursuit

BP Basis Pursuit

BPDN Basis Pursuit De-noising

RIP Restricted Isometry Property

SMV Single Measurement Vector

MMV Multiple Measurement Vectors

ML MUSIC-like

RML Recursive MUSIC-like

ULA Uniform Linear Array

ESPRIT Estimation of Signal Parameters via Rotational Invariance Technique

SA-MUSIC Subspace Augmented MUSIC

GMC Generalized MUSIC Criterion

GMLC Generalized MUSIC-like Criterion

SNR Signal-to-Noise Ratio

TSE Taylor Series Expansion 


\section{List of Symbols}

\section{Chapter 2}

\begin{tabular}{|c|c|}
\hline$c$ & Speed of light in vacuum \\
\hline$\epsilon_{0}$ & Electrical constant or vacuum permittivity \\
\hline$\mu_{0}$ & Magnetic constant or vacuum permeability \\
\hline B & Magnetic field vector \\
\hline $\mathbf{J}$ & Current density vector \\
\hline$\nabla \cdot$ & Divergence of a vector field \\
\hline$\nabla \times$ & Curl of a vector field \\
\hline$x(t, \boldsymbol{\rho})$ & Amplitude of the wave at location $\boldsymbol{\rho}$ and time $\mathrm{t}$ \\
\hline$\rho$ & Location in $3 \mathrm{D}$ space \\
\hline$\kappa$ & Wavenumber vector \\
\hline$\kappa$ & Magnitude of the wavenumber vector \\
\hline$f$ & Frequency of the wave \\
\hline$\omega$ & Angular frequency of the wave \\
\hline$\alpha$ & Amplitude of the received signal \\
\hline$t$ & Time \\
\hline$\lambda$ & Wavelength of the wave \\
\hline$K$ & Number of sources \\
\hline$y_{m}(t)$ & Received signal by sensor $m$ at time $t$ \\
\hline $\boldsymbol{y}(t)$ & Received signal vector at time $t$ \\
\hline$M$ & Number of sensors \\
\hline$d$ & Separation between two sensors in ULA configuration \\
\hline$\theta$ & Angle of arrival \\
\hline$\theta_{k}$ & Angle of arrival of source $k$ \\
\hline $\boldsymbol{a}\left(\theta_{k}\right)$ & Steering vector for continuous direction $\theta_{k}$ \\
\hline $\boldsymbol{A}(\Theta)$ & Array manifold matrix \\
\hline
\end{tabular}




\begin{tabular}{|c|c|}
\hline $\boldsymbol{a}(j)$ & Steering vector for discrete direction index $j$ \\
\hline $\boldsymbol{\eta}(t)$ & Noise vector at time $t$ \\
\hline $\boldsymbol{H}()$ & Noise matrix \\
\hline$\mu$ & Mean of the noise vector $\boldsymbol{\eta}(t)$ \\
\hline$\Sigma$ & Covariance matrix \\
\hline$T_{s}$ & Sampling time period \\
\hline$F_{s}$ & Sampling frequency \\
\hline$r$ & Number of snapshots or MMV \\
\hline$\Gamma_{d n s}$ & Spatial spectrum of delay-and-sum beamformer \\
\hline$\Gamma_{m v d r}$ & Spatial spectrum of MVDR beamformer \\
\hline$\Gamma_{\text {music }}$ & Spatial spectrum of MUSIC algorithm \\
\hline $\boldsymbol{a}\left(f_{i}, \theta\right)$ & Frequency dependent broadband steering vector \\
\hline $\boldsymbol{A}(f, \Theta)$ & Broadband array manifold matrix \\
\hline$\Gamma_{I S S M}$ & Spatial spectrum of ISSM algorithm \\
\hline$\Gamma_{I S S M-G}$ & Spatial spectrum of geometric mean based ISSM algorithm \\
\hline $\boldsymbol{z}(i)$ & Focused output of array for frequency $f_{i}$ \\
\hline $\boldsymbol{T}\left(f_{i}, \hat{\Theta}\right)$ & Frequency focusing transformation matrix \\
\hline$\Psi$ & Basis matrix \\
\hline$\Phi$ & Sensing matrix \\
\hline$P_{(.)}$ & Subspace projection operator \\
\hline
\end{tabular}




\section{Chapter 3}

N Total number of directions

$\boldsymbol{Y} \quad$ Matrix containing multiple measurement vectors

$\boldsymbol{X} \quad$ Sparse source matrix

$\boldsymbol{A} \quad$ Extended array manifold matrix

$\boldsymbol{l}_{j} \quad 3 \mathrm{D}$ position vector of $j^{\text {th }}$ sensor

$\boldsymbol{L} \quad$ Sensor location matrix

$\boldsymbol{H} \quad$ Noise matrix

$\Gamma_{\text {music-like }}$ Pseudo-spectrum of MUSIC-like algorithm

$\beta \quad$ Optimization parameter of MUSIC-like algorithm

$\boldsymbol{w}_{i} \quad$ Weight vector solution for direction index $i$

$\boldsymbol{W} \quad$ Matrix containing weight vectors from $\mathrm{N}$ directions

$\boldsymbol{S}_{J} \quad$ Vector containing estimated source indices

$\boldsymbol{A}_{\boldsymbol{S}_{J}} \quad$ Array manifold matrix containing steering vectors from directions $\boldsymbol{S}_{J}$

$\eta[J] \quad$ Stoppage criterion

$\Sigma_{y y} \quad$ Real output covariance matrix

$\Sigma_{s s} \quad$ Source covariance matrix

$\boldsymbol{R} \quad$ Computer output covariance matrix

$\sigma_{v}^{2} \quad$ Noise variance 


\section{Chapter 4}

$\boldsymbol{v}_{\theta_{s}} \quad$ Unit vector in the direction of the source

$J \quad$ Derivative order

$D \quad$ Number of frequency bins

$\boldsymbol{A}(f, \theta) \quad$ Array manifold matrix for broadband signals

$\boldsymbol{a}(f, \theta) \quad$ Steering vector for broadband signals

$\boldsymbol{D}_{f}(\theta) \quad$ Derivative manifold matrix

$\boldsymbol{z}\left(f_{i}\right) \quad$ Focused output for frequency $f_{i}$

$\boldsymbol{T}(i) \quad$ Focusing transformation matrix for frequency bin $i$

\section{Chapter 5}

$\boldsymbol{R}(\theta) \quad$ Rotation matrix

$\boldsymbol{T}(x, y) \quad$ Translation matrix

$\boldsymbol{S}(s x, s y) \quad$ Scaling matrix

$\boldsymbol{p}_{i} \quad$ Position vector for sensor $i$

$\boldsymbol{T} \quad$ Transformation matrix

$\boldsymbol{B} \quad$ Mutual coherence matrix defined as $\boldsymbol{A}^{H} \boldsymbol{A}$

$M_{u} \quad$ Slope matrix for boundary lines

$\boldsymbol{C}_{u} \quad$ y-intercept matrix for boundary lines 


\section{Chapter 6}

$\boldsymbol{A}(l, m, n) \quad$ Broadband array manifold matrix

$L \quad$ Number of frequency bins

$\boldsymbol{B}_{l} \quad$ Mutual coherence matrix for frequency bin $l$ 


\section{Chapter 1}

\section{Introduction}

\subsection{Background}

A system consisting of multiple sensors/transducers (antennas, microphones, seismic sensors, etc.) employed to capture signals generated from a single or multiple sources of interest is dealt with by the theory of Array Signal Processing. Array Signal Processing has a lot of important applications and is an active area of research. There exists a wide array of literature dealing with the concepts of this important sub-field of Signal Processing. Some of the literature dealing with the study of array signal processing is provided in the bibliography $[1,2,3]$.

Following are some of the important applications of Array Signal Processing.

i Communication: Wireless communication has become a very important part of the modern life with the advent of mobile communications. The bandwidth-hungry applications of the modern Internet have revived interest in array processing for the modern 5G mobile communication [4]. 
ii Radar and Sonar: Radars have been in use since 1940s mainly for military applications. The improvement in digital technologies and lowering costs of radar technologies have made the use of radar in other areas more appealing. Now radars have found uses in self driving cars, sports technology equipment and medical equipment, besides plethora of other non-military applications. With the microwave transceiver technologies such as Frequency Modulated Continuous Wave (FMCW) radars getting compact and cheap and Unmanned Air Vehicles (UAV) becoming readily available, Synthetic Aperture Radar (SAR) has also gained interest. It can be used for high resolution imaging regardless of the weather conditions and time of the day, unlike the camera based imaging techniques that suffer severely under these hostile conditions. Sonar is used for under-water target localization and this technology has multiple other applications as well, besides military applications, the primary purpose of its invention.

iii Seismology: Seismic survey for oil and gas exploration and geology study uses array processing techniques at its core. The sensor array detects rebounding vibrations from layers of rocks beneath the earth surface when a charge is exploded to generate a transmit signal. The received signal from using an array of sensors is then analyzed to extract information regarding geological properties of the rock layers.

iv Speech Processing: Microphone arrays are used for DOA estimation and blind source separation and denoising applications.

All these applications make use of an array of sensors/transducers. The signal is received through these transducers and converted to voltage signal in the receiver 
and finally converted into a digital signal using analog to digital converters (ADCs). This digital signal forms a vector at each time instant. The vector signal is then processed to obtain some additional information about the source, that would not be possible with the use of a single scalar sensor.

One important parameter is source signal detection. Detection is the process of identifying/separating the source signal from background noise or clutter. For radar applications, reliable detection of signal is very important to avoid false alarm or false positive. The detection process doesn't require multiple sensors and thus isn't exclusive to the domain of array signal processing. But it is an important first step to start estimation of the other important parameters.

One important parameter is the Direction of Arrival (DOA) of the signal. The signal originating (or reflecting) from a source (or target) travels in a medium with some known velocity and because of the separation between the sensors, the signal arrives at each sensor at a different time. This difference in arrival time is then used to estimate the direction of the signal origin. The precision of the estimation of DOA of the signal is an active area of research and this thesis is also related to this field. If the signal is very strong as compared to the electronics noise or interference signal, then the direction of the signal can be estimated to a reasonable degree of accuracy using conventional techniques like delay-and-sum beamforming.

\subsection{Objectives and Motivation}

The problem arises when the signal is very weak and is buried in noise. For such a scenario, the usual techniques fail to produce reliable results. Some of the state of the art techniques like the Multiple Signal Classification (MUSIC) algorithm [29] 
is used in this scenario. This algorithm requires a prior knowledge of the number of sources for accurate estimation of the DOA. Akaike Information Criterion (AIC) [6] or Minimum Description Length (MDL)[7] are used to estimate the model order or the number of sources present to provide the initial conditions for the MUSIC algorithm. But in the presence of noise, these techniques can lead to erroneous model order estimation. A more robust method called the "MUSIC-like algorithm" was recently proposed [28], which eliminated the need for model order estimation and still has a good performance.

Compressive sensing [9] is a set of techniques that has found a lot of interest from engineering/mathematics community in the previous decade since its original formulation, almost a decade ago. It deals with the exact recovery of the severely undersampled signals, if the signals have sparse representation in some domain and the sampling domain is incoherent with that particular domain. In the case of direction of arrival estimation, if there are a very few number of sources, then the representation of these sources in the domain representing all the possible directions is already sparse. The sensing domain, as would become apparent in the next chapter, is Fourier domain and is incoherent with the signal representation domain. So it is possible, according to the theory of Compressive sensing, to use very few time samples, or under-sample the signal by using very few sensors (spatial-domain undersampling), and still obtain a reasonable estimate of direction of arrival of the signal.

The techniques already available to achieve this goal are lacking in several aspects. For instance, the famous $l_{1}$ recovery algorithm called basis pursuit de-noising [12] algorithm doesn't perform well, when the signal is very weak as compared to the noise or when the SNR is low. If we have multiple measurement vectors, as is needed to form a covariance matrix for the MUSIC algorithm, the CS algorithms' 
performance is not as good as compared to the performance of the MUSIC algorithm. The measurement vector integration capabilities of the MUSIC algorithm are superior to any of the existing CS algorithms. Our objective is to form an algorithm that has integration capabilities of the MUSIC algorithm but still has the advantages of CS algorithms, where the MUSIC algorithm fails.

Sensor array geometry plays an important role in the application of CS algorithms to DOA estimation. The geometry and the placement of the sensors is an important factor in deciding the recovery capabilities of the CS algorithms. The ideal configuration for sensor placement is a random configuration, which minimizes the mutual coherence between the sensing domain and the signal representation domain, thus improving the probability of estimating the DOA from a very few sensors than the classical techniques entail. This configuration, is however impractical as some sensors would almost always fall too close to each other for them to be fabricated on a circuit board or mounted in physical space. This serves as a motivation to find a geometry that is sparse than the usual geometries employed but is still able to estimate DOA as much reliably as a random configuration based geometry.

The above discussion is valid when the signal is spread in a narrow band of frequencies that can be approximated as a single frequency. For the case, when a wideband signal that is spread over a broad band of frequencies, specialized techniques are needed. Focusing matrices and Taylor Series approximation based techniques are employed to address the problem of DOA estimation of the wideband signals. The model order estimation suffers from the same limitations as discussed previously for the case of the narrowband estimation. No CS algorithm exists to solve this problem. Our objective is to formulate CS algorithms for broadband case, that uses multiple measurement vectors, combines them efficiently and reduces the number of sensors used. 
Broadband signals pose a problem when it comes to the the geometry of the sensor array. The regular geometries or the uniform geometries are not well suited for wideband applications, as they introduce grating lobes, thus injecting ambiguities in estimation of DOA. This gives us motivation to explore the use of irregular geometries, that are well suited to the broadband applications as well as sparse enough, so can be used with CS algorithms.

\subsection{Major Contributions of the Thesis}

The author testifies to the originality of the work presented in this thesis. The following are the original contributions of the author in this regard:

i Devising a mathematical framework by deriving bounds for the $\beta$ parameter that do not depend on covariance matrix inversion. For MUSIC-like algorithm to be used in Compressive sensing scenario, any dependence of the bounds on matrix inversion is counter-productive as the inverse would not exist for a rank deficient matrix. Here CS implies the use of a very few snapshots or MMV, as a consequence of which, the correlation matrix becomes degenerate. Deriving a recursive formulation of the algorithm, as the MUSIC-like algorithm in the original form is not suitable to be used with degenerate covariance matrix. The main advantage is that even with very few snapshots, in which case both MUSIC and MUSIC-like algorithms fail, the Recursive Music-like (RML) algorithm can still be used to estimate DOA of unknown number of sources. Formulation of a stoppage criterion for the recursive algorithm based on projection matrices of the estimated source indices. This work has been published [C1]. 
ii Extension of narrowband RML algorithm, derived in Chapter 3, to broadband sources. In the process, four new recursive algorithms were devised based on the RML principle. These four algorithms can be used with degenerate covariance matrix for broadband sources, where conventional broadband algorithms (like Coherent Signal Subspace Method (CSSM)) fail. Formulation of a new broadband algorithm for DOA estimation of unknown number of broadband sources using simultaneous bearing-frequency focusing. This work is under publication [J1].

iii Formulation of a perturbation-based optimization technique to generate sparse aperiodic geometry for use with CS based DOA algorithms. The geometry is based on aperiod tiling such as Danzer-tiling and Penrose-tiling. Deriving of mathematical equations and modeling inflation based array geometry design and formulating an optimization problem to minimize the mutual coherence between the sensing domain and the representation domain of the signal. This work has been submitted for publication [J2].

iv The mathematical formulation of the optimization problem for aperiodic geometry realization for DOA estimation of broadband sources using an algorithm developed by the author in Chapter 4. Realization of an optimization problem that takes care of the frequency-dependence of the steering vector for the broadband sources. This work has been submitted for publication [J3].

\subsection{Organization of the Thesis}

The thesis is organized into six more chapters. Chapter 2 provides the review of concepts of the material related to the thesis. In Chapter 3, we propose a Recursive 
MUSIC-like (RML) algorithm for estimation of DOA of unknown number of sources for very few measurement vectors and very few sensors. Chapter 4 details extension of the RML algorithm to broadband signals and several algorithms are developed based on different methodologies. In Chapter 5, we develop an optimization scheme for geometry based on aperiodic tiling for a CS based algorithm: SOMP. Chapter 6 details the design of optimal geometry for broadband signals using one of the algorithms developed in the preceding chapter. Chapter 7 concludes the preceding discussion and elaborates on the future extensions of the work undertaken in this thesis. 


\section{Chapter 2}

\section{Review of Concepts}

In this chapter, we review some of the concepts related to array signal processing and compressive sensing. The review is brief but exhaustive enough not to omit anything that is necessary in understanding the rest of the chapters in this thesis. The applications discussed in the previous chapter mainly uses radio-waves or microwaves. These waves are governed by principles derived in Maxwell's theory of electromagnetism. Maxwell also derived the wave equation for propagation of the electromagnetic waves in a medium. We will use the general from of this equation to derive the steering vector and array manifold matrix later in this chapter.

\subsection{Maxwell's Theory of Electromagnetism}

For electromagnetic propagation, Maxwell's field theory [5] gives us a good insight into the working principles of the phenomenon. Due to changing electrical and magnetic fields, we get an electromagnetic wave, that travels with a constant speed in a medium. The speed $(c)$ in vacuum is given by the following equation: 


$$
c=\frac{1}{\sqrt{\mu_{0} \epsilon_{0}}} .
$$

Here, $\epsilon_{0}$ is the electrical constant or vacuum permittivity, while $\mu_{0}$ is magnetic constant or vacuum permeability. These constants arise from the Gauss's law and Ampere's law, respectively.

Ampere's circuital law [38] is given by the following equation:

$$
\oint_{C} \mathbf{B} \cdot \mathrm{d} \boldsymbol{\ell}=\mu_{0} \iint_{S} \mathbf{J} \cdot \mathrm{d} \mathbf{S}=\mu_{0} I_{\mathrm{enc}}
$$

$\mathbf{B}$ is the magnetic field, $\mathbf{J}$ is the current density. The same law can be written in the following manner as a differential equation:

$$
\nabla \times \mathbf{B}=\mu_{0} \mathbf{J}
$$

The law that governs electrostatic forces is called Gauss's law or Gauss's flux theorem. It is given by the following integral equation:

$$
\mathbf{E} \cdot \mathrm{d} \mathbf{S}=\frac{1}{\varepsilon_{0}} \iiint_{\Omega} \rho \mathrm{d} V
$$

The differential form is given as follows:

$$
\nabla \cdot \mathbf{E}=\frac{\rho}{\varepsilon_{0}}
$$

$\nabla \cdot$ is the divergence of any vector field, $\mathbf{F}=U \mathbf{i}+V \mathbf{j}+W \mathbf{k}$, and is given by

$$
\operatorname{div} \mathbf{F}=\nabla \cdot \mathbf{F}=\left(\frac{\partial}{\partial x}, \frac{\partial}{\partial y}, \frac{\partial}{\partial z}\right) \cdot(F x, F y, F z)=\frac{\partial U}{\partial x}+\frac{\partial V}{\partial y}+\frac{\partial W}{\partial z}
$$

Maxwell combined the electrical and magnetic laws as shown above by giving a complete theory of electromagnetism. He corrected the Ampere's law by adding an additional term to the original Ampere's law:

$$
\nabla \times \mathbf{B}=\mu_{0}\left(\mathbf{J}+\varepsilon_{0} \frac{\partial \mathbf{E}}{\partial t}\right)
$$


This new term shows that not only the changing magnetic field induce an electric field, but also a changing electrical field induces a magnetic field. These equations allow self sustaining electromagnetic waves to travel through empty space.

Solving the Maxwell's equations in the regions with no charges and no currents, leads to the following wave equations:

$$
\begin{aligned}
& \frac{1}{c^{2}} \frac{\partial^{2} \mathbf{E}}{\partial t^{2}}-\nabla^{2} \mathbf{E}=0 \\
& \frac{1}{c^{2}} \frac{\partial^{2} \mathbf{B}}{\partial t^{2}}-\nabla^{2} \mathbf{B}=0 .
\end{aligned}
$$

These equations govern the propagation of electromagnetic waves in space and time.

The disturbance in electrical and magnetic fields, and wave fronts travel at the speed of light, c. Wavefronts are the locus of points characterized by propagation of position of the same phase.

In this section, we have discussed mainly the theory of electromagnetism to understand the wave propagation. The concepts of array processing, however, are not limited only to electromagnetic waves. These concepts can be applied to other types of wave propagation. One such example is of mechanical waves, specifically sound waves. Sound waves are used to locate sources inside water, as the attenuation of electromagnetic waves is too great in this medium.

\subsection{Array and Steering Vector}

The solution to the second order homogeneous wave propagation equation (2.9) at time $t$ and spatial location $\boldsymbol{\rho}$ is the following exponential:

$$
x(t, \boldsymbol{\rho})=\alpha e^{j(\omega t-\kappa \cdot \rho)},
$$


where, $\omega$ is the angular frequency given by $\omega=2 \pi f . \quad f$ is the frequency of the electromagnetic wave. $\boldsymbol{\kappa}$ is called the wavenumber vector. It is given by $\boldsymbol{\kappa}=\frac{2 \pi}{\lambda} \boldsymbol{v}$. $\boldsymbol{v}$ is a unit vector that represents the direction of wave propagation. The amplitude of the signal is represented by $\alpha$.

The direction information of a signal source transmitting electromagnetic signal can be estimated by using an array of sensors. Each sensor/transducer constituting the array, samples the signal and converts it to a digital signal. Depending on the direction of the signal and the spacing between adjacent sensors, there is a certain delay at which the signal wavefront arrives at each sensor. The delay depends on the direction of the source signal.

In the case of a uniformly spaced linear array of sensors, the delay is given by

$$
\delta t=(m-1) \kappa d \cos (\theta),
$$

where, $\theta$ is the angle at which the source signal is impinging on the array. $d$ is the separation between two adjacent sensors. Now, the signal at each sensor is given by

$$
y_{m}(t)=\alpha e^{j(\omega t-\delta t)} .
$$

The vector form of the equation is given by

$$
\boldsymbol{y}(t)=\left[\begin{array}{c}
y_{1}(t) \\
y_{2}(t) \\
\vdots \\
y_{M}(t)
\end{array}\right]=\left[\begin{array}{c}
1 \\
e^{-j \kappa d \cos \theta} \\
\vdots \\
e^{-j(M-1) \kappa d \cos \theta}
\end{array}\right] x(t) .
$$

For $K$ sources, the equation is given by:

$$
\boldsymbol{y}(t)=\sum_{k=1}^{K} \boldsymbol{a}_{\theta_{k}} x_{k}(t) .
$$


Here,

$$
\boldsymbol{a}_{\theta_{k}}=\left[\begin{array}{llll}
1 & e^{-j \kappa d \cos \theta_{k}} & \cdots & e^{-j(M-1) \kappa d \cos \theta_{k}}
\end{array}\right]^{T} .
$$

$\boldsymbol{a}_{\theta_{k}}$ is the "steering vector" for direction $\theta_{k}$. All the steering vectors, when stacked inside a matrix, form and array manifold matrix, $\boldsymbol{A}_{\Theta}$. Where, $\Theta=\left[\begin{array}{llll}\theta_{1} & \theta_{2} & \cdots & \theta_{k}\end{array}\right]$. $\boldsymbol{A}_{\Theta}$ is given by:

$$
\boldsymbol{A}_{\Theta}=\left[\begin{array}{llll}
\boldsymbol{a}_{\theta_{1}} & \boldsymbol{a}_{\theta_{2}} & \cdots & \boldsymbol{a}_{\theta_{K}}
\end{array}\right]
$$

The received signal at time $t$, is given by

$$
\boldsymbol{y}(t)=\boldsymbol{A}_{\Theta} \boldsymbol{x}(t)+\boldsymbol{\eta}(t),
$$

where, $\boldsymbol{\eta}(t)=\left[\begin{array}{llll}\eta_{1} & \eta_{2} & \cdots & \eta_{M}\end{array}\right]$ is a vector representing additive noise at the output of each sensor. The noise is modeled as having a Gaussian distribution with mean $\boldsymbol{\mu}$ and covariance matrix $\boldsymbol{\Sigma}=\sigma^{2} \boldsymbol{I}$. And the source vector $\boldsymbol{x}(t)=$ $\left[\begin{array}{llll}x_{1}(t) & x_{2}(t) & \cdots & x_{K}(t)\end{array}\right]$.

For the discrete case, when the analog signal is sampled with a frequency $F_{s}=$ $\frac{1}{T_{s}}$, the receiver model is given by the following equation:

$$
\boldsymbol{y}(n)=\boldsymbol{A}_{\Theta} \boldsymbol{x}(n)+\boldsymbol{\eta}(n) .
$$

Here, $n=\frac{t}{T_{s}}$, where, $T_{s}$ is the sampling time period, corresponding to the sampling frequency, $F_{s}$.

Equation (2.18) is the model that is used throughout this thesis.

The distance between the signal source and the center of antenna array is assumed to be at least $10 \lambda$, so that the waves impinging on the antenna array can be considered as planar and in far field. 


\subsection{Array Signal Processing}

In this section, we discuss some of the existing algorithms for estimation of direction of arrival. Direction of arrival estimation is divided into two main categories based on the signal bandwidth. The first category is the the narrowband DOA estimation and the second is the broadband or wideband DOA estimation. The difference lies in the frequency spectrum of the signal. If the signal contains a very narrow band of frequencies and can be estimated as a single frequency, it is referred to as a narrowband signal. But if the signal contains a wide band of frequencies, usually more than 10 percent of the center frequency, is referred to as a broadband signal. The signals can be differentiated based on the fractional bandwidth, $\delta f$ given by the following equation:

$$
\delta f=\frac{f_{u}-f_{l}}{0.5\left(f_{u}+f_{l}\right)} .
$$

$f_{l}$ is the lowest frequency contained in the signal, while, $f_{u}$ represents the highest frequency contained in the signal. If $\delta f \ll 0.1$ the signal is called narrowband signal. If $\delta f>0.1$, the signal is called broadband signal.

\subsection{Narrowband DOA Estimation}

In this section, we discuss some of the well known DOA estimation techniques for narrowband signals. 


\subsubsection{Beamforming}

Beamforming is a technique used for summing up signal of interest while suppressing the noise. The output of a scanning beamformer is a spectrum displaying strength of the beamformer for each direction. A peak is observed at the direction at which the source is present. One of the beamformers, called delay-and-sum beamformer, has its direction finding function given by the following equation:

$$
\Gamma_{d n s}(\theta)=\boldsymbol{A}^{H} \boldsymbol{y}
$$

Here, the matrix $\boldsymbol{A}$ is an expanded version of the array manifold matrix in (2.16), as it contains steering vectors for all the directions of interest, instead of just containing the directions of the source signals. As the assumption is that we don't have the knowledge of the directions from which the source signals are arriving.

\subsubsection{The MVDR Algorithm}

Minimum Variance Distortion-less Response (MVDR) or Capon beamformer [8] is a popular algorithm used to estimate DOA. This algorithm does not require prior knowledge of the number of sources. Assume that we have $r$ linearly independent measurements, $\boldsymbol{Y} \in \mathbb{C}^{M \times r}$, such that $\boldsymbol{Y}=\boldsymbol{A} \boldsymbol{X}+\boldsymbol{H}$. Here, $r \geq K$. Then, we can compute the covariance matrix from the measurements in the following manner:

$$
\boldsymbol{R}=\frac{1}{r} \sum_{i=1}^{r} \boldsymbol{y}_{i} \boldsymbol{y}_{i}^{T}
$$

The MVDR algorithm forms a spectrum, in which source locations are indicated by peaks. The solution is as follows,

$$
\Gamma_{m v d r}(\theta)=\frac{\boldsymbol{a}_{\theta}^{H} \boldsymbol{a}_{\theta}}{\boldsymbol{a}_{\theta}^{H} \boldsymbol{R}^{-1} \boldsymbol{a}_{\theta}} .
$$


We can see the occurrence of $\boldsymbol{R}^{-1}$ in the solution. For rank-deficient covariance matrix, $\boldsymbol{R}$, the inverse does not exist. This algorithm cannot be used when we have a degenerate covariance matrix.

\subsubsection{The MUSIC Algorithm}

The MUSIC algorithm is used to estimate parameters such as DOA or signal spectrum. This algorithm was proposed by Schmidt [29] and is a benchmark for high resolution DOA estimation algorithms. The original form of the algorithm is formulated for continuous DOA parameter, $\theta$. However, Bresler [32] modified the criterion to identify support set of discrete DOA from a finite dictionary. This algorithm is based on eigen decomposition of the computed covariance matrix of the snapshots. Using singular value decomposition, the matrix $\boldsymbol{R}$, given by (2.21), can be decomposed into signal subspace, $\boldsymbol{U}$, and noise subspace, $\boldsymbol{Q}$ :

$$
\boldsymbol{R}=\left[\begin{array}{ll}
\boldsymbol{U} & \boldsymbol{Q}
\end{array}\right] \operatorname{diag}\left[\sigma_{1}, \ldots, \sigma_{K}, \sigma_{0}, \ldots, \sigma_{0}\right] \boldsymbol{V}^{H}
$$

Then, for any $j \in\{1, \ldots, N\}$, where $N$ represents the number of directions, $j \in$ $\operatorname{supp} \boldsymbol{X}$ if and only if [25]

$$
\boldsymbol{Q} \boldsymbol{a}_{j}=0
$$

or equivalently,

$$
\boldsymbol{a}_{j} P_{Q} \boldsymbol{a}_{j}=0
$$

Where, $\operatorname{supp} \boldsymbol{X}=\left\{1 \leq i \leq n: \boldsymbol{x}_{i} \neq 0\right\} . \boldsymbol{a}_{j}$ is the discrete equivalent of the steering vector $\boldsymbol{a}_{\theta}$, given by (2.15). $P_{(.)}$denotes orthogonal projection matrix onto the subspace defined by the columns of the matrix that is in the subscript.

The original MUSIC spectrum [29] is given by the following equation: 


$$
\Gamma_{m u s i c}(\theta)=10 \log _{10} \frac{1}{\left\|\boldsymbol{Q}^{H} \boldsymbol{a}_{\theta}\right\|_{2}^{2}} .
$$

When $r<K$ the MUSIC equation of (2.24) does not hold. This is a drawback of the MUSIC algorithm as compared to Compressed Sensing based MMV algorithms, that are discussed later in this chapter.

\subsubsection{The MUSIC-like Algorithm}

Ng et al. [33] showed that the MUSIC algorithm can be formulated as a beamformer:

$$
\begin{aligned}
\underset{\boldsymbol{w}_{\theta}^{M U S}}{\operatorname{minimize}} & \left\|\boldsymbol{w}_{\theta}^{M U S}-\boldsymbol{a}_{\theta}\right\|_{2}^{2}, \\
\text { s.t. } & \boldsymbol{Q}^{H} \boldsymbol{w}_{\theta}^{M U S}=0 .
\end{aligned}
$$

Here, $\boldsymbol{a}_{\theta}$ is the steering vector (2.15). $\boldsymbol{w}_{\theta}^{M U S}$, is the MUSIC algorithm counterpart of the beamforming weight vector. The solution to this optimization problem is given by:

$$
\Gamma_{m u s i c}(\theta)=10 \log _{10} \frac{1}{\left\|\boldsymbol{Q}^{H} \boldsymbol{a}_{\theta}\right\|_{2}^{2}} .
$$

Where, $\Gamma_{\text {music }}(\theta)$ represents MUSIC pseudo-spectrum for direction $\theta$ and $\boldsymbol{Q}$ is the noise subspace $(2.23)$.

Based on the above representation of the MUSIC algorithm as a beamformer, the MUSIC-like algorithm proposed in [28] is a solution to the following beamforming optimization problem:

$$
\begin{aligned}
\underset{\boldsymbol{w}_{\theta}}{\operatorname{minimize}} & \boldsymbol{w}_{\theta}^{H} \boldsymbol{R} \boldsymbol{w}_{\theta} \\
\text { s.t. } & \boldsymbol{w}_{\theta}^{H}\left\{\boldsymbol{a}_{\theta} \boldsymbol{a}_{\theta}^{H}+\beta \boldsymbol{I}\right\} \boldsymbol{w}_{\theta}=c
\end{aligned}
$$


where $c, \beta>0$ are constants. $\boldsymbol{R}$ is the covariance matrix. Its solution is found by solving the following generalized eigenvalue problem:

$$
\boldsymbol{R} \boldsymbol{w}_{\theta}=\lambda_{\min }\left\{\boldsymbol{a}_{\theta} \boldsymbol{a}_{\theta}^{H}+\beta \boldsymbol{I}\right\} \boldsymbol{w}_{\theta}, \theta \in(0, \pi]
$$

The solution depends on the value of parameter $\beta$. However, the parameter $c$ in (2.29) has no consequence on the final solution, as its value is implicitly regulated by the optimization problem. The bounds for $\beta$ have been derived in [28] and [31]. The bounds depend on the inverse of the covariance matrix, $\boldsymbol{R}$. We will see how we can derive bounds for $\beta$ that work for rank-deficient covariance matrix, the inverse of which does not exist.

The source directions are identified through peaks occurring in the pseudospectrum given by the following function:

$$
\Gamma_{\text {music-like }}(\theta)=10 \log _{10} \frac{1}{\left|\boldsymbol{w}_{\theta}^{H} \boldsymbol{a}_{\theta}\right|}
$$

This formulation of the pseudo-spectrum is henceforth referred to as formulation 1.

\subsection{Broadband DOA Estimation}

In case of broadband sources, the received signal $\boldsymbol{y}$ at the sensor array is dependent on frequency, $f$, and can be modeled by the following equation:

$$
\boldsymbol{y}(f)=\boldsymbol{A}(f, \Theta) \boldsymbol{x}+\boldsymbol{\eta}
$$

There are several high resolution techniques to estimate the DOA of broadband signals. Below, we discuss some of the popular techniques. 


\subsubsection{Incoherent Signal Subspace Method (ISSM)}

The technique [34] used for broadband DOA estimation based on MUSIC principle is to incoherently sum up the spectra obtained by repeated application of MUSIC algorithm to different frequency sub-bands of the steering vector. The word incoherent is used because there is no preprocessing involved in the summation process, rather, by using divide and rule strategy multiple narrow-band DOA estimation problems are solved and then the outputs are combined. The essence of this method is captured by the following equation:

$$
\Gamma_{I S S M}=\frac{1}{\frac{1}{B W \times(m-k)} \sum_{f_{i}=f_{\text {min }}}^{f_{\max }} \boldsymbol{a}^{H}\left(f_{i}, \theta\right) P_{\boldsymbol{Q}_{f_{i}}} \boldsymbol{a}\left(f_{i}, \theta\right)} .
$$

Here, $\boldsymbol{Q}_{f_{i}}$ is the noise subspace for $i^{t h}$ frequency band. BW denotes the number of frequency bins across the signal bandwidth $\left[f_{\min }, f_{\max }\right] \cdot \boldsymbol{a}\left(f_{i}, \theta\right)$ represents the frequency dependent steering vector for frequency $f_{i}$ and direction $\theta$.

If, instead of arithmetic mean, the integration is performed using the geometric mean, the spectrum is represented by the following equation:

$$
\Gamma_{I S S M-G}=\frac{1}{\frac{1}{m-k} \prod_{f_{i}=f_{\min }}^{f_{\max }}\left(\boldsymbol{a}^{H}\left(f_{i}, \theta\right) P_{\boldsymbol{Q}_{f_{i}}} \boldsymbol{a}\left(f_{i}, \theta\right)\right)^{\frac{1}{B W}} .}
$$

Both methods have some pros and cons. The arithmetic mean approach is less error prone but has poor resolution, while the geometric mean one is more accurate than the former approach but with higher resolution. The performance of ISSM degrades if the spectrum is not flat and the sources are not coherent.

\subsubsection{Coherent Signal Subspace Method (CSSM)}

Hung et al. [39] developed a technique to coherently integrate signal from multiple frequency bands and then apply DOA estimation algorithms on the integrated sig- 
nal. Using a reference frequency $f_{0}$, focusing matrices are formed for each frequency bin $f_{i}$, that transform the array output, to become

$$
\boldsymbol{z}(i)=\boldsymbol{T}\left(f_{i}, \hat{\Theta}\right) \boldsymbol{y}(i)
$$

$\hat{\Theta}$ is a vector of approximate directions in which the sources are present. These directions are approximated with narrowband beamforming or DOA estimation methods like MVDR or MUSIC.

$\boldsymbol{T}\left(f_{i}, \hat{\Theta}\right)$ is the transformation matrix obtained using the following focusing equation:

$$
\boldsymbol{T}\left(f_{i}, \hat{\Theta}\right) \boldsymbol{A}\left(f_{i}, \Theta\right)=\boldsymbol{A}\left(f_{0}, \Theta\right) .
$$

Substituting (2.32) into (2.35), we have

$$
\boldsymbol{z}(i)=\boldsymbol{T}\left(f_{i}, \hat{\Theta}\right) \boldsymbol{A}\left(f_{i}, \Theta\right) \boldsymbol{x}+\boldsymbol{T}\left(f_{i}, \hat{\Theta}\right) \boldsymbol{\eta}
$$

The covariance matrix of the transformed variable $\boldsymbol{z}(i)$ is given by

$$
\boldsymbol{R}_{\boldsymbol{z} \boldsymbol{z}}\left(\boldsymbol{f}_{\boldsymbol{i}}\right)=E\left\{\boldsymbol{z}(i) \boldsymbol{z}^{H}(i)\right\}
$$

The resultant covariance matrix $\boldsymbol{R}$ is obtained through summation of the transformed matrices $\boldsymbol{R}_{z z}(\boldsymbol{i})$,

$$
\boldsymbol{R}=\sum_{f_{i}=f_{\min }}^{f_{\max }} \boldsymbol{R}_{\boldsymbol{z z}}\left(f_{i}\right)
$$

The matrix $\boldsymbol{R}$, when expanded is given by

$$
\boldsymbol{R}=\boldsymbol{A}\left(f_{0}, \Theta\right)\left\{\sum_{f_{i}=f_{\min }}^{f_{\max }} \boldsymbol{R}_{x x}\left(\boldsymbol{f}_{i}\right)\right\} \boldsymbol{A}^{H}\left(f_{0}, \Theta\right)+\boldsymbol{R}_{\eta},
$$

where, $\boldsymbol{R}_{x x}\left(\boldsymbol{f}_{i}\right)$ is the source covariance matrix for frequency band $f_{i}$. For uncorrelated zero-mean white Gaussian noise with variance $\sigma^{2}$, the matrix $\boldsymbol{R}_{\eta}$ is given by

$$
\boldsymbol{R}_{\eta}=\sum_{f_{i}=f_{\min }}^{f_{\max }} \sigma^{2} \boldsymbol{T}\left(f_{i}, \hat{\Theta}\right) \boldsymbol{T}^{H}\left(f_{i}, \hat{\Theta}\right)
$$


Now, the noise subspace of the matrix pencil $\left(\boldsymbol{R}, \boldsymbol{R}_{\eta}\right)$ is orthogonal to $\boldsymbol{A}\left(f_{0}, \bar{\Theta}\right)$. $\boldsymbol{A}\left(f_{0}, \bar{\Theta}\right)$ contains the steering vectors from true source directions, $\bar{\Theta}$.

\subsection{Compressive Sensing (CS)}

The conventional approach in sampling of signals follows the Shannon theorem which states that the sampling rate/frequency (also called Nyquist rate) must be at least twice the highest frequency contained in the signal. This principle is obeyed in analog to digital converters (ADCs), digital cameras, radar receivers and many other applications.

The theory of compressive sensing [13], however, is built on another property of the signal: the sparsity. It asserts that one can recover signal from far fewer samples than what the conventional approach dictates. Another important aspect of the CS theory is incoherence which pertains to the sensing method used. Sparsity means that the information rate of a continuous time signal may be much smaller than that suggested by its bandwidth. In other words, when expressed in appropriate basis $\boldsymbol{\Psi}$, we would need far less information to represent the signal as compared to the Nyquist rate requirement. While, Incoherence, says that the sensing waveform has extremely dense representation in $\boldsymbol{\Psi}$, but are spread out in the domain of the signal of interest.

The information about a signal $x(t)$ is obtained by linear functionals and the values are given by

$$
\boldsymbol{y}(k)=\left\langle\boldsymbol{x}, \boldsymbol{\Phi}_{k}\right\rangle, \quad k=1, \ldots, M
$$

$\boldsymbol{\Phi}_{k}$, represents the sensing waveform. For time domain sampling of analog one dimensional signals, $\boldsymbol{\Phi}_{k}$ is Dirac delta function. CS deals with scenarios when the 
signal $y$ is under-sampled, such that the number of samples $M$ are significantly less than the dimension of the signal $N$. The reasons for under-sampling are the limitations in resources, e.g., channel capacity limitations, pricing limitations (in case of Magnetic Resonance Imaging), etc.

Let $\boldsymbol{A}$ denote sensing matrix of dimension $M \times N$, where $M \ll N$. Then the equation, $\boldsymbol{y}=\boldsymbol{A} \boldsymbol{x}$, is under-determined and can have infinitely many solutions. To find a unique solution to this problem, the signal $\boldsymbol{x}$, should be sparse in domain $\boldsymbol{\Psi}$ and additionally the sensing domain $\boldsymbol{\Phi}$ should be incoherent with the sparse domain.

The coherence between the representation system $\boldsymbol{\Psi}$ and the sensing system $\boldsymbol{\Phi}$ is given by $\mu$, where,

$$
\mu(\boldsymbol{\Phi}, \boldsymbol{\Psi})=\sqrt{N} \max _{1 \leq k, j \leq N}\left|\left\langle\boldsymbol{\Phi}_{k}, \boldsymbol{\Psi}_{j}\right\rangle\right|
$$

The coherence, $\mu$, is the measure of the largest correlation between any two elements of $\boldsymbol{\Phi}$ and $\boldsymbol{\Psi}$, while, $\mu(\boldsymbol{\Phi}, \boldsymbol{\Psi}) \in[1, \sqrt{N}]$. Since, $\left\langle\boldsymbol{\Phi}_{k}, \boldsymbol{\Psi}_{j}\right\rangle$ is an inner product and is less than or equal to one, the upper end point becomes $\sqrt{N}$. The lower endpoint is unity because the Parseval's theorem states that for each $j, \sum_{k=1}^{N}\left|\left\langle\boldsymbol{\Phi}_{k}, \boldsymbol{\Psi}_{j}\right\rangle\right|=$ $\left\|\Psi_{j}\right\|_{2}^{2}=1$. Compressive sensing works only for low coherence pairs of $\boldsymbol{\Phi}$ and $\boldsymbol{\Psi}$. One such pair, which we have used, is when $\boldsymbol{\Phi}=\boldsymbol{I}$ and $\boldsymbol{\Psi}=\boldsymbol{F}$, where $\boldsymbol{I}$ represents identity matrix and $\boldsymbol{F}$ represents the Fourier matrix. The incoherence for this pair is $\mu(\boldsymbol{\Phi}, \boldsymbol{\Psi})=1$. The reason for this choice will become apparent in the next chapter.

For exact recovery of the signal $\boldsymbol{x}, l_{1}$-minimization is used, as given by the following optimization problem: 


$$
\begin{array}{ll}
\underset{\hat{\boldsymbol{x}}}{\operatorname{minimize}} & \|\hat{\boldsymbol{x}}\|_{l_{1}}, \\
\text { subject to } & \boldsymbol{y}=\boldsymbol{\Psi} \boldsymbol{\Phi} \boldsymbol{x} .
\end{array}
$$

The following theorem gives the conditions for the exact recovery of the signal, $\boldsymbol{x}$, when we have the model: $\boldsymbol{y}=\boldsymbol{\Psi} \boldsymbol{\Phi} \boldsymbol{x}$.

Theorem 1:[20] Fix $\boldsymbol{f} \in \mathcal{R}^{N}$ and suppose that the coefficient sequence $\boldsymbol{x}$ of $\boldsymbol{f}$ in the basis $\boldsymbol{\Phi}$ is S-sparse. Select $M$ measurements in the $\boldsymbol{\Psi}$ domain uniformly at random, then if

$$
M \geq C \mu^{2}(\boldsymbol{\Phi}, \boldsymbol{\Psi}) \operatorname{Slog}(N)
$$

for some positive constant $C$, the solution to (2.44) is exact with overwhelming probability.

This theorem takes care of the noiseless case. For a more robust case where there is additive noise in the system the model is given by

$$
\boldsymbol{y}=\boldsymbol{A x}+\boldsymbol{\eta},
$$

where, $\boldsymbol{\eta}$, represents the additive noise, and $\boldsymbol{A}=\boldsymbol{\Phi} \boldsymbol{\Psi}$. For each integer $s=1,2, \ldots$, define the isometry constant $\delta_{s}$ of a matrix $\boldsymbol{A}$ as the smallest number such that

$$
\left(1-\delta_{s}\right)\|\boldsymbol{x}\|_{2}^{2} \leq\|\boldsymbol{A} \boldsymbol{x}\|_{2}^{2} \leq\left(1+\delta_{s}\right)\|\boldsymbol{x}\|_{2}^{2},
$$

holds for all sparse vectors $x$. Matrix $\boldsymbol{A}$ obeys Restricted Isometry Property (RIP) of order $s$ if $\delta_{s}$ is not too close to 1 . The RIP condition ensures that s-sparse signals would not reside in the null space of $\boldsymbol{A}$, otherwise, it would not be recoverable.

For $(2.46)$, the following $l_{1}$-minimization guarantees exact recovery of the signal 
under the noisy conditions:

$$
\begin{aligned}
& \underset{\hat{\boldsymbol{x}}}{\operatorname{minimize}} \quad\|\hat{\boldsymbol{x}}\|_{l_{1}} \\
& \text { subject to }\|\boldsymbol{y}-\boldsymbol{A} \hat{\boldsymbol{x}}\|_{l_{2}} \leq \epsilon \text {. }
\end{aligned}
$$

Theorem 2: [12] Assume that $\delta_{2 S}<\sqrt{2}-1$. Then the solution $\boldsymbol{x}^{*}$ to $(2.48)$ obeys

$$
\left\|\boldsymbol{x}^{*}-\boldsymbol{x}\right\|_{l_{2}} \leq C_{0}\left\|\boldsymbol{x}^{*}-\boldsymbol{x}_{S}\right\|_{l_{1}} / \sqrt{S}+C_{1} \epsilon
$$

For a vector $\boldsymbol{x}$ in $\mathcal{R}^{N}, \boldsymbol{x}_{\boldsymbol{S}}$ denotes its best S-sparse approximation; that is, $\boldsymbol{x}_{\boldsymbol{S}}$ is the approximation obtained by keeping the $\mathrm{S}$ largest entries of $\boldsymbol{x}$ and setting the the rest to zero.

The reconstruction error $\left\|\boldsymbol{x}^{*}-\boldsymbol{x}\right\|_{l_{2}}$ is bounded by summation of two terms. The first term is the error if there is only noiseless data, the second term is the noise contribution. The constants $C_{0}$ and $C_{1}$ are typically small. This result establishes that CS can work in noisy conditions as well and is robust.

In the following section we present greedy CS algorithms that are relevant to this thesis.

\subsubsection{Simultaneous Orthogonal Matching Pursuit (SOMP)}

Simultaneous Orthogonal Matching Pursuit (SOMP) [16] is a greedy CS algorithm that is applied to multiple measurement vectors. Similar to Basis Pursuit algorithm, this algorithm can recover the signal, from severely undersampled signal. The S-OMP [16] algorithm is given below:

i Inputs: $\boldsymbol{Y}$. 
ii At first Iteration set residual matrix $\boldsymbol{B}_{0}=\boldsymbol{Y}$ and index set $\boldsymbol{S}_{0}=\emptyset$.

iii Find an index $s_{j}$ that solves the optimization problem $\left\|\boldsymbol{a}_{s_{J+1}}^{*} \boldsymbol{B}_{J}\right\|_{2}=\max _{1 \leq s \leq N}\left\|\boldsymbol{a}_{s}^{*} \boldsymbol{B}_{J}\right\|_{2}$.

iv Calculate $\boldsymbol{B}_{J}=\left(\boldsymbol{I}-P_{\boldsymbol{S}_{J}}\right) \boldsymbol{Y}$, here $P_{\boldsymbol{S}_{J}}$ is the orthogonal projection onto span of the vectors $\left\{\boldsymbol{a}_{s_{j}}\right\}_{j=1}^{J}$.

Where $s \in\{1, \cdots, N\}$. N represents the number of directions in which the DOA space is discretized.

\subsubsection{MUSIC with SOMP Support}

Kim et. al. [25] proved that if we have $r$ snapshots and $K$ sources and $r \leq K$, and $K-r$ index support is available using one of the many Multiple Measurement Vector (MMV) algorithms, then the remaining $r$ sources could be estimated deterministically using a variation of MUSIC algorithm given by the "generalized MUSIC criterion (GMC)":

$$
\boldsymbol{a}_{j}^{*}\left[P_{Q}-P_{Q Q^{*}} \boldsymbol{A}_{\boldsymbol{I}_{K-r}}\right] \boldsymbol{a}_{j}=0
$$

Here, $\boldsymbol{Q}$ represents the noise subspace. $P_{(.)}$represents orthogonal projection matrix on to the subspace defined by columns of a matrix. $\boldsymbol{A}_{\boldsymbol{I}_{K-r}}$ is a matrix containing direction vectors from $K-r$ source directions that have indices $\boldsymbol{I}_{K-r} . \boldsymbol{I}_{K-r}$ is a vector containing $K-r$ estimated indices. These $K-r$ sources are estimated using MMV algorithm like S-OMP.

In contrast, the MUSIC criterion is just

$$
\boldsymbol{a}_{j}^{*}\left[P_{Q}\right] \boldsymbol{a}_{j}=0
$$

but it is only applicable when $r \geq M$. 


\section{Chapter 3}

\section{Recursive MUSIC-Like Algorithm}

\section{for DOA Estimation of}

\section{Narrowband Sources}

\subsection{Introduction}

The approach used to estimate the direction of arrival (DOA) of source signals is to use an array of antenna sensors. In the case of far-field and narrow band sources, the wave-fronts emanating from a source can be approximated as planes of amplitude. By appropriate weighting mechanism (gain/phase) for each look direction, the array outputs can be summed together to generate a peak at the directions of the origin of the sources. This mechanism, however, is low-resolution. It cannot discriminate between two (or more) sources that are located in close proximity of each other (here the close proximity is based on direction, as measured from the perspective 
of the array of sensors).

To enhance resolution of closely located sources, super resolution techniques such as multiple signal classification (MUSIC) [29] and estimation of signal parameters via rotational invariance technique (ESPRIT) [30] are very popular. These techniques require subspace augmentation into signal and noise subspaces. The dimension of the signal subspace can be estimated through different techniques. But an error in subspace dimension, usually produce unwanted results. Y. Zhang and B. P. Ng proposed a MUSIC-like algorithm [28] which estimates the direction of arrival (DOA) of multiple sources without the knowledge of the number of sources. Similar to the MUSIC algorithm, this algorithm assumes that the covariance matrix is full-rank. If the covariance matrix is rank-deficient, this algorithm fails to estimate even a single source. When the covariance matrix is full rank, the maximum number of sources these algorithms can estimate is one less than the number of sensors. This bound is higher than what the MMV CS algorithms can achieve.

Simultaneous Orthogonal Matching Pursuit (S-OMP) is a greedy algorithm, and is the MMV counterpart of the Orthogonal Matching Pursuit (OMP) algorithm. It has been discussed briefly in Section 2.6.1. This algorithm combines the multiple measurement vectors to estimate the sources present. It is an iterative algorithm, and operates without the knowledge of the number of sources.

In [25], Kim et al. proposed an algorithm which is a hybrid of SOMP and MUSIC algorithm. This algorithm has been previously discussed in Section 2.6.2. Something similar was proposed by K. Lee and Y. Bresler [27], they called it Subspace Augmented MUSIC (SA-MUSIC). When the number of snapshots are less than the number of sources present, the covariance matrix becomes rank deficient. Using traditional MUSIC algorithm, we are unable to estimate the directions of source signals impinging on the antenna array. What Kim et al. have suggested is 
to use SOMP or 2-thresholding algorithm to estimate some source directions and the remaining source directions are estimated using a special kind of MUSIC algorithm called generalized MUSIC criterion. One drawback of this approach is that it presupposes that we have exact knowledge of the number of sources.

In this Chapter, we study the problem of estimating direction of arrival of signals from multiple measurement vectors, without the knowledge of the number of sources and with rank deficient covariance matrix. We also derive the bounds of a parameter, that is significant to the working of MUSIC-like algorithm. The parameter, in its current form, cannot be used when the covariance matrix is rank deficient. We also suggest a formulation of the MUSIC-like algorithm in a way similar to MUSIC algorithm, which offer certain advantages that the original derivation lacks.

\subsection{Multiple Measurement Vectors}

Suppose there are a number of narrowband signals originating from $K$ directions and we have $M$ sensors that form an array. The elements of the array are placed in a straight line. $\lambda$ is the wavelength corresponding to the center frequency, $f_{c}$, of the receiver. These sensors span a line (one dimension) and are thus capable of resolving direction of arrival of sources in one dimension (usually azimuth direction, represented by $\theta$ ). We assume that the signals are originating from any of the $N$ directions, in which we divide the DOA space. Then our received signal, $\boldsymbol{y}$ is given by

$$
\boldsymbol{y}=\boldsymbol{A x} .
$$


$\boldsymbol{y} \in \mathbb{C}^{M \times 1}$ is the measurement vector. $\boldsymbol{x} \in \mathbb{C}^{N \times 1}$ contains source signals at the time instant $t$. This vector is assumed to be sparse having just a few non-zero elements. That is,

$$
\|\boldsymbol{x}\|_{0}=k
$$

where $\|\cdot\|_{0}$ denotes the number of non-zero elements, or in other words, the sparsity level of the vector.

$\boldsymbol{A}$ contains vectors from $N$ directions as its columns. That is,

$$
\boldsymbol{A}=\left[\begin{array}{llllll}
\boldsymbol{a}_{1} & \cdot & \boldsymbol{a}_{i} & . & \boldsymbol{a}_{N}
\end{array}\right], \quad i \in\{1, \ldots, N\},
$$

and the steering vector is given by

$$
\boldsymbol{a}_{i}=e^{j \boldsymbol{\kappa}_{i}^{T} \boldsymbol{L}}=\frac{1}{\sqrt{m}}\left[e^{j \boldsymbol{\kappa}_{i}^{T} \boldsymbol{l}_{1}}, \ldots, e^{j \boldsymbol{\kappa}_{i}^{T} \boldsymbol{l}_{M}}\right]^{T},
$$

where $\boldsymbol{l}_{j}$ is the three dimensional position vector for $j^{t h}$ sensor and $\boldsymbol{\kappa}_{i}$ is the wavenumber vector for source $i$. It is given by $\boldsymbol{\kappa}_{i}=\frac{2 \pi}{\lambda} v_{\boldsymbol{\theta}_{i}} . v_{i}$ is a unit vector in the direction of $i^{\text {th }}$ source. This is the model when we have a single measurement vector (SMV). In Section 2.2, we have gone through similar derivation for the case when the DOA space is continuous. Most of the derivation is quite similar, but there is one marked difference. The difference is the dimension of the matrix $\boldsymbol{A}$. In this chapter, the matrix $\boldsymbol{A}$, contains steering vectors for all the possible directions, $N$, and is thus a fat matrix. While in Section 2.2 the array manifold matrix is a tall matrix and its number of columns is less than the number of rows.

For SMV case, Candes and Romberg [9] have proposed to use $l_{1}-n o r m$ instead of using $l_{0}-$ norm (which is a combinatorial problem and is NP hard). The theorem presented in [20] states that instead of solving the problem,

$$
\begin{array}{r}
\underset{\boldsymbol{x}}{\operatorname{minimize}} \quad\|\boldsymbol{x}\|_{0}, \\
\text { subject to } \quad \boldsymbol{y}=\boldsymbol{A x},
\end{array}
$$


if we solve the problem (this problem is called Basis Pursuit):

$$
\begin{aligned}
\underset{\boldsymbol{x}}{\operatorname{minimize}} & \|\boldsymbol{x}\|_{1}, \\
\text { subject to } & \boldsymbol{y}=\boldsymbol{A x},
\end{aligned}
$$

we are guaranteed to recover $\boldsymbol{x}$ with a very high probability. This problem now becomes a linear programming optimization problem which is relatively easy to solve. There are other methods in the literature to solve the problem of (3.5). There are a number of greedy algorithms that attempt to solve this problem. One of such algorithms is orthogonal matching pursuit (OMP). It has been proven by Donoho et. al. [14] and Tropp [15] independently that OMP can solve (3.5) under certain conditions.

For noisy systems the model is $\boldsymbol{y}=\boldsymbol{A} \boldsymbol{x}+\boldsymbol{\eta}, \boldsymbol{\eta} \in^{M \times 1}$ is a vector realization of random Gaussian process. Candes et al. [20] suggested a solution to this problem called Basis Pursuit De-Noising (BPDN), discussed previously in Section 2.6.

If there are more than one measurement vector, then we can reformulate the SMV problem to MMV problem, given by

$$
\begin{array}{r}
\underset{\boldsymbol{X}}{\operatorname{minimize}} \quad\|\boldsymbol{X}\|_{0}, \\
\text { subject to } \boldsymbol{Y}=\boldsymbol{A} \boldsymbol{X},
\end{array}
$$

where $\boldsymbol{X}=\left[\boldsymbol{x}_{1}, \ldots, \boldsymbol{x}_{r}\right] \in \mathbb{R}^{N \times r}, \boldsymbol{Y}=\left[\boldsymbol{y}_{1}, \ldots, \boldsymbol{y}_{r}\right] \in \mathbb{R}^{M \times r}$ and $\|\boldsymbol{X}\|_{0}=|\operatorname{supp} \boldsymbol{X}|$, where $\operatorname{supp} \boldsymbol{X}=\left\{1 \leq i \leq N: \boldsymbol{x}^{i} \neq 0\right\}$ and $\boldsymbol{x}^{i}$ is $i^{\text {th }}$ row of $\boldsymbol{X}$. Here, we have $r$ snapshots instead of one, as is the case in SMV model of Section 2.6. We can see this as multiple SMV problems, but we won't garner any advantage if we deal with these $r$ problems separately. It is indeed advantageous to solve MMV problem in a different manner. Tropp et al. have proposed a greedy algorithm called Simultaneous Orthogonal Matching Pursuit (S-OMP) [16] in which they solve 
the MMV problem as a single whole. There are other algorithms including mixed norm approach, REduce MMV and BOost(ReMBO), etc. which have addressed this problem. For detailed discussion of these algorithms and their merits and demerits please refer to $[26,25]$.

But when dealing with the DOA estimation problem, one invariably comes across noise modeled as a Gaussian process. When there is additive noise in the system we modify our model to the following

$$
\boldsymbol{Y}=\boldsymbol{A} \boldsymbol{X}+\boldsymbol{H}
$$

$\boldsymbol{H} \in \mathbb{C}^{M \times r}$ is a matrix containing complex noise vectors. The elements of $\boldsymbol{H}$ are assumed to be independent and identically distributed (i.i.d) random variables following the complex Gaussian distribution $\mathcal{C N}\left(0, \sigma^{2} \boldsymbol{I}\right)$.

We have discussed the classical DOA estimation algorithms like the MVDR algorithm and the MUSIC algorithm in Sections 2.4.2 and Section 2.4.3, respectively. The MVDR algorithm cannot be used for the case when $r<K$ as the covariance matrix becomes degenerate and the MVDR spectrum of (2.22) is dependent on inverse of the covariance matrix. The MUSIC algorithm also cannot be used, because of the degenerate covariance matrix as discussed previously. The SOMP algorithm (Section 2.6.1) and CS-MUSIC algorithm (Section 2.6.2) can be used with degenerate covariance matrix but they don't have the MMV integration capability like the MUSIC algorithm and the sparsity signal assumption for music is less strict as compared to these two algorithms. The MUSIC algorithm unlike SOMP relies on second order statistics of the probability distribution by first computing covariance matrix and then partitioning it into noise and signal subspaces that are orthogonal to each other. This process is lacking in SOMP algorithm which relies on finding local maximum of the cost function and then projecting the rest of the vectors onto 
the orthogonal space of the vector that maximizes the cost function. The sparsity level of MUSIC algorithm, for full-rank covariance matrix is $K<M-1$, while for SOMP it is significantly less than that. This makes the MUSIC algorithm superior to the CS algorithms discussed in Chapter 2. But in case of degenerate covariance matrix the MUSIC algorithm would fail.

Next we discuss the MUSIC-like algorithm in the context of the degenerate covariance matrix and Compressive Sensing.

\subsection{Recursive MUSIC-like (RML) Algorithm}

The MUSIC-like algorithm was originally designed, similar to the MUSIC algorithm, for estimation of continuous parameters such as direction of arrival. This algorithm can be modified somewhat to make it compatible with the MMV problem. The first step is to discretize the direction space, $\theta$, where $\theta \in(0, \pi]$. We have $N$ direction vectors contained in the matrix $\boldsymbol{A}$, given by (3.3). The MUSIC-like weight vectors can be computed in the following manner:

$$
\boldsymbol{R} \boldsymbol{w}_{i}=\lambda_{\min }\left\{\boldsymbol{a}_{i} \boldsymbol{a}_{i}{ }^{H}+\beta \boldsymbol{I}\right\} \boldsymbol{w}_{i}, i \in\{1, \ldots, N\}
$$

Here, $\boldsymbol{W}=\left[\boldsymbol{w}_{1}, \ldots, \boldsymbol{w}_{N}\right]$, where $\boldsymbol{w}_{i}^{\prime} s$ are found using (3.9). $\boldsymbol{a}_{i}$ is the steering vector for direction represented by index $i$, where $i \in\{1, \ldots, N\}$. $\boldsymbol{W}$ is MUSIClike counterpart of $\boldsymbol{Q}$, i.e. the noise subspace (2.23). The original formulation of MUSIC-like pseudo-spectrum given by (2.31) is somewhat different from that of MUSIC algorithm, given by:

$$
\Gamma_{\text {music }}(\theta)=10 \log _{10} \frac{1}{\left\|\boldsymbol{Q}^{H} \boldsymbol{a}_{\theta}\right\|_{2}^{2}}
$$


We can see that in MUSIC-like formulation 1 of pseudo-spectrum, there is a dot product of two vectors in the denominator. The two vectors being $\boldsymbol{a}_{\theta}$ and $\boldsymbol{w}_{\theta}$. But in case of MUSIC algorithm the steering vector, $\boldsymbol{a}_{\theta}$, is projected onto noise-subspace and then scalarized through pre-multiplying with $\boldsymbol{a}_{\theta}$. Similar pseudo-spectrum formulation can be devised for MUSIC-like algorithm as well.

Similar to MUSIC conditions $(2.24,2.25)$, we have the following conditions in case of MUSIC-like algorithm:

$$
\boldsymbol{W}^{H} \boldsymbol{a}_{j}=0
$$

or equivalently

$$
\boldsymbol{a}_{j}^{H} P_{\boldsymbol{W}} \boldsymbol{a}_{j}=0
$$

The pseudo-spectrum can be now computed using the following equation (henceforth, referred to as formulation 2)

$$
\Gamma_{\text {music-like }}(\theta)=10 \log _{10} \frac{1}{\left\|\boldsymbol{W}^{H} \boldsymbol{a}_{\theta}\right\|_{2}^{2}} .
$$

Note that this formulation of the pseudo spectrum is different from the formulation in [28]. In [28], only single vector $\boldsymbol{w}$ is used for the pseudo spectrum calculation. The main reason is that the problem was posed as a beamforming optimization problem similar to MVDR rather than the subspace augmentation problem used for derivation of the MUSIC algorithm. Equation (3.13), however, is similar to MUSIC pseudo spectrum, as we are using a vector subspace instead of a single vector, $\boldsymbol{w}$. The dimension of $\boldsymbol{W}$ is $M \times N$. N is much larger than the real noise subspace dimension, which equals $M-K$. But the dimension of matrix $\boldsymbol{W}$ is not important, as long as the space it spans is the same as the noise 


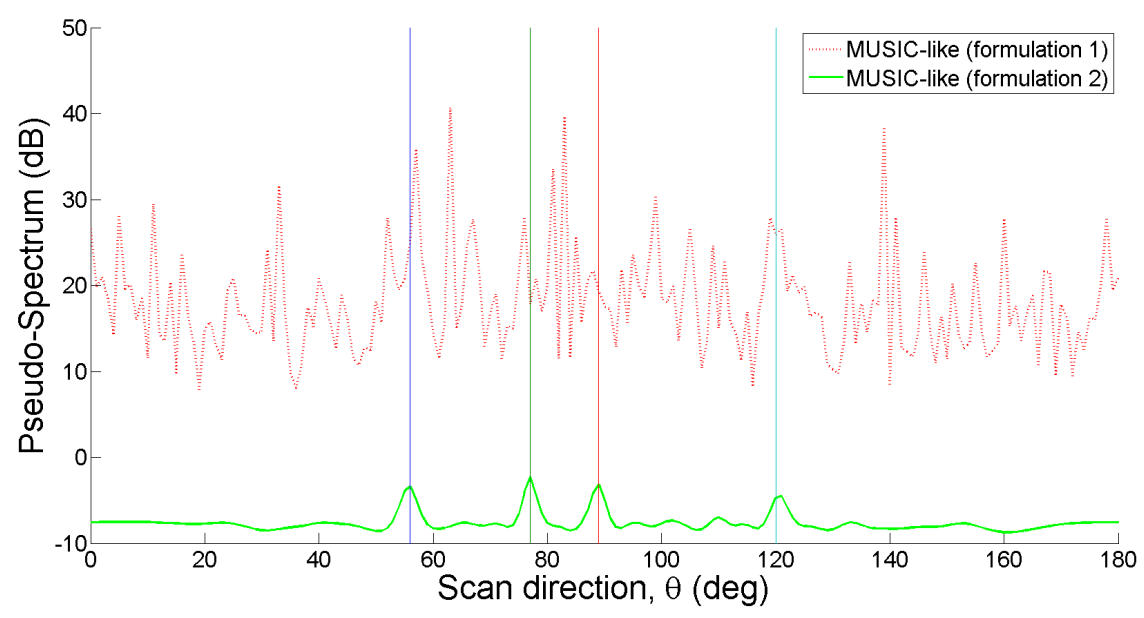

Figure 3.1: Comparison of DOA estimation capabilities of the two formulations of MUSIC-like algorithm for $\mathrm{r}=2, \mathrm{M}=31, \mathrm{~K}=4$ and $\mathrm{SNR}=40 \mathrm{~dB}$. The four vertical lines denote the locations of the actual sources located at 55, 78, 90 and 120 degrees.

subspace. In [28], the authors have already shown that the weights $\boldsymbol{w}$ reside in the noise subspace.

In Figure 3.1, we see the output of these two formulations when the covariance matrix is rank deficient. Note that we have set a very high SNR, but the formulation 1 failed to identify any peaks. In contrast, formulation 2 produced peaks at correct source locations.

For the MUSIC algorithm, we must know the exact dimension of the noise subspace, which is dependent on the knowledge of the number of sources. As we have seen above, the MUSIC-like algorithm does not presuppose the knowledge of the number of sources. When we have $r$ snapshots and also the condition that $r<K$, the covariance matrix, $\boldsymbol{R}$ is degenerate. The noise space, $\boldsymbol{W}$, is polluted 


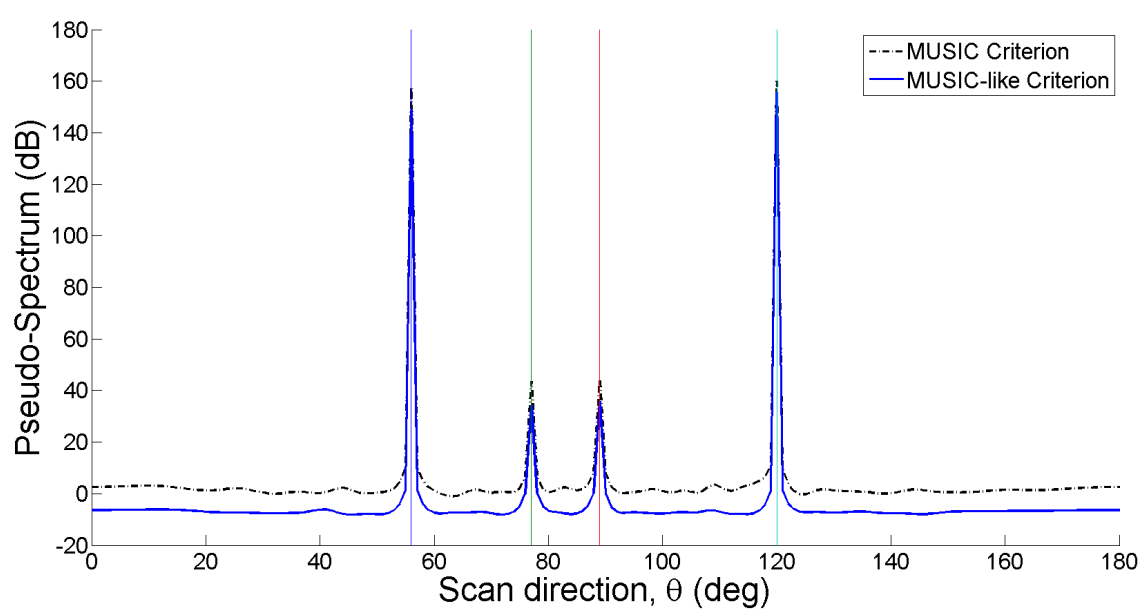

Figure 3.2: Pseudo-spectrum for generalized MUSIC criterion (2.50) and generalized MUSIC-like criterion (3.14) when $\mathrm{M}=31, \mathrm{~K}=4, \mathrm{r}=2$ and $\mathrm{SNR}=20 \mathrm{~dB}$.

with signal vectors as well. If we know the direction of $K-r$ sources, then the noise subspace can be obtained using a projection of $\boldsymbol{W}$ onto the subspace that is perpendicular to $\boldsymbol{A}_{\boldsymbol{I}_{K-r}}$. Here, $\boldsymbol{A}_{\boldsymbol{I}_{K-r}}$, is the direction manifold matrix containing only steering vectors from the known directions. The indices of the directions are contained in the matrix $\boldsymbol{I}_{K-r}$. We define the generalized MUSIC-like criterion $(G M L C)$ as following:

$$
\boldsymbol{a}_{j}^{H}\left[P_{\boldsymbol{W}}-P_{P_{\boldsymbol{W}} \boldsymbol{A}_{\boldsymbol{I}_{K-r}}}\right] \boldsymbol{a}_{j}=0 .
$$

According to this criterion, if we have $r<K$, we can still use MUSIC-like algorithm to estimate the rest of sources, if necessary support of $K-r$ sources are provided. The indices of the sources are contained in vector $\boldsymbol{I}_{K-r}$. While, $\boldsymbol{A}_{\boldsymbol{I}_{K-r}}$ matrix contains the vectors $\boldsymbol{a}_{i}$ where $i \in \boldsymbol{I}_{K-r}$.

Figure 3.2 compares the pseudo-spectrum generated by GMC [25] and GMLC 


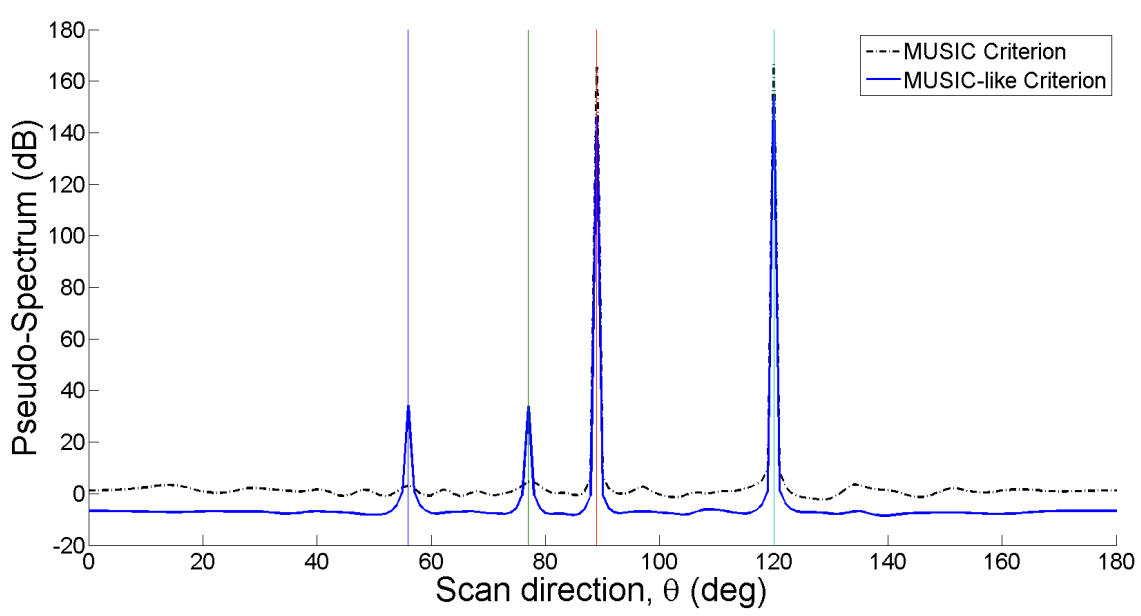

Figure 3.3: Comparison of generalized MUSIC criterion with generalized MUSIClike criterion when the estimate of dimension of $\boldsymbol{Q}$ is wrong.

(3.14). There are two very tall peaks for both criterion. These peaks occur at the directions $\theta_{i}$ where $i \in \boldsymbol{I}_{K-r}$. The other two directions were estimated. We can see from the figure that the performance of the two criteria are very similar, even when we have no explicit knowledge of the number of sources in the case of GMLC, while we had complete knowledge of the number of sources for GMC.

Figure 3.3 shows GMC and GMLC behavior when the estimate for the number of sources is incorrect. We can see that GMC has failed to detect any peaks other than the ones provided as support, while GMLC detected all the peaks successfully.

Although the GMLC works when we have not provided $K$ explicitly, but by providing $\boldsymbol{A}_{\boldsymbol{I}_{K-r}}$ as a support in GMLC, we are still providing implicit information about the number of sources.

This problem can be eliminated if we have a stand-alone algorithm based on GMLC that can provide initial index as well as the final source indices. 


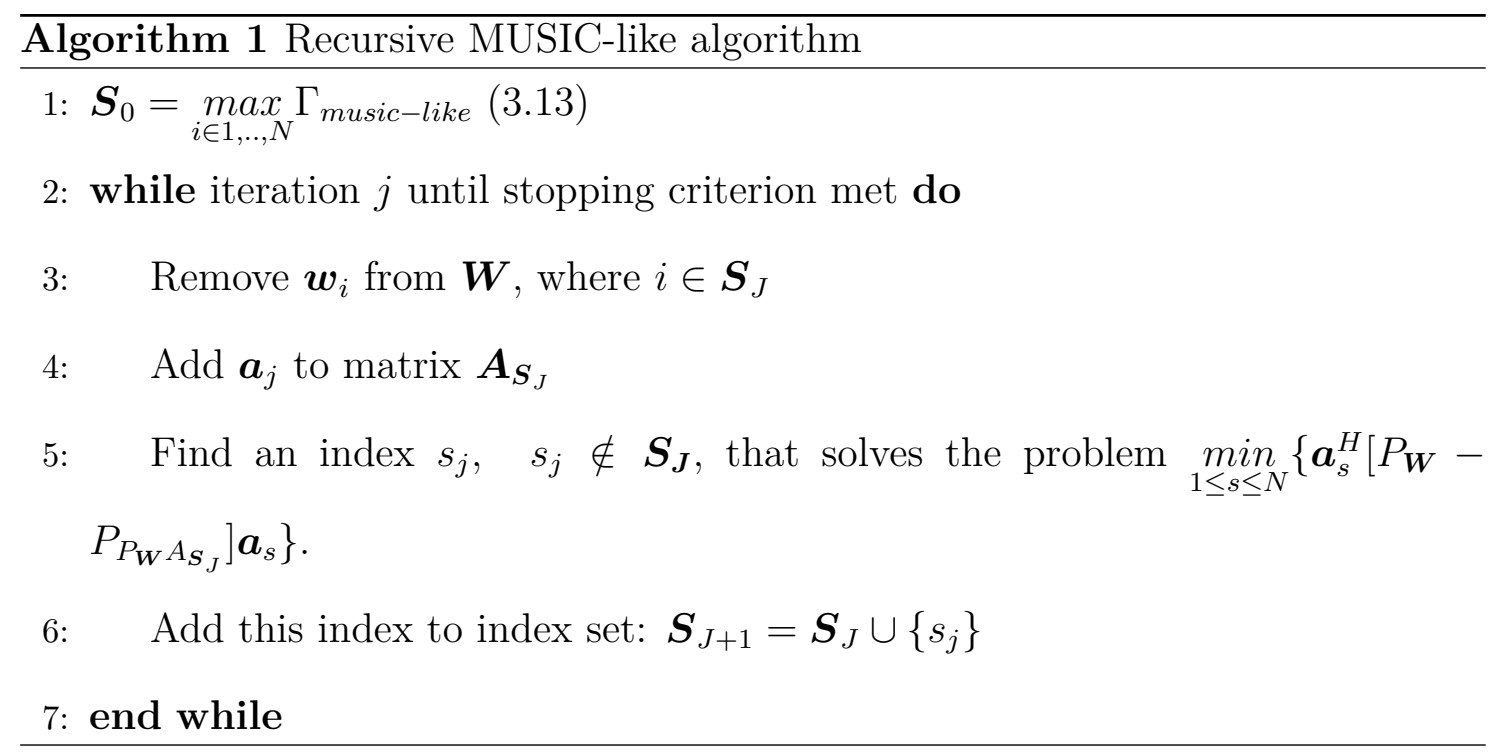

We propose an algorithm described as Algorithm 1. Initially using formulation 2 of MUSIC-like algorithm, we detect the highest peak in the pseudo-spectrum or the index that minimizes $\boldsymbol{a}_{j}^{H} P_{\boldsymbol{W}} \boldsymbol{a}_{j}$. This index is then added to index vector $\boldsymbol{S}_{0} . \boldsymbol{a}_{i}$ is then added to matrix $\boldsymbol{A}_{\boldsymbol{I}}$. The minimum value of GMLC is found for each iteration. The iterations are stopped when stoppage criterion is met. $\boldsymbol{S}$ now contains all the estimated indices of the sources.

This algorithm can now be used in a similar manner as MMV algorithms such as SOMP [16], SA-MUSIC [27] or MUSIC + SOMP [25].

\subsubsection{Stoppage Criterion for RML Algorithm}

Since it is an iterative algorithm we must supply a stoppage criterion. Here are the following possibilities:

i We iterate till the maximum iterations are reached. The value of maximum iterations is set to $\max$. 
ii At every iteration we find the index set $S_{J}$. If $\eta[J]<\mu$, we stop the iterations. Where

$$
\eta[J]=\frac{\left\|\left(\boldsymbol{I}-P_{\boldsymbol{A}_{\boldsymbol{S}_{J}}}\right) \boldsymbol{R}\right\|_{2}}{\left\|\boldsymbol{I}-P_{\boldsymbol{A}_{\boldsymbol{S}_{J}}}\right\|_{2}}
$$

$\mu$ is a measure of noise variance, which would be calculated next.

$\boldsymbol{I}-P_{\boldsymbol{A}_{\boldsymbol{S}_{J}}}$ is a matrix that projects any vector or matrix into the space perpendicular to that spanned by the columns of $\boldsymbol{A}_{\boldsymbol{S}_{J}}$. If we have correct $\boldsymbol{A}_{\boldsymbol{S}_{J}}$, then this projection would remove all source vectors and only noise would be left as a result.

The true covariance matrix of the measurements, $\boldsymbol{y}$ is given by

$$
\boldsymbol{\Sigma}_{y y}=\boldsymbol{A}_{S} \boldsymbol{\Sigma}_{s s} \boldsymbol{A}_{S}^{H}+\sigma_{v}^{2} \boldsymbol{I}_{M}
$$

In case the matrix $\boldsymbol{S}$ contains all source indices, we have $\boldsymbol{A}_{S}=\boldsymbol{A}_{\boldsymbol{S}_{J}}$. Then, $\left\|\left(\boldsymbol{I}-P_{\boldsymbol{A}_{\boldsymbol{S}_{J}}}\right) \boldsymbol{R}\right\|_{2}$, under approximation $\boldsymbol{R} \approx \boldsymbol{\Sigma}_{y y}$, becomes

$$
\left\|\left(\boldsymbol{I}-P_{\boldsymbol{A}_{S}}\right)\left(\boldsymbol{A}_{S} \boldsymbol{\Sigma}_{s s} \boldsymbol{A}_{S}^{H}+\sigma_{v}^{2} \boldsymbol{I}_{M}\right)\right\|_{2}
$$

Putting $P_{\boldsymbol{A}_{\boldsymbol{S}}}=\boldsymbol{A}_{\boldsymbol{S}}\left(\boldsymbol{A}_{\boldsymbol{S}}^{H} \boldsymbol{A}_{\boldsymbol{S}}\right)^{-1} \boldsymbol{A}_{\boldsymbol{S}}^{H}$ in (3.17), we get

$$
\left\|\left(\boldsymbol{I}-\boldsymbol{A}_{\boldsymbol{S}}\left(\boldsymbol{A}_{S}^{H} \boldsymbol{A}_{\boldsymbol{S}}\right)^{-1} \boldsymbol{A}_{\boldsymbol{S}}^{H}\right)\left(\boldsymbol{A}_{S} \boldsymbol{\Sigma}_{s s} \boldsymbol{A}_{S}^{H}+\sigma_{v}^{2} \boldsymbol{I}_{M}\right)\right\|_{2}
$$

After simplifying, (3.18) becomes

$$
\sigma_{v}^{2}\left\|\boldsymbol{I}-P_{\boldsymbol{A}_{S}}\right\|_{2}
$$

$\sigma_{v}^{2}$ is an unknown parameter. We can approximate it with $\lambda_{\boldsymbol{R}, \min }$, which we will see in the next section. Putting (3.19) in (3.15), we can get the inequality for parameter $\mu$ :

$$
\lambda_{\boldsymbol{R}, \min } \frac{\left\|\boldsymbol{I}-P_{\boldsymbol{A}_{\boldsymbol{S}_{J}}}\right\|_{2}}{\left\|\boldsymbol{I}-P_{\boldsymbol{A}_{\boldsymbol{S}_{J}}}\right\|_{2}}<\mu
$$


or,

$$
\lambda_{\boldsymbol{R}, \min }<\mu \text {. }
$$

Since the parameter $\mu$ is a thresholding parameter, we should set its value a bit higher than $\lambda_{\boldsymbol{R}, \min }$.

\section{When $R$ is Rank Deficient}

In case, matrix $\boldsymbol{R}$ is rank deficient and the assumption, $\boldsymbol{R} \approx \boldsymbol{\Sigma}_{y y}$, is not valid, some of the equations in the previous section needs to be reevaluated. Although, the rank deficient matrix $\boldsymbol{R}$ can no longer be decomposed into signal and noise subspaces, the results for the parameter $\mu$ would still hold, as we will see next.

Instead of (3.16), we have:

$$
\boldsymbol{R}=\boldsymbol{A}_{S} \hat{\boldsymbol{\Sigma}}_{s s} \boldsymbol{A}_{S}^{H}+\hat{\sigma}_{v}^{2} \boldsymbol{I}_{M}
$$

when the matrix $\boldsymbol{R}$ is rank-deficient.

Note that the true source covariance matrix, $\Sigma_{s s}$, has been replaced by the approximation, $\hat{\boldsymbol{\Sigma}}_{s s}$ and $\sigma_{v}^{2}$ has been replaced by $\hat{\sigma}_{v}^{2}$, due to the fact the the number of snapshots are not enough for the measurement covariance matrix, $R$, to be approximated as the true covariance matrix, $\boldsymbol{\Sigma}_{y y}$.

In case the matrix $\boldsymbol{S}$ contains all source indices, we have $\boldsymbol{A}_{S}=\boldsymbol{A}_{\boldsymbol{S}_{J}}$. Then, $\left\|\left(\boldsymbol{I}-P_{\boldsymbol{A}_{\boldsymbol{S}_{J}}}\right) \boldsymbol{R}\right\|_{2}$, using (3.22), becomes

$$
\left\|\left(\boldsymbol{I}-P_{\boldsymbol{A}_{\boldsymbol{S}}}\right)\left(\boldsymbol{A}_{S} \hat{\boldsymbol{\Sigma}}_{s s} \boldsymbol{A}_{S}^{H}+\hat{\sigma}_{v}^{2} \boldsymbol{I}_{M}\right)\right\|_{2} .
$$

Putting $P_{\boldsymbol{A}_{\boldsymbol{S}}}=\boldsymbol{A}_{\boldsymbol{S}}\left(\boldsymbol{A}_{\boldsymbol{S}}^{H} \boldsymbol{A}_{\boldsymbol{S}}\right)^{-1} \boldsymbol{A}_{\boldsymbol{S}}^{H}$ in (3.23), we get

$$
\left\|\left(\boldsymbol{I}-\boldsymbol{A}_{\boldsymbol{S}}\left(\boldsymbol{A}_{\boldsymbol{S}}^{H} \boldsymbol{A}_{\boldsymbol{S}}\right)^{-1} \boldsymbol{A}_{\boldsymbol{S}}^{H}\right)\left(\boldsymbol{A}_{S} \hat{\boldsymbol{\Sigma}}_{s s} \boldsymbol{A}_{S}^{H}+\hat{\sigma}_{v}^{2} \boldsymbol{I}_{M}\right)\right\|_{2} .
$$


After simplifying, (3.24) becomes

$$
\hat{\sigma}_{v}^{2}\left\|\boldsymbol{I}-P_{\boldsymbol{A}_{S}}\right\|_{2}
$$

$\hat{\sigma}_{v}^{2}$ is an unknown parameter. Unlike previous case, we cannot approximate $\hat{\sigma}_{v}^{2}$ with $\lambda_{\boldsymbol{R}, \min }$, the minimum eigenvalue, which corresponds to the noise power. We should use some other technique to approximate the noise power. This technique is discussed in Section 3.3.3.

Putting (3.25) in (3.15), we get the value of $\mu$ :

$$
\hat{\sigma}_{v}^{2} \frac{\left\|\boldsymbol{I}-P_{\boldsymbol{A}_{\boldsymbol{S}_{J}}}\right\|_{2}}{\left\|\boldsymbol{I}-P_{\boldsymbol{A}_{\boldsymbol{S}_{J}}}\right\|_{2}}<\mu
$$

or,

$$
\hat{\sigma}_{v}^{2}<\mu
$$

Since the parameter $\mu$ is a thresholding parameter, we should set its value a bit higher than $\hat{\sigma}_{v}^{2}$.

\subsubsection{Bounds for the Optimization Parameter $\beta$}

In [31], Reddy et. al. have derived bounds for the value of $\beta$. These values depend on the lowest and highest eigenvalues of MVDR spectrum:

$$
\max _{\theta \in \boldsymbol{\Theta}} \frac{\lambda_{\boldsymbol{R}, \min }}{\left(\boldsymbol{a}_{\theta}^{H} \boldsymbol{R}^{-1} \boldsymbol{a}_{\theta}\right)^{-1}}<\beta<\min _{\theta \in \boldsymbol{\Theta}} \frac{\lambda_{\boldsymbol{R}, \min }}{\left(\boldsymbol{a}_{\theta}^{H} \boldsymbol{R}^{-1} \boldsymbol{a}_{\theta}\right)^{-1}}
$$

We need to calculate inverse for $\boldsymbol{R}$ matrix to be able to calculate bounds for $\beta$. $\boldsymbol{R}^{-1}$ for rank-deficient covariance matrix, $\boldsymbol{R}$, cannot be calculated. When we have $r<M, \operatorname{rank}(\boldsymbol{R})=r<M$, so matrix $\boldsymbol{R}$ in this case is not full rank. We calculate bounds of $\beta$ that are not dependent on $\boldsymbol{R}^{-1}$, as follows.

The solution to $(2.29)$ is given by $(2.30)$. 
Alternatively, we can write:

$$
\left\{\boldsymbol{a}_{\theta} \boldsymbol{a}_{\theta}^{H}+\beta \boldsymbol{I}\right\}^{-1} \boldsymbol{R} \boldsymbol{w}_{\theta}=\lambda_{\min } \boldsymbol{w}_{\theta}
$$

By using Woodbury identity, we can write $\left\{\boldsymbol{a}_{\theta} \boldsymbol{a}_{\theta}^{H}+\beta \boldsymbol{I}\right\}^{-1}$ as

$$
\frac{\boldsymbol{I}}{\beta}+\frac{\boldsymbol{a}_{\theta} \boldsymbol{a}_{\theta}^{H}}{\beta}-\frac{\boldsymbol{a}_{\theta} \boldsymbol{a}_{\theta}^{H}}{\beta+1},
$$

inserting (3.30) into (3.29), we get

$$
\left\{\frac{\boldsymbol{I}}{\beta}+\frac{\boldsymbol{a}_{\theta} \boldsymbol{a}_{\theta}^{H}}{\beta}-\frac{\boldsymbol{a}_{\theta} \boldsymbol{a}_{\theta}^{H}}{\beta+1}\right\} \boldsymbol{R} \boldsymbol{w}_{\theta}=\lambda_{\min } \boldsymbol{w}_{\theta} .
$$

Pre-multiplying both sides by $\boldsymbol{w}_{\theta}^{H}$, we get

$$
\frac{\boldsymbol{w}_{\theta}^{H}\left\{\frac{\boldsymbol{R}}{\beta}+\frac{\boldsymbol{\Psi}}{\beta}-\frac{\boldsymbol{\Psi}}{\beta+1)}\right\} \boldsymbol{w}_{\theta}}{\left\|\boldsymbol{w}_{\theta}\right\|_{2}^{2}}=\lambda_{\min }, \quad \boldsymbol{\Psi}:=\boldsymbol{a}_{\theta} \boldsymbol{a}_{\theta}^{H} \boldsymbol{R} .
$$

\section{When the Steering Vector $a_{j}$ Lies Inside the Signal Subspace}

The solution $\lambda_{\min }$ is given by the smallest eigenvalue of $\left\{\frac{\boldsymbol{R}}{\beta}+\frac{\boldsymbol{\Psi}}{\beta}-\frac{\boldsymbol{\Psi}}{\beta+1}\right\}$. As $\boldsymbol{w}^{H} \boldsymbol{a}_{j}=$ 0 , if $j \in \operatorname{supp} \boldsymbol{X}$, then $\boldsymbol{w}_{\theta}^{H} \boldsymbol{\Psi}=0$, and we can write

$$
\frac{\boldsymbol{w}_{\theta}^{H}\left\{\frac{\boldsymbol{R}}{\beta}+\frac{\boldsymbol{\Psi}}{\beta}-\frac{\boldsymbol{\Psi}}{\beta+1}\right\} \boldsymbol{w}_{\theta}}{\left\|\boldsymbol{w}_{\theta}\right\|_{2}^{2}} \approx \frac{\boldsymbol{w}_{\theta}^{H} \boldsymbol{R} \boldsymbol{w}_{\theta}}{\beta\left\|\boldsymbol{w}_{\theta}\right\|_{2}^{2}}
$$

This condition holds, when

$$
\frac{\boldsymbol{w}_{\theta}^{H} \boldsymbol{R} \boldsymbol{w}_{\theta}}{\beta\left\|\boldsymbol{w}_{\theta}\right\|_{2}^{2}} \gg \frac{\boldsymbol{w}_{\theta}^{H} \boldsymbol{\Psi} \boldsymbol{w}_{\theta}}{\beta\left\|\boldsymbol{w}_{\theta}\right\|_{2}^{2}}
$$

and

$$
\frac{\boldsymbol{w}_{\theta}^{H} \boldsymbol{R} \boldsymbol{w}_{\theta}}{\beta\left\|\boldsymbol{w}_{\theta}\right\|_{2}^{2}} \gg \frac{\boldsymbol{w}_{\theta}^{H} \boldsymbol{\Psi} \boldsymbol{w}_{\theta}}{(\beta+1)\left\|\boldsymbol{w}_{\theta}\right\|_{2}^{2}} .
$$

Since $\boldsymbol{a}_{\theta} \boldsymbol{a}_{\theta}^{H}$ is rank one matrix. Multiplying this matrix with any other matrix would yield rank one matrix. Therefore, $\boldsymbol{\Psi}$ is also rank one matrix. The maximum 
eigenvalue $\lambda_{\boldsymbol{\Psi}, \max }=\operatorname{tr}(\boldsymbol{\Psi})$. The R.H.S of (3.34) goes to zero as $\boldsymbol{w}^{H} \boldsymbol{a}_{j}=0$, if $j \in \operatorname{supp} \boldsymbol{X}$. So we can only say that the minimum eigenvalue of $\boldsymbol{R}$ should be greater than zero. That is,

$$
\lambda_{R, \min }>0 .
$$

As $\boldsymbol{R}$ is a positive definite matrix, this is certainly true.

Using identity $\operatorname{tr}(\boldsymbol{E} \boldsymbol{F} \boldsymbol{G})=\operatorname{tr}(\boldsymbol{F G} \boldsymbol{E})$, we rewrite (3.35), to highlight the highest bound of R.H.S.:

$$
\frac{\boldsymbol{w}_{\theta}^{H} \boldsymbol{R} \boldsymbol{w}_{\theta}}{\beta\left\|\boldsymbol{w}_{\theta}\right\|_{2}^{2}} \gg \max _{\theta \in \operatorname{supp} \boldsymbol{X}} \frac{\operatorname{tr}\left(\boldsymbol{a}_{\theta} \boldsymbol{a}_{\theta}^{H} \boldsymbol{R}\right)}{\beta+1} .
$$

Rearranging the argument of $\operatorname{tr}($.$) and scalarizing it, we get$

$$
\frac{\boldsymbol{w}_{\theta}^{H} \boldsymbol{R} \boldsymbol{w}_{\theta}}{\beta\left\|\boldsymbol{w}_{\theta}\right\|_{2}^{2}} \gg \max _{\theta \in \text { supp } \boldsymbol{X}} \frac{\boldsymbol{a}_{\theta}^{H} \boldsymbol{R} \boldsymbol{a}_{\theta}}{\beta+1} .
$$

The L.H.S can be further simplified by using the approximation in (3.33) and the value of $\frac{\boldsymbol{w}_{\theta}^{H} \boldsymbol{R} \boldsymbol{w}_{\theta}}{\left\|\boldsymbol{w}_{\theta}\right\|_{2}^{2}}=\lambda_{\boldsymbol{R}, \min }$. Therefore,

$$
\frac{\lambda_{\boldsymbol{R}, \min }}{\beta} \gg \max _{\theta \in \sup \boldsymbol{X}} \frac{\boldsymbol{a}_{\theta}^{H} \boldsymbol{R} \boldsymbol{a}_{\theta}}{\beta+1} .
$$

Simplifying further, we can get the lowest bound for $\beta$

$$
\beta>\frac{\lambda_{\boldsymbol{R}, \min }}{\max _{\theta \in \operatorname{supp} \boldsymbol{X}}\left\{\boldsymbol{a}_{\theta}^{H} \boldsymbol{R} \boldsymbol{a}_{\theta}\right\}-\lambda_{\boldsymbol{R}, \min }} .
$$

Since $\left\{\frac{R}{\beta}+\frac{\Psi}{\beta}-\frac{\Psi}{\beta+1}\right\}$ is positive definite (it is a product of two positive definite matrices), we expect $\lambda_{\min }$ to be positive as well.

If the following conditions are true, the matrix would remain positive definite otherwise it can get negative. So, the following two inequalities needs to be obeyed:

$$
\frac{\boldsymbol{w}_{\theta}^{H} \boldsymbol{\Psi} \boldsymbol{w}_{\theta}}{\beta\left\|\boldsymbol{w}_{\theta}\right\|_{2}^{2}}-\frac{\boldsymbol{w}_{\theta}^{H} \boldsymbol{\Psi} \boldsymbol{w}_{\theta}}{(\beta+1)\left\|\boldsymbol{w}_{\theta}\right\|_{2}^{2}} \geq 0
$$


and

$$
\frac{\boldsymbol{w}_{\theta}^{H} \boldsymbol{R} \boldsymbol{w}_{\theta}}{\beta\left\|\boldsymbol{w}_{\theta}\right\|_{2}^{2}}>\frac{\boldsymbol{w}_{\theta}^{H} \boldsymbol{\Psi} \boldsymbol{w}_{\theta}}{(\beta+1)\left\|\boldsymbol{w}_{\theta}\right\|_{2}^{2}} .
$$

By simplifying (3.41), we get

$$
\min _{\theta \notin \operatorname{supp} \boldsymbol{X}}\left\{\boldsymbol{a}_{\theta}^{H} \boldsymbol{R} \boldsymbol{a}_{\theta}\right\} \geq 0
$$

Since, $\boldsymbol{R}$ is a positive definite matrix, there is no $\boldsymbol{a}_{\theta}$ that would violate (3.43).

\section{When the Steering Vector $a_{j}$ does not Belong to the Signal Subspace}

For $\boldsymbol{a}_{j}$ when $j \notin \operatorname{supp} \boldsymbol{X}$, it is important to have an upper bound on the value of $\beta$ so that we do not have spurious peaks. We should limit the highest value of $\beta$, as to not make the L.H.S. greater than the R.H.S. of (3.42.) Reversing the inequality in (3.42), we can find the upper bound of $\beta$ with the following inequality:

$$
\frac{\boldsymbol{w}_{\theta}^{H} \boldsymbol{R} \boldsymbol{w}_{\theta}}{\beta\left\|\boldsymbol{w}_{\theta}\right\|_{2}^{2}}<\frac{\boldsymbol{w}_{\theta}^{H} \boldsymbol{\Psi} \boldsymbol{w}_{\theta}}{(\beta+1)\left\|\boldsymbol{w}_{\theta}\right\|_{2}^{2}} .
$$

Using $\frac{\boldsymbol{w}_{\theta}^{H} \boldsymbol{R} \boldsymbol{w}_{\theta}}{\left\|\boldsymbol{w}_{\theta}\right\|_{2}^{2}}=\lambda_{\boldsymbol{R}, \text { min }}$ and setting the lowest value for R.H.S of (3.44), we can get the highest bound for $\beta$ :

$$
\beta<\frac{\lambda_{\boldsymbol{R}, \min }}{\min _{\theta \notin \operatorname{supp} \boldsymbol{X}}\left\{\boldsymbol{a}_{\theta}^{H} \boldsymbol{R} \boldsymbol{a}_{\theta}\right\}-\lambda_{\boldsymbol{R}, \min }} .
$$

Therefore, the bounds for $\beta$ is given by

$$
\frac{\lambda_{\boldsymbol{R}, \min }}{\xi_{\max }-\lambda_{\boldsymbol{R}, \min }}<\beta<\frac{\lambda_{\boldsymbol{R}, \min }}{\xi_{\min }-\lambda_{\boldsymbol{R}, \min }}
$$

where, $\xi_{\max }=\max _{\theta \in \sup \boldsymbol{X}}\left\{\boldsymbol{a}_{\theta}^{H} \boldsymbol{R} \boldsymbol{a}_{\theta}\right\}$ and $\xi_{\min }=\underset{\theta \notin \min \boldsymbol{X}}{\min }\left\{\boldsymbol{a}_{\theta}^{H} \boldsymbol{R} \boldsymbol{a}_{\theta}\right\}$. 


\subsubsection{When the Approximation $R \approx \Sigma_{y y}$ Becomes Invalid Because of Very Few Snapshots}

These bounds work under approximation that $\boldsymbol{R} \approx \boldsymbol{\Sigma}_{y y}$. This approximation is valid when we have a high number of snapshots. The only problem when $\boldsymbol{R}$ matrix is rank deficient is that we would be unable to calculate $\lambda_{\boldsymbol{R}, \min }$. But we know that $\lambda_{\boldsymbol{R}, \min }=\sigma_{v}^{2}$ when the covariance matrix is full rank. That means we have to calculate $\sigma_{v}^{2}$, using method other than eigenvalue decomposition of $\boldsymbol{R}$.

We propose to using beamformer spectrum using Fast Fourier Transform (FFT) to calculate $\sigma_{v}^{2}$. Below are the steps that are required to estimate $\sigma_{v}^{2}$.

i For the first step, we sum and normalize all the snapshots: $\hat{\boldsymbol{y}}=\frac{1}{r} \sum_{i=1}^{r} \frac{\boldsymbol{y}_{i}}{\left\|\boldsymbol{y}_{i}\right\|_{2}}$.

ii We compute beamformer output for all directions in the following manner: $B F(\theta)=\boldsymbol{a}_{\theta}^{H} \hat{\boldsymbol{y}}$.

iii We compute power spectrum using Fourier transform: $B F(\theta) \stackrel{\mathscr{F}}{\rightarrow} B F(\omega)$ and sort it in ascending order, $\hat{B F}(\omega)=\operatorname{sort}\{B F(\omega)\}$.

iv $\hat{\sigma}_{v}^{2}=\frac{1}{J} \sum_{i=1}^{J} B \hat{F}(\omega) . J$ is an arbitrary number. Its value can be set to 64,128 or 256 .

These bounds, do not contain the term $\boldsymbol{R}^{-1}$ and work for rank deficient covariance matrices. So, these bounds are much more general than those derived in [31]. 


\subsection{Results}

In this section, we demonstrate the performance of the recursive MUSIC-like algorithm in comparison to a well known MMV algorithm: Simultaneous Orthogonal Matching Pursuit (SOMP) [16]. The benefits of Recursive MUSIC-like Algorithm over conventional MMV algorithms is the integration capability of the multiple measurement vectors through the use of covariance matrix. The covariance matrix is computed in the following manner:

$$
\boldsymbol{R}=\frac{1}{r} \sum_{i=1}^{r} \boldsymbol{y}_{i} \boldsymbol{y}_{i}^{T} .
$$

Here, $r$ is the number of snapshots or the number of data vectors, $\boldsymbol{y}$. As the number of snapshots increase, the computed covariance matrix, $\boldsymbol{R}$, starts to approach the true data covariance matrix, $\Sigma_{y y}$, given by

$$
\boldsymbol{\Sigma}_{y y}=\boldsymbol{A}_{S} \boldsymbol{\Sigma}_{s s} \boldsymbol{A}_{S}^{H}+\sigma_{v}^{2} \boldsymbol{I}_{M}
$$

Where, $\boldsymbol{\Sigma}_{s s}$ is the true source covariance matrix.

Figure 3.4 shows the stoppage function for two cases. The first case is when we have full rank covariance matrix, formed using 100 snapshots. $\mu$ is calculated using minimum eigenvalue $\lambda_{\boldsymbol{R} \text {,min }}$ of computed covariance matrix, $\boldsymbol{R}$. The second case is when we have rank deficient matrix, with $\mathrm{r}=4$ and number of sources, $K=6$. In this case $\lambda_{\boldsymbol{R}, \min }$ is not available so we use power spectrum, using the Fourier transform, to calculate the value of $\mu$. In both cases the correct number of sources $(\mathrm{K}=6)$ have been identified.

There are two important dimensions of the CS MMV problem discussed in this chapter. The first one is the number of sources vs the number of sensors. The second aspect is the effect of the number of measurement vectors/snapshots on the 


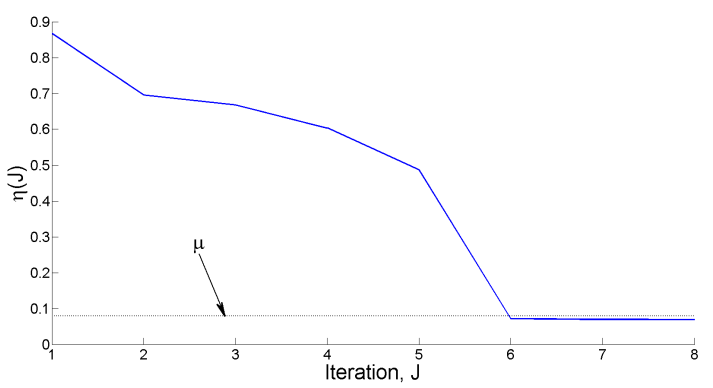

(a)

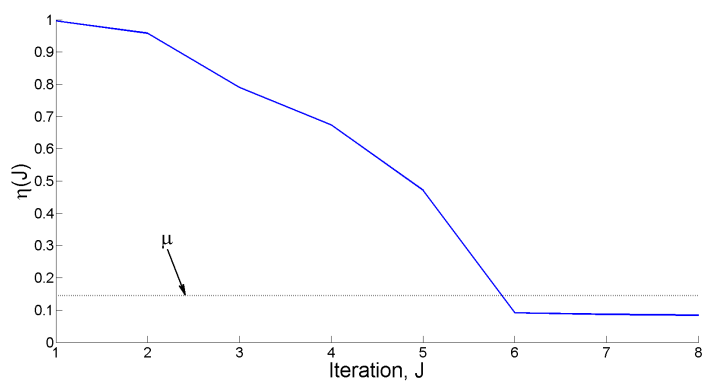

(b)

Figure 3.4: Stopping function $\eta(J)$ shown for $\mathrm{M}=31, \mathrm{~K}=6$ and $\mathrm{SNR}=0 \mathrm{~dB}$, when the covariance matrix is a) full rank $(\mathrm{r}=100)$, b) rank deficient $(\mathrm{r}=4)$. 


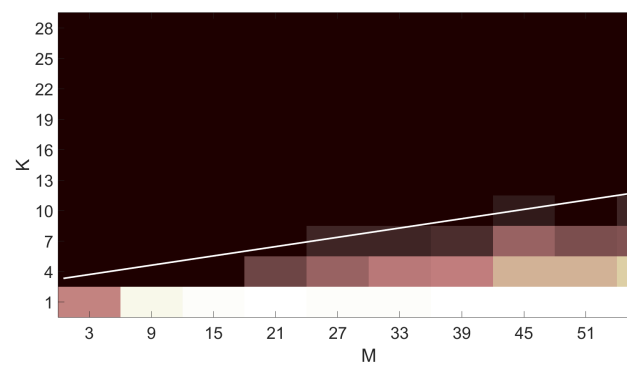

(a)

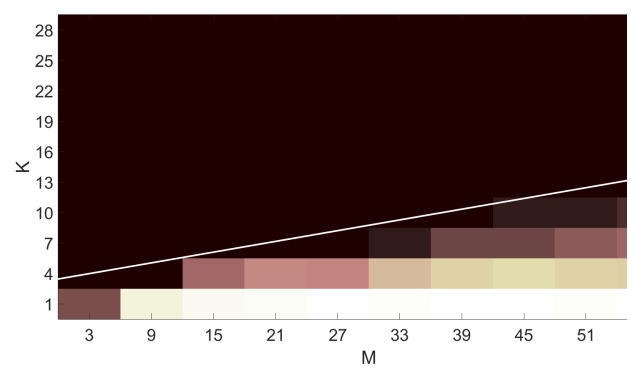

(c)

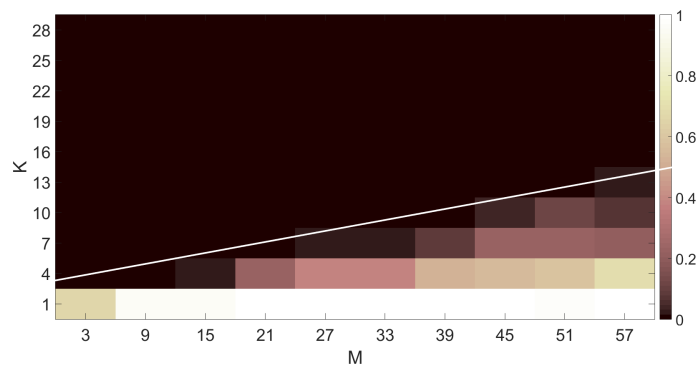

(b)

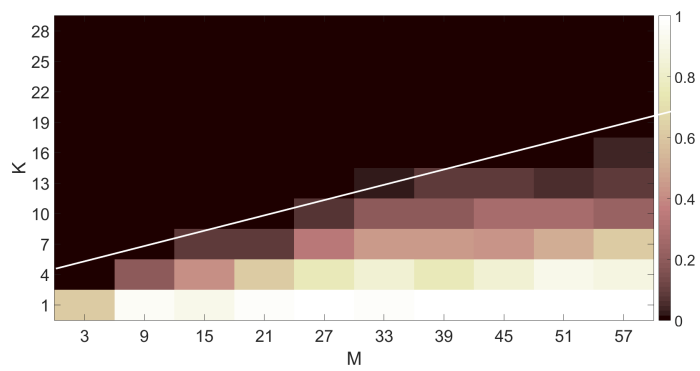

(d)

Figure 3.5: Recovery rate maps for different values of $\mathrm{M}$ and $\mathrm{K}$ when $\mathrm{SNR}=20 \mathrm{~dB}$ and $\mathrm{N}=180$, the white line defines the boundary between the recovery region and the non-recovery region, a) Recovery rate map for SOMP when $\mathrm{r}=4$, b) Recovery rate map for SOMP when $r=16, c)$ Recovery rate map for $R M L$ when $r=4, d$ ) Recovery rate map for RML when $\mathrm{r}=16$.

performance of the CS MMV algorithms. Figure 3.5 shows the performance of the SOMP and RML algorithms from the first aspect where the number of snapshots are held constant while we study the number of sources that can be successfully recovered by using the two algorithms. In case of Figure 3.6, we study the effect of increase in the number of snapshots and the ability of recovery of the two algorithms 
when both the number of sources and and number of sensors are kept constant.

We compare the two algorithms (RML and SOMP) for different values of $\mathrm{M}, \mathrm{K}$ and $\mathrm{r}: M \in\{3,9, \ldots, 57\}, K \in\{1,4, \ldots, 28\}, r \in\{4,16\}$ and $N=180$. The $\mathrm{SNR}$ is set to $20 \mathrm{~dB}$. The detection was regarded as successful, when all of the sources in $\operatorname{supp} \boldsymbol{X}$ were correctly detected. The sources were generated randomly. The matrix $\boldsymbol{A}$ was generated using random sensor positions. This was done to achieve low mutual coherence. The success rate/recovery rate was averaged over 200 montecarlo experiments. Figure 3.5 shows the recovery rate of the two algorithms SOMP and RML. White color shows successful detection in all cases, black color indicates that the algorithms failed to detect all the sources (this requirement is stringent as compared to probability of resolution), while the different shades of gray indicate different recovery rates. As we can see the detection for SOMP does not vary much when $\mathrm{r}=4$ from the case when $\mathrm{r}=16$. While for $\mathrm{r}=4$, RML recovery rate is comparable to SOMP, but when we increase the number of snapshots to $\mathrm{r}=16$, RML shows clear advantage over SOMP. Both the scenarios $(\mathrm{r}=4$ and $\mathrm{r}=16$ ) would fall under compressive sensing paradigm, when $r<M$. For the case where we have a very few $\operatorname{snapshots}(r=4)$, the performance of both SOMP and RML is comparable. For the case when the snapshots are increased $(r=16)$, RML performs significantly better than SOMP. For instance, when $M=39$, this would fall in the compressed sensing scenario as we have only $r=16$ snapshots, RML successfully recovered, at maximum $M=15$ sources, while for the same parameters SOMP successfully recovered $M=8$ sources, which shows the advantage of RML over SOMP.

The term probability of resolution $\left(P_{R}\right)$ is defined for resolution of two sources in [1]. We have used the same definition when finding two sources. When we have more than two sources then the term probability of resolution is extended in the 


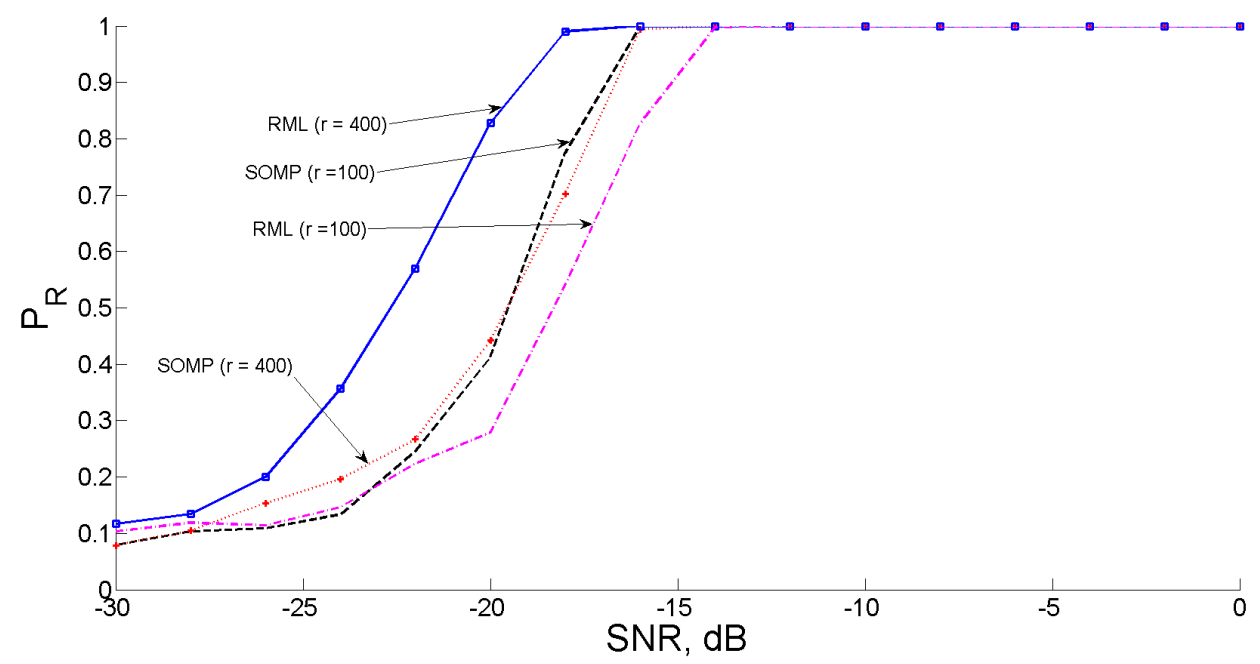

Figure 3.6: $P_{R}$ vs SNR curves for RML and SOMP algorithm for values of $\mathrm{M}=39$ and $\mathrm{K}=9$.

following manner. Suppose $M$ sources are located at $u_{1}, u_{2}, \cdots, u_{M}$. We define the boundary between the adjacent source pairs $(u 1, u 2), \quad(u 2, u 3), \cdots, \quad\left(u_{M-1}, u_{M}\right)$ as $\hat{u}_{1}, \cdots, \hat{u}_{M-1}$, where $\hat{u}_{m}=\frac{u_{m}-u_{m-1}}{2}$. The minimum bound for the first source is $\hat{u}_{0}=u_{1}-\delta$, while the maximum bound for the last source is $\hat{u}_{0}=u_{M}+\delta$. $\delta$ is a small number. In our simulations we have used $\delta=1$. The sources are considered resolved if all the $\mathrm{M}$ peaks lie within the boundaries defined.

The effects of increase in the number of snapshots for both SOMP and RML is clear from Figure 3.6. The figure shows the probability of resolution of sources when $\mathrm{M}=39$ and $\mathrm{K}=9$, for different value of SNR. The values were averaged over 200 trials. We can see that the SOMP algorithm shows no improvement in detection when we increase the number of snapshots significantly (from 100 to 400). While, in case of RML, when the snapshots are increased, we see clear increase in the probability of resolution, $P_{R}$, of the sources. 


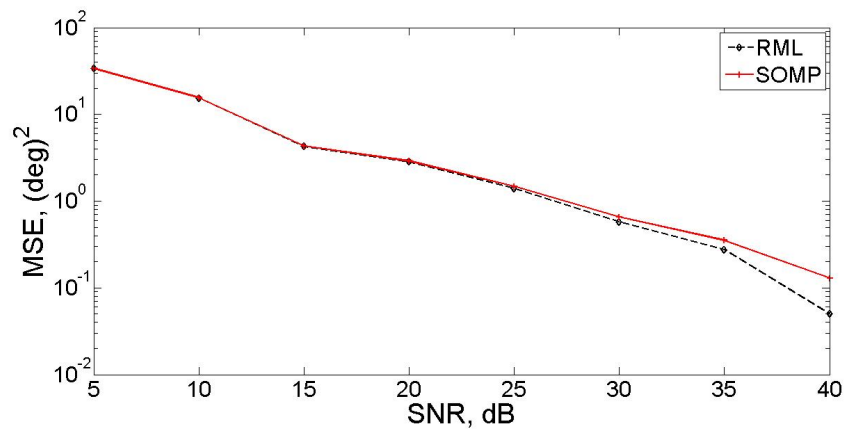

(a)

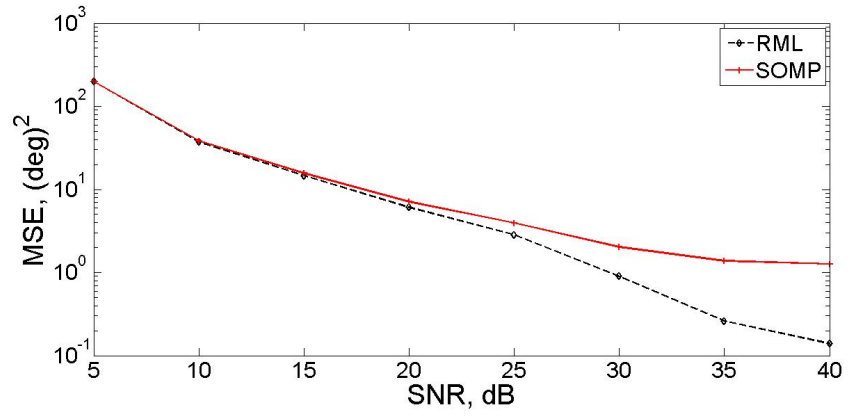

(b)

Figure 3.7: MSE for two sources plotted against SNR for RML and SOMP for $r=10, M=11$ and a) a source at 37 degrees, b) a source at 43 degrees. 


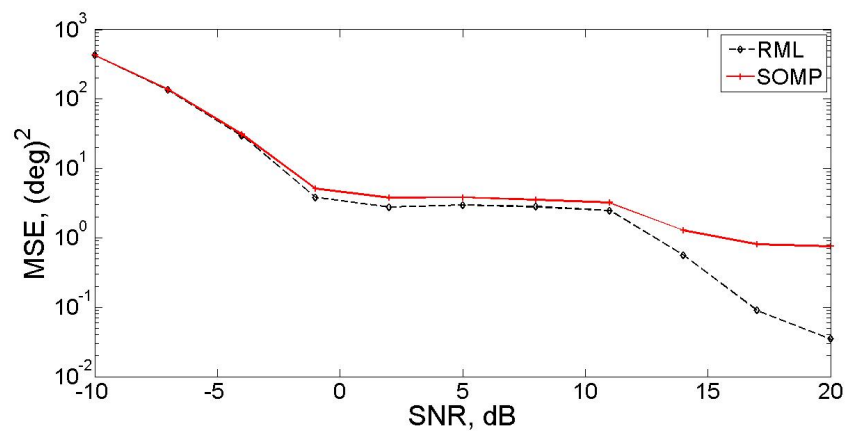

(a)

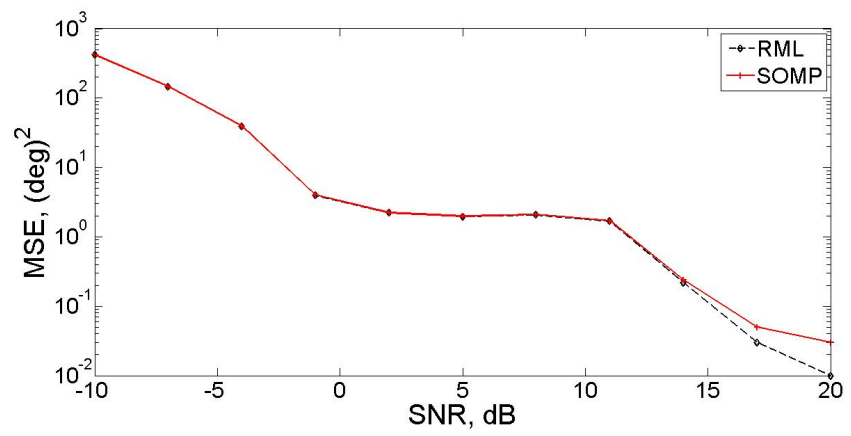

(b)

Figure 3.8: MSE for two sources plotted against SNR for RML and SOMP for $r=100, M=11$ and a) a source at 35 degrees, b) a source at 39 degrees. Note that the horizontal axis has been shifted left for this figure as compared to Figure 3.7 as there are more snapshots available. 
Figure 3.7 shows a plot of Mean Squared Error (MSE) for two sources at 37 and 43 degrees for both the RML and SOMP algorithms for 200 Montecarlo simulations. Where, MSE has been calculated using the following equation:

$$
M S E=\frac{1}{k} \sum_{i=1}^{k}\left(x_{i}-\hat{x}_{i}\right)^{2} .
$$

Here, $\hat{\boldsymbol{x}}$ is the estimate of $\boldsymbol{x}$. While, $\boldsymbol{x}$ is a vector containing true source directions. The number of snapshots are very few $(r=10)$. It is clear from the figure that the MSE for RML algorithm decreases at a faster rate for both the sources as compared to SOMP algorithm and is always lower than that of SOMP algorithm. Similarly, Figure 3.8 shows a plot of Mean Squared Error (MSE) for two sources at 35 and 49 degrees (6 degrees separation) for both the RML and SOMP algorithms for 200 Montecarlo simulations. The angular separation between the sources is 4 degrees as compared to the previous case when separation was kept at 6 degrees. The number of snapshots are significantly more $(r=100)$. For source 1 the difference is that the MSE is much higher as compared to that of source 2, but the RML algorithm, for all values of SNR, performs either equally as good or better than the SOMP algorithm. As we are using more snapshots, the algorithms can perform much better in this case, so the SNR range for this figure is lower than that of Fig 3.7.

We have studied two important properties in this section. Firstly, we demonstrated the ability of RML algorithm to recover sources from noisy data when we have a very few number of sources. Lastly, we showed that when the number of snapshots increases, the ability of RML algorithm to detect sources in noisy environment also improves. 


\subsection{Conclusions}

In this Chapter, we have developed a criterion similar to the generalized MUSIC criterion for the MUSIC-like algorithm. This criterion, similar to the MUSIC-like algorithm, does not depend on the knowledge of the number of sources. We extended the GMLC to devise an MMV compressive sensing algorithm that does not presuppose any knowledge of the number of sources. RML, being a covariance matrix based algorithm, shows clear advantage over SOMP algorithm when we increase the number of snapshots. We, derived lower and upper bounds for parameter $\beta$ for degenerate covariance matrix, which is essential to the working of MUSIC-like algorithm. This made it possible to derive a Recursive MUSIC-like algorithm that does not depend on the inversion of the covariance matrix, which is singular when $r<K$ 


\section{Chapter 4}

\section{Recursive MUSIC-Like Algorithm}

\section{for DOA Estimation of Broadband}

\section{Sources}

\subsection{Introduction}

In Chapter 3, we developed a recursive algorithm, to estimate direction of arrival of multiple sources, in a compressive sensing scenario. Here, we seek to extend the same idea to the direction of arrival estimation, when the sources are emitting broadband signals, instead of narrowband signals, as was the case before. Several algorithms exist that tackle this problem for Nyquist sampling case. Most of them try to do some kind of frequency focusing to align steering vectors from different frequencies and remove frequency dependence from them. One of the schemes using Taylor Series Expansion (TSE) to remove the effects of higher order derivatives from 
the steering vector [35]. Compressed sensing, is a relatively new field of research. From a linear algebra perspective, we can see the steering vectors as basis for a vector space. These basis vectors have to be as uncorrelated or linearly independent as possible, for the CS algorithms to work reliably. Because of the frequency dependence of the steering vector, this linear-independence or orthogonality is compromised. So, dealing with a matrix that has this frequency dependence does not produce good results.

We will deal with this issue of linear dependence in several ways. The most obvious way is to use focusing matrices to focus the steering vectors and then apply Compressive Sensing algorithms subsequently. We study this approach in Section 4.2.2. Another way is to use TSE to remove effects of high order derivatives form the steering vectors. This approach is discussed in Section 4.2.1. But the actual algorithm used to detect the sources is not straightforward. MUSIC or MVDR algorithms are usually used to estimate the direction of arrival of the broadband sources. The CS counterparts are $l_{1}$ minimization approach and several forms of greedy algorithms. $l_{1}$ minimization is very sensitive to basis variations. So we are left with SOMP algorithm to address this issue. In Chapter 3, we developed a new algorithm, RML, that addresses this problem. It is a hybrid of the classical and Compressive Sensing approaches. We, in this case use SOMP and RML to address the DOA estimation problem for broadband sources.

\subsection{Broadband Approaches to DOA Estimation}

For the broadband signals, the steering vector is quite different from the narrowband case, as it is dependent on both the frequency as well as the direction of arrival. 
This frequency dependence render the usage of narrowband approaches like MUSIC useless when we have low signal-to-noise ratio.

The signal model is given by

$$
\boldsymbol{y}(f)=\boldsymbol{A}(f, \Theta) \boldsymbol{x}(f)+\boldsymbol{n}(f) .
$$

$\boldsymbol{y}(f), \boldsymbol{n}(f) \in \mathbb{C}^{M \times 1}$ are sensor outputs converted to frequency-domain and additive noise vectors respectively. $\boldsymbol{s}(f)$ is the vector containing signals originating from $K$ sources. $\boldsymbol{A}(f, \Theta)$ is the frequency dependent array manifold matrix. The array manifold matrix consists of $K$ steering vectors corresponding to each source. The steering vector as a function of $f$ and $\theta$ is given below:

$$
\boldsymbol{a}(f, \theta)=e^{j \boldsymbol{\kappa}_{\theta}^{T} \boldsymbol{L}}=\frac{1}{\sqrt{m}}\left[e^{j \boldsymbol{\kappa}_{\theta}^{T} \boldsymbol{l}_{1}}, \ldots, e^{j \boldsymbol{\kappa}_{\theta}^{T} \boldsymbol{l}_{M}}\right]^{T},
$$

where $\boldsymbol{l}_{i}$ is the $i^{t h}$ sensor position vector and $\boldsymbol{\kappa}_{\theta}=\frac{2 \pi f}{\nu} \boldsymbol{v}_{\theta_{s}} . \boldsymbol{v}_{\theta_{s}}$ is the unit vector in the direction of source $s$. For 2-D Cartesian coordinates it is given by:

$$
\boldsymbol{v}_{\theta_{s}}=\left(\cos \theta_{s}, \sin \theta_{s}\right)
$$

In case of 3-D Cartesian coordinates we have an additional parameter $\phi$ denoting the polar angle. The direction unit vector in that case is given by:

$$
\boldsymbol{v}_{\theta_{s}}=\left(\cos \theta_{s} \sin \phi_{s}, \sin \theta_{s} \sin \phi_{s}, \cos \phi_{s}\right),
$$

where $\theta_{s} \in(0,2 \pi]$ and $\phi_{s} \in[0, \pi]$. The problem of the direction of arrival estimation is actually estimation of these parameters; azimuth in the case of 2-D Cartesian coordinates or linear array, azimuth and the polar angle (or elevation [Appendix A]) in the case of 3-D Cartesian coordinates and planar array. The Coordinate system used in the thesis is described in Appendix A. The geometry is dictated by the arrangement of sensor array elements. If the array elements form a curve we can only estimate one parameter. In case the sensor elements span a 2-D plane, however irregular, we can estimate both the parameters (azimuth and elevation). 


\subsubsection{Taylor Series Expansion Model}

In this model the frequency dependent steering vector is expanded using Taylor Series expansion (TSE) at a certain frequency $f_{0}$. The aim is to minimize the contribution of the higher order terms in the TSE. The expansion is given by:

$$
\boldsymbol{a}(f, \theta) \approx \boldsymbol{a}\left(f_{0}, \theta\right)+\left.\sum_{\xi=1}^{J} \frac{(\delta f)^{\xi}}{\xi !} \frac{\partial^{\xi} \boldsymbol{a}(f, \theta)}{\partial f^{\xi}}\right|_{f=f_{0}}
$$

This approximation is valid for derivative order $J$. As $J$ is increased the approximation becomes closer to $\boldsymbol{a}(f, \theta)$. The MUSIC-like algorithm is based on covariance matrix approach. We will have to see the effect of the derivative terms on the covariance matrix. Although the covariance matrix can be computed using either time domain snapshots or frequency domain snapshots, here we will only discuss the covariance matrix calculated through frequency domain snapshots, as this matrix is the one that we will be using in our algorithms. The array manifold matrix can be expanded using TSE as follows:

$$
\boldsymbol{A}(f, \theta) \approx \boldsymbol{A}\left(f_{0}, \Theta\right)+\left.\sum_{\xi=1}^{J} \frac{(\delta f)^{\xi}}{\xi !} \frac{\partial^{\xi} \boldsymbol{A}(f, \theta)}{\partial f^{\xi}}\right|_{f=f_{0}}
$$

Putting the TSE of $\boldsymbol{A}(f, \theta)$ in (4.1), we get:

$$
\boldsymbol{y}(f) \approx\left(\boldsymbol{A}\left(f_{0}, \Theta\right)+\left.\sum_{\xi=1}^{J} \frac{(\delta f)^{\xi}}{\xi !} \frac{\partial^{\xi} \boldsymbol{A}(f, \theta)}{\partial f^{\xi}}\right|_{f=f_{0}}\right) \boldsymbol{x}(f)+\boldsymbol{n}(f) .
$$

The frequency dependent covariance matrix, $R(f)$, becomes

$$
\begin{aligned}
\boldsymbol{R}_{y y}(f) \quad \approx \boldsymbol{A}\left(f_{0}, \Theta\right) \boldsymbol{R}_{x x}(f) \boldsymbol{A}\left(f_{0}, \Theta\right)^{H}+\sigma_{n}^{2}(f) \boldsymbol{I}_{M} \\
+\sum_{\xi=1}^{J} \frac{\delta f^{\xi}}{\xi !} \mathfrak{R}\left(\left.\boldsymbol{A}\left(f_{0}, \Theta\right) \boldsymbol{R}_{x x}(f) \frac{\partial^{\xi}}{\partial f^{\xi}} \boldsymbol{A}(f, \Theta)^{H}\right|_{f=f_{0}}\right) \\
+\left.\left.\sum_{\xi, \zeta=1}^{J} \frac{\delta f^{\xi \zeta}}{\xi ! \zeta !} \frac{\delta f^{\xi}}{\xi !} \boldsymbol{A}(f, \Theta)\right|_{f=f_{0}} \boldsymbol{R}_{x x}(f) \frac{\partial^{\xi}}{\partial f^{\xi}} \boldsymbol{A}(f, \Theta)^{H}\right|_{f=f_{0}}
\end{aligned}
$$


here, $\boldsymbol{R}_{x x}(f)$ is the source covariance matrix, $\sigma_{n}^{2}(f)$ is the variance of noise at each frequency $f$. $\boldsymbol{I}_{M}$ denotes an identity matrix of dimension $M \times M$. The matrix $\sigma_{n}^{2}(f) \boldsymbol{I}_{M}$ denotes the sensor noise covariance matrix. Since the noise is uncorrelated across different sensors, therefore, we have a diagonal matrix. The first term in (4.9) contains array manifold matrix at $f_{0}$, the second term contains second order noise statistics, the rest of the terms are clearly dependent on higher order derivatives. This shows why the narrowband approaches using covariance matrix does not work. Reddy et. al. [35] have suggested to construct covariance matrix using the frequency bins that contain the source frequency. The out of band frequency bins are simply discarded. In Section 2.4.4 we have seen the formulation of MUSIC-like algorithm. Reddy et. al. [35] suggested to use a modified covariance matrix $\boldsymbol{R}_{\boldsymbol{t}}$ instead of the original matrix $\boldsymbol{R}$ used in the narrowband formulation (2.29). The broadband formulation as suggested in [35] is as follows:

$$
\begin{aligned}
\underset{\boldsymbol{w}_{\theta}}{\operatorname{minimize}} & \boldsymbol{w}_{\theta}^{H} \boldsymbol{R}_{t} \boldsymbol{w}_{\theta}, \\
\text { s.t. } & \boldsymbol{w}_{\theta}^{H}\left\{\boldsymbol{a}_{\theta} \boldsymbol{a}_{\theta}^{H}+\beta \boldsymbol{I}\right\} \boldsymbol{w}_{\theta}=c,
\end{aligned}
$$

where $c, \beta>0$ are constants. Its solution is found by solving the following generalized eigenvalue problem:

$$
\boldsymbol{R}_{t} \boldsymbol{w}_{\theta}=\lambda_{\min }\left\{\boldsymbol{a}_{\theta} \boldsymbol{a}_{\theta}^{H}+\beta \boldsymbol{I}\right\} \boldsymbol{w}_{\theta}, \theta \in(0, \pi]
$$

The solution weight vector, $\boldsymbol{w}_{\theta}$, for each direction, $\theta$, is the eigenvector corresponding to the minimum eigenvalue of (4.10). Matrix $\boldsymbol{R}_{t}$ is given by:

$$
\boldsymbol{R}_{t}=\boldsymbol{R}_{a v}+\chi \boldsymbol{R}_{y}(\theta)
$$

where, $\boldsymbol{R}_{a v}$ and $\boldsymbol{R}_{y}$ matrices are calculated in the following manner:

$$
\boldsymbol{R}_{a v}=\frac{1}{D+1} \sum_{i=1}^{D+1} \boldsymbol{R}\left(f_{i}\right)
$$




$$
\boldsymbol{R}_{y}(\theta)=P_{\boldsymbol{D}_{\theta}} \boldsymbol{R}_{a v} P_{\boldsymbol{D}_{\theta}}^{H} .
$$

Here $P_{(.)}$represents orthogonal projection matrix on to the subspace defined by columns of a matrix. For a given direction $\theta$, the derivative manifold matrix, $\boldsymbol{D}_{\theta}$, is defined as:

$$
\boldsymbol{D}_{f_{0}}(\theta)=\left[\begin{array}{llll}
\boldsymbol{d}_{1}\left(f_{0}, \theta\right) & \boldsymbol{d}_{2}\left(f_{0}, \theta\right) \quad \ldots \quad \boldsymbol{d}_{J}\left(f_{0}, \theta\right)
\end{array}\right]
$$

Here, $\boldsymbol{d}_{1}\left(f_{0}, \theta\right)=\frac{\partial^{j} \boldsymbol{a}\left(f_{0}, \theta\right)}{\partial f^{j}}$. $J$ is the derivative order. For our simulations we will usually be using $J=2$. The direction finding function is given by:

$$
\Gamma_{M L-\text { derivative }}(\theta)=10 \log _{10} \frac{1}{\left|\boldsymbol{w}_{\theta}^{H} \boldsymbol{a}_{f_{0}, \theta}\right|}
$$

Parameter $\chi$ is set to 1 while the bounds on $\beta$ are given by the following inequalities:

$$
\beta=\eta \frac{\operatorname{mean}\left\{\boldsymbol{a}^{H}\left(f_{0}, \theta\right) \boldsymbol{R}_{a v}^{-1} \boldsymbol{a}\left(f_{0}, \theta\right)\right\}}{\gamma_{\max }, \boldsymbol{R}_{a v}^{-1}},
$$

where, $0<\eta<1$. $\gamma_{\max }, \boldsymbol{R}_{a v}^{-1}$ is the maximum eigenvalue of the matrix $\boldsymbol{R}_{a v}^{-1}$.

\subsubsection{Frequency Focusing Model (Robust Auto-Focusing Scheme) $[36]$}

In this model the main goal is to calculate a covariance matrix that can be used with narrowband DOA techniques. This is achieved through the use of appropriate focusing matrices that transform all the the frequency dependent steering vectors to a steering vector at a frequency, $f_{0}$, for any direction $\theta$. This is illustrated by the following equation:

$$
\boldsymbol{T}_{i} \boldsymbol{A}\left(f_{i}, \theta\right)=\boldsymbol{A}\left(f_{0}, \theta\right)
$$

Each frequency domain vector is then transformed into a new vector, given by:

$$
\boldsymbol{z}\left(f_{i}\right)=\boldsymbol{T}_{i} \boldsymbol{y}\left(f_{i}\right)
$$


The covariance matrix for $\boldsymbol{z}\left(f_{i}\right)$ assumes the form

$$
\boldsymbol{R}_{\boldsymbol{z}\left(f_{i}\right) \boldsymbol{z}\left(f_{i}\right)}=\boldsymbol{A}\left(f_{0}, \theta\right) \boldsymbol{R}_{x x}\left(f_{i}\right) \boldsymbol{A}^{H}\left(f_{0}, \theta\right)+\sigma_{n}^{2}\left(f_{i}\right) \boldsymbol{T}_{i} \boldsymbol{T}_{i}^{H}
$$

This looks very similar to the first terms of (4.9), apart from the difference that here we have $\boldsymbol{T}_{i} \boldsymbol{T}_{i}^{H}$ instead of $\boldsymbol{I}_{M}$. This transformation of vectors using focusing matrices for each frequency bin, $\boldsymbol{T}_{i}$, has the effect that the frequency dependence of the covariance matrix has been removed and all the covariance matrices of each frequency bin now shares the same subspace, which is called coherent subspace.

F. Sellone [36] has proposed an optimization problem to calculate the focusing matrix such that the matrices are unitary or they obey the condition

$$
\boldsymbol{T}_{i} \boldsymbol{T}_{i}^{H}=\boldsymbol{I}_{M}, \quad i \in[1, . ., k]
$$

The problem posed is as follows:

$$
\begin{aligned}
\boldsymbol{T}_{i}= & \arg \left\{\min _{T} \int_{-\frac{1}{2}}^{\frac{1}{2}}\left\|\boldsymbol{T} \boldsymbol{a}(f, u)-\boldsymbol{a}\left(f_{0}, u\right)\right\| d u\right\} \\
& \text { subject to } \boldsymbol{T}_{i}^{H} \boldsymbol{T}_{i}=\boldsymbol{I}_{M} .
\end{aligned}
$$

The solution to this problem can be found using Appendix B. The solution is given by:

$$
T_{i}=V_{i} U_{i}^{H}
$$

where, $V_{i}$ and $U_{i}^{H}$ are matrices found from singular value decomposition of the matrix,

$$
\boldsymbol{Q}_{i} \triangleq \int_{-\frac{1}{2}}^{\frac{1}{2}} \boldsymbol{a}(f, u) \boldsymbol{a}^{H}\left(f_{0}, u\right) d u=\boldsymbol{U}_{i} \boldsymbol{\Lambda}_{i} \boldsymbol{V}_{i}^{H} .
$$

In this formulation the sensor geometry is assumed to be a uniform linear array. For $\theta \in(0, \pi]$, the variable $u \in[-1 / 2,1 / 2]$. 


\subsubsection{Proposed Focusing Technique using MUSIC-like For- mulation}

In previous sections, we have seen two techniques focusing on calculation of covariance matrix in such a way that can be used with narrowband methods such as MUSIC, MVDR, etc. For the derivative based technique (Section 4.2.1) we don't require the knowledge of the number of sources, but for the robust auto-focusing scheme (Section 4.2.2) the knowledge of the number of sources is assumed. Another drawback of this method is that the closed form solution of the integral in (4.23) is dependent on the sensor geometry. In this Section, we will see how we can mitigate these problems.

The obvious solution is to combine the two techniques of MUSIC-like algorithm (Section 2.4.4) and focusing transformation based algorithm (Section 4.2.2). This approach would be used in the following sections to develop a compressive sensing based solution. We propose the following optimization problem to solve the problems discussed above:

$$
\begin{aligned}
\underset{\boldsymbol{w}_{\theta}}{\operatorname{minimize}} & \boldsymbol{w}_{\theta}^{H} \hat{\boldsymbol{R}}\left(f_{0}, \theta\right) \boldsymbol{w}_{\theta}, \\
\text { s.t. } & \boldsymbol{w}_{\theta}^{H}\left\{\boldsymbol{a}_{\theta} \boldsymbol{a}_{\theta}^{H}+\beta \boldsymbol{I}\right\} \boldsymbol{w}_{\theta}=c .
\end{aligned}
$$

Here, $\hat{\boldsymbol{R}}\left(f_{0}, \theta\right)$ is the focused covariance matrix calculated for each direction $\theta$. The solution, as we have seen in (Section 2.4.4), to this generalized eigenvalue problem is given by

$$
\hat{\boldsymbol{R}}\left(f_{0}, \theta\right) \boldsymbol{w}_{\theta}=\lambda_{\min }\left\{\boldsymbol{a}_{\theta} \boldsymbol{a}_{\theta}^{H}+\beta \boldsymbol{I}\right\} \boldsymbol{w}_{\theta}, \theta \in(0, \pi]
$$

For each direction, $\theta$, the matrix $\hat{\boldsymbol{R}}\left(f_{0}, \theta\right)$ is calculated by using focusing matrices for each frequency bin. The focusing matrices for each frequency bin, $i$, satisfies

$$
\boldsymbol{T}\left(f_{i}, \theta\right) \boldsymbol{a}\left(f_{i}, \theta\right)=\boldsymbol{a}\left(f_{0}, \theta\right) .
$$


Note that this focusing matrix is different from the one given in (4.17). This matrix is dependent on both frequency and direction, while the one in (4.17) is dependent only on frequency. Its consequence is that now the number of focusing matrices have increased significantly. This, however, is not a drawback as all the focusing matrices do not depend on the data measurements and can be precomputed. We propose the following optimization scheme to calculate the focusing matrices, $\boldsymbol{T}\left(f_{i}, \theta\right)$, for each direction:

$$
\begin{aligned}
\boldsymbol{T}\left(f_{i}, \theta\right)=\quad & \arg \left\{\min _{T}\left\|\boldsymbol{T} \boldsymbol{a}\left(f_{i}, \theta\right)-\boldsymbol{a}\left(f_{0}, \theta\right)\right\|\right\}, \\
& \text { subject to } \boldsymbol{T}^{H}\left(f_{i}, \theta\right) \boldsymbol{T}\left(f_{i}, \theta\right)=\boldsymbol{I}_{M} .
\end{aligned}
$$

The solution to this problem is (Appendix B) given by

$$
\boldsymbol{T}\left(f_{i}, \theta\right)=\boldsymbol{v}\left(f_{i}, \theta\right) \boldsymbol{u}\left(f_{i}, \theta\right)^{H}
$$

where, $\boldsymbol{v}\left(f_{i}, \theta\right)$ and $\boldsymbol{u}\left(f_{i}, \theta\right)^{H}$ are vectors corresponding to the largest singular value of the following matrix,

$$
\boldsymbol{Q}\left(f_{i}, \theta\right) \triangleq \boldsymbol{a}\left(f_{i}, \theta\right) \boldsymbol{a}^{H}\left(f_{0}, \theta\right)=\boldsymbol{u}\left(f_{i}, \theta\right) \lambda_{\max }\left(f_{i}, \theta\right) \boldsymbol{v}\left(f_{i}, \theta\right)^{H}
$$

The pseudo-spectrum is calculated using the following equation:

$$
\Gamma_{M L-B F}(\theta)=10 \log _{10} \frac{1}{\left|\boldsymbol{w}_{\theta}^{H} \boldsymbol{a}_{f_{0}, \theta}\right|} .
$$

For parameters given in Table 4.1, we calculated the pseudo-spectrum for the two schemes as discussed above.

Figure (4.2) shows the comparison when we use uniform linear array. Figure (4.3) shows the comparison for the circular geometry shown in Figure 4.1.

The results from both these figures show that the MUSIC-like (focusing scheme) performs better in estimating the DOA of the three sources as compared to the Robust auto-focusing scheme, both for the linear and circular geometries. 
Table 4.1: Parameters used for comparison of the two schemes (MUSIC-like focusing scheme and MUSIC Robust auto-focusing scheme )

\begin{tabular}{|l|c|}
\hline Parameter & Value \\
\hline Number of sensors & 10 \\
\hline Normalized sampling frequency $\left(f_{s}\right)$ & 1 \\
\hline Center frequency $\left(f_{c}\right)$ & 0.3 \\
\hline Bandwidth & 0.24 \\
\hline FFT points & 256 \\
\hline Frequency domain snapshots & 100 \\
\hline
\end{tabular}
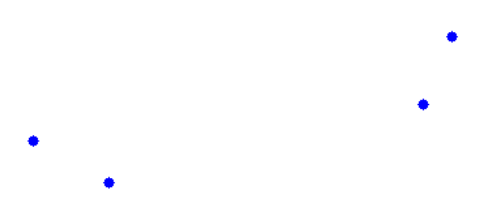

Figure 4.1: 10-element circular array used for broadband sources DOA estimation using MUSIC-like (focusing scheme) and Robust auto-focusing scheme (MUSIC). The inner circle has a radius of $3 \lambda / 2$ while the outer circle has a radius of $4 \lambda / 2$. 


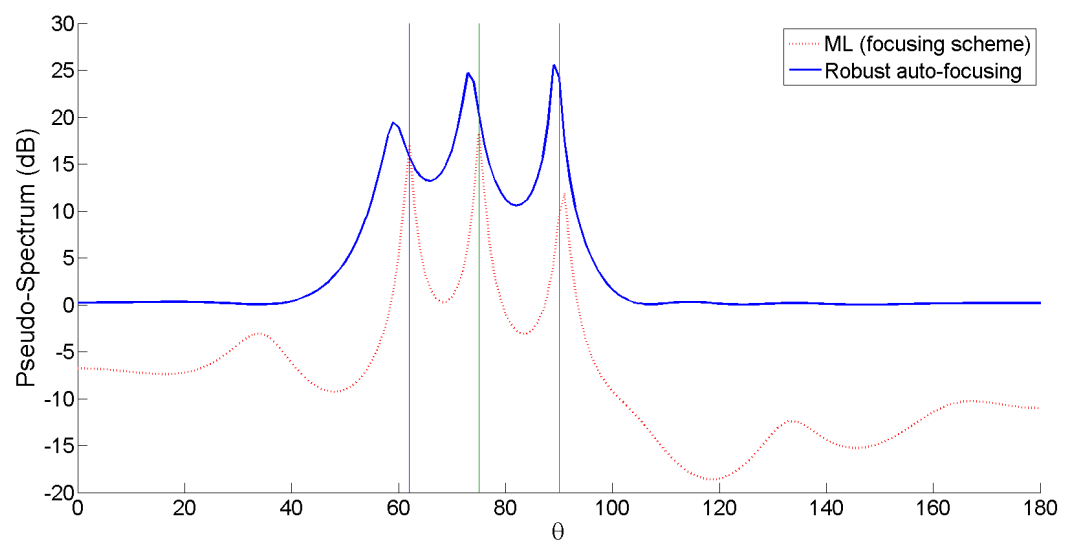

(a)

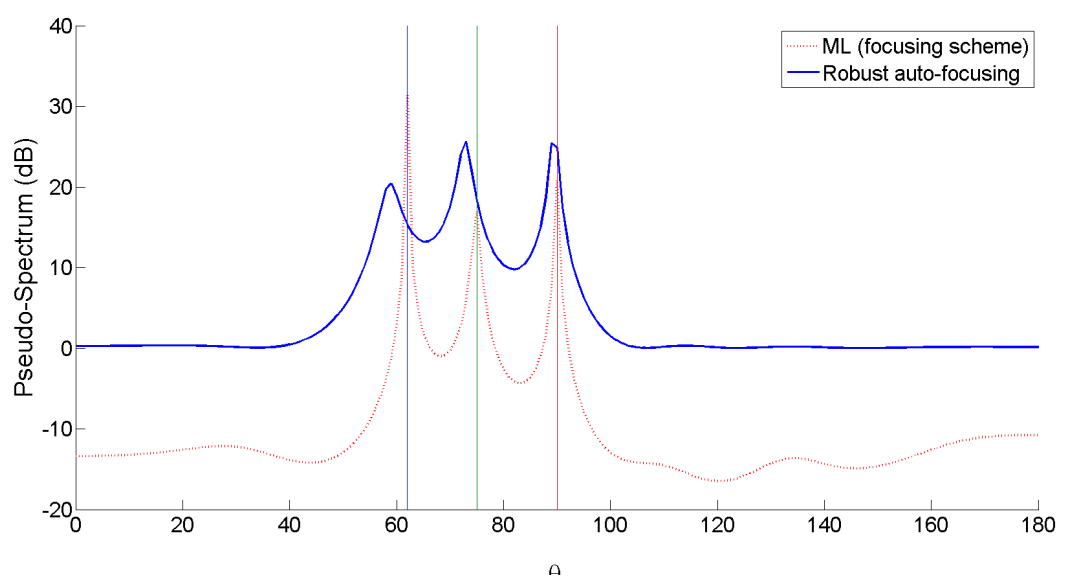

(b)

Figure 4.2: Pseudo-Spectrum comparison for uniform linear geometry for MUSIClike (focusing scheme) and Robust auto-focusing scheme (MUSIC), a) SNR value of $0 \mathrm{~dB}$ and b) SNR value of $20 \mathrm{~dB}$. 


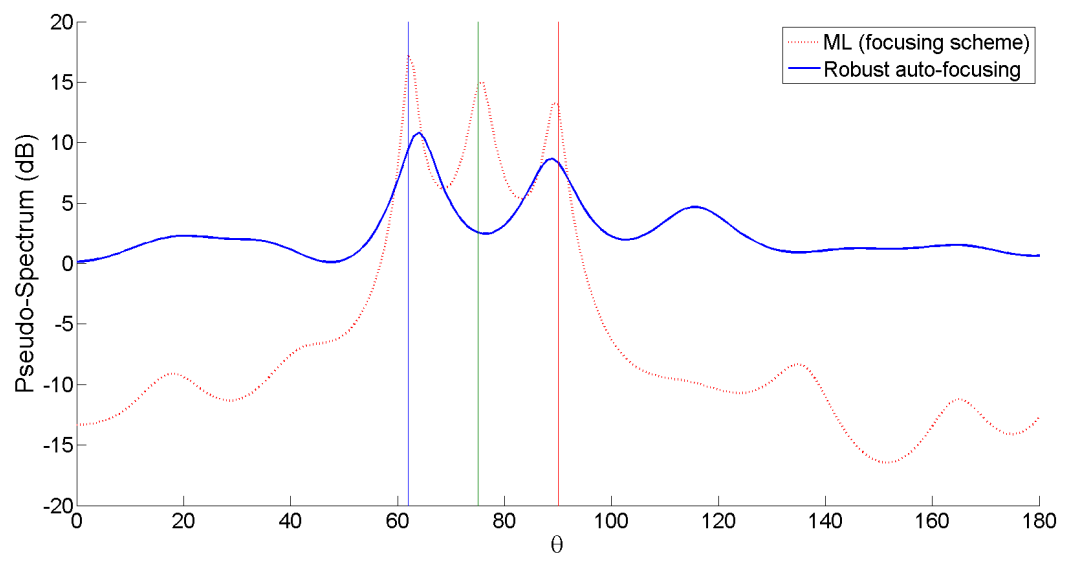

(a)

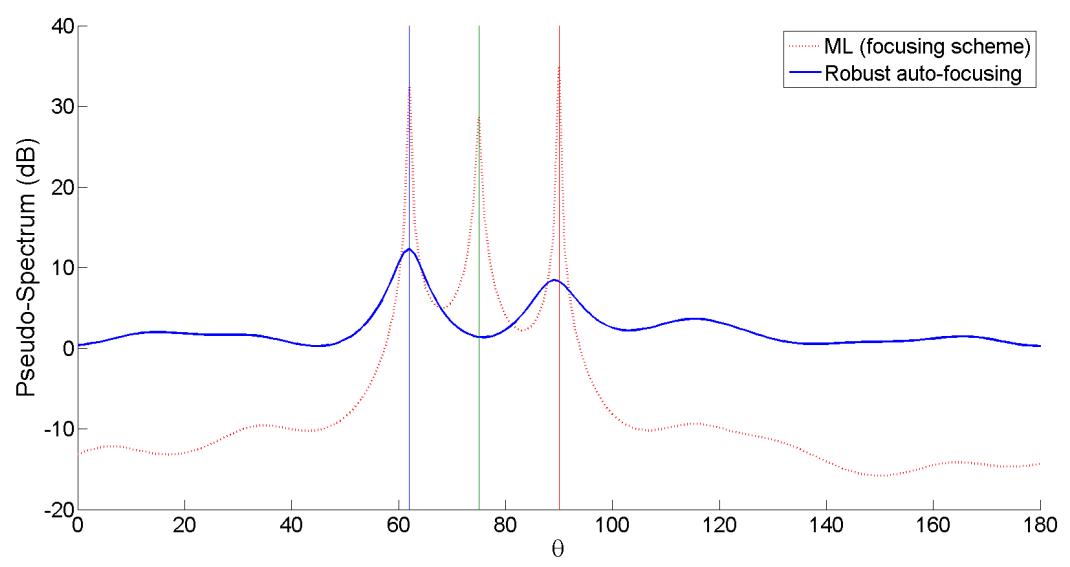

(b)

Figure 4.3: Pseudo-Spectrum comparison for circular geometry for MUSIC-like (focusing scheme) and Robust auto-focusing scheme (MUSIC) a) SNR value of 0 $\mathrm{dB}$ and b) SNR value of $20 \mathrm{~dB}$. 


\subsection{Recursive MUSIC-like Algorithms for Broad- band Sources}

In this Section, we develop several algorithms for DOA estimation of broadband sources when there are very few snapshots available. The main idea of the RML is extended to broadband model and several algorithms are developed that are capable of estimating the DOA of broadband sources in compressive scenarios.

\subsubsection{Derivative Based Recursive MUSIC-like Algorithm}

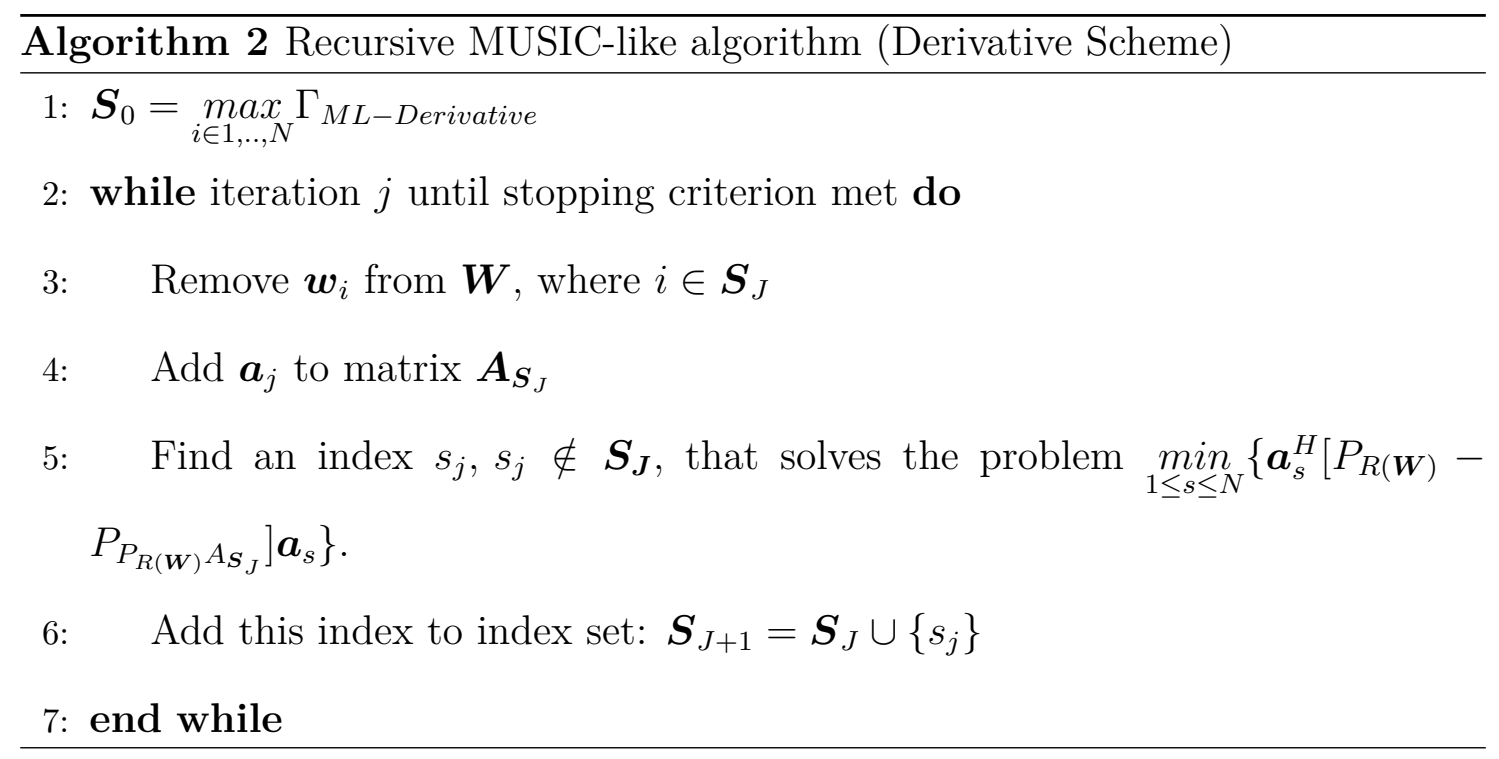

In this Section, we propose a recursive MUSIC-like algorithm based on Taylor Series Expansion formulation of the steering vectors and covariance matrix as discussed in Section 4.2.1. Although the algorithm discussed in Section 4.2.1 works for unknown number of sources, but it still has some limitations. One limitation is that this algorithm does not perform well when we have a rank deficient covari- 
ance matrix. The second limitation is that the calculation of the bounds of the parameter $\beta$ depend on the inversion of the covariance matrix.

In the case of compressive sensing, when we have a very few snapshots, the covariance matrix becomes rank deficient. The exact value of the snapshots that are required to make the covariance matrix full rank, is $r \geq M$, where $r$ is the number of snapshots. One limitation on the snapshots is that these vectors need to be linearly independent, or

$$
\operatorname{rank}(\boldsymbol{y})=M
$$

Algorithm 2 gives description of this algorithm.

\section{Stoppage Criterion}

Using similar argument as in Section 3.3.1, we use the following stoppage criterion:

1. We iterate till the maximum iterations are reached. The value of maximum iterations is set to $\max$.

2. At every iteration we find the index set $S_{J}$. If $\eta[J]<\mu$, we stop the iterations. Where

$$
\eta[J]=\frac{\left\|\left(\boldsymbol{I}-P_{\boldsymbol{A}_{\boldsymbol{S}_{J}}}\right) \boldsymbol{R}\right\|_{2}}{\left\|\boldsymbol{I}-P_{\boldsymbol{A}_{\boldsymbol{S}_{J}}}\right\|_{2}} .
$$

\section{Bounds for $\beta$}

We have already discussed in Section 3.3.2 the calculation of the bounds of the parameter $\beta$ in such a a way as to avoid the inversion of covariance matrix. The same approach is taken here to calculate the bounds. Following are the bounds for the parameter $\beta$ : 


$$
\frac{\lambda_{\boldsymbol{R}, \min }}{\xi_{\max }-\lambda_{\boldsymbol{R}, \min }}<\beta<\frac{\lambda_{\boldsymbol{R}, \min }}{\xi_{\min }-\lambda_{\boldsymbol{R}, \min }},
$$

where, $\xi_{\text {max }}=\max _{\theta \in \text { supp } \boldsymbol{X}}\left\{\boldsymbol{a}_{\theta}^{H} \boldsymbol{R} \boldsymbol{a}_{\theta}\right\}$ and $\xi_{\min }=\underset{\theta \notin \text { supp } \boldsymbol{X}}{\min }\left\{\boldsymbol{a}_{\theta}^{H} \boldsymbol{R} \boldsymbol{a}_{\theta}\right\}$.

\subsubsection{Frequency Focusing Based Recursive MUSIC-like Al-} gorithm

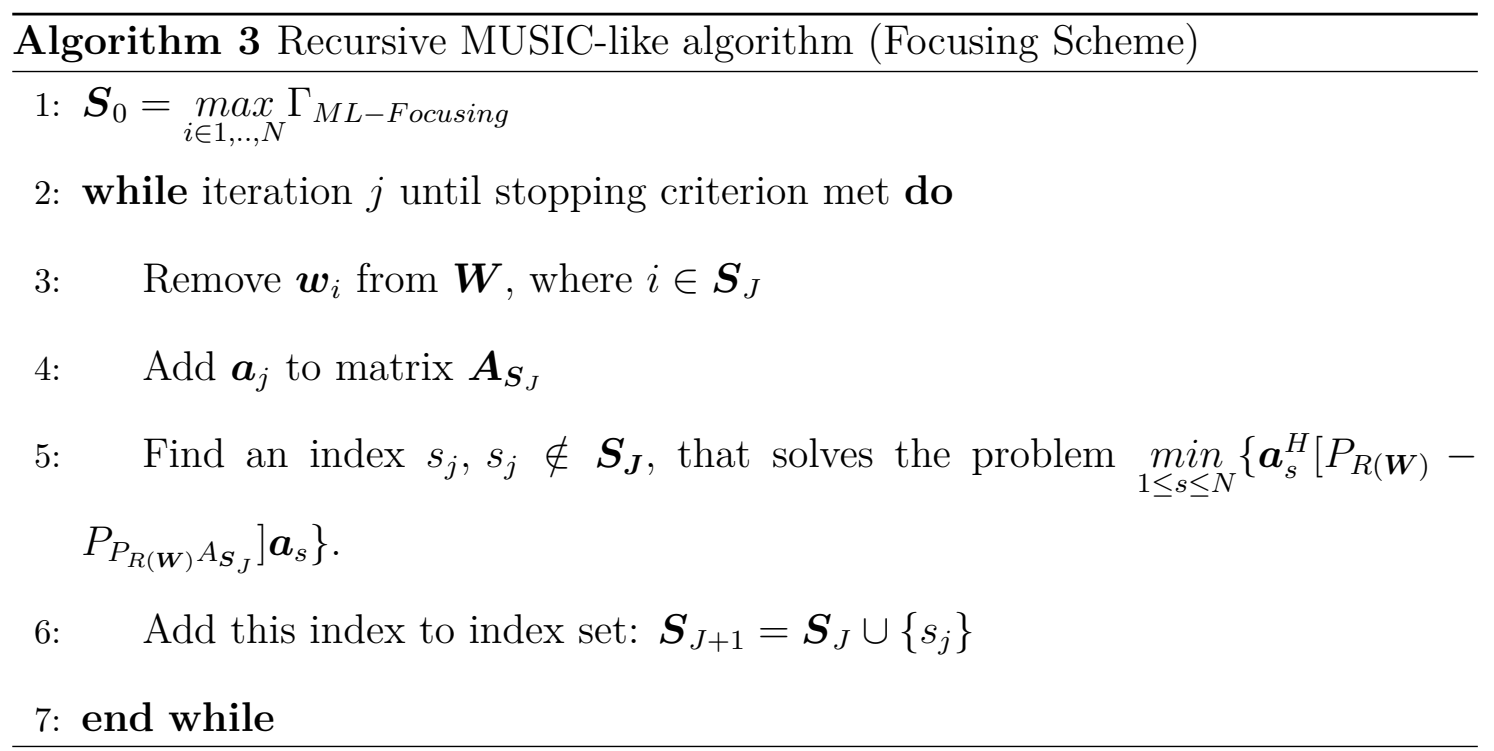

In this Section, we propose a compressive sensing based algorithm based on the algorithm discussed in Section 4.2.2. In the robust auto-focusing algorithm the first step is to calculate a set of focusing matrices for each frequency, $f_{i}$. These focusing matrices are then used to generated a focused covariance matrix.

\section{Stoppage Criterion}

Using similar argument as in Section 3.3.1, we use the following stoppage criterion: 
1. We iterate till the maximum iterations are reached. The value of maximum iterations is set to $\max$.

2. At every iteration we find the index set $S_{J}$. If $\eta[J]<\mu$, we stop the iterations. Where

$$
\eta[J]=\frac{\left\|\left(\boldsymbol{I}-P_{\boldsymbol{A}_{\boldsymbol{S}_{J}}}\right) \boldsymbol{R}\right\|_{2}}{\left\|\boldsymbol{I}-P_{\boldsymbol{A}_{\boldsymbol{S}_{J}}}\right\|_{2}} .
$$

\section{Bounds for $\beta$}

We have already discussed in Section 3.3.2 the calculation of the bounds of the parameter $\beta$ in such a a way as to avoid the inversion of covariance matrix. The same approach is taken here to calculate the bounds. Following are the bounds for the parameter $\beta$ :

$$
\frac{\lambda_{\boldsymbol{R}, \min }}{\xi_{\max }-\lambda_{\boldsymbol{R}, \min }}<\beta<\frac{\lambda_{\boldsymbol{R}, \min }}{\xi_{\min }-\lambda_{\boldsymbol{R}, \min }},
$$

where, $\xi_{\max }=\max _{\theta \in \operatorname{supp} \boldsymbol{X}}\left\{\boldsymbol{a}_{\theta}^{H} \boldsymbol{R} \boldsymbol{a}_{\theta}\right\}$ and $\xi_{\min }=\underset{\theta \notin \operatorname{mipp} \boldsymbol{X}}{\min }\left\{\boldsymbol{a}_{\theta}^{H} \boldsymbol{R} \boldsymbol{a}_{\theta}\right\}$.

\subsubsection{Bearing-Frequency Focusing Based Recursive MUSIC- like Algorithm}

In this Section, we propose Recursive MUSIC-like (RML) algorithm based on the derivation of Section 4.2.3. In this algorithm, the focusing is achieved in both the domains of frequency as well as the bearing.

We have already seen the comparison of this method with the existing robust auto-focusing technique for the non-iterative case. Further results are presented in the results Section.

Algorithm 4 gives us the detailed description of this algorithm. 


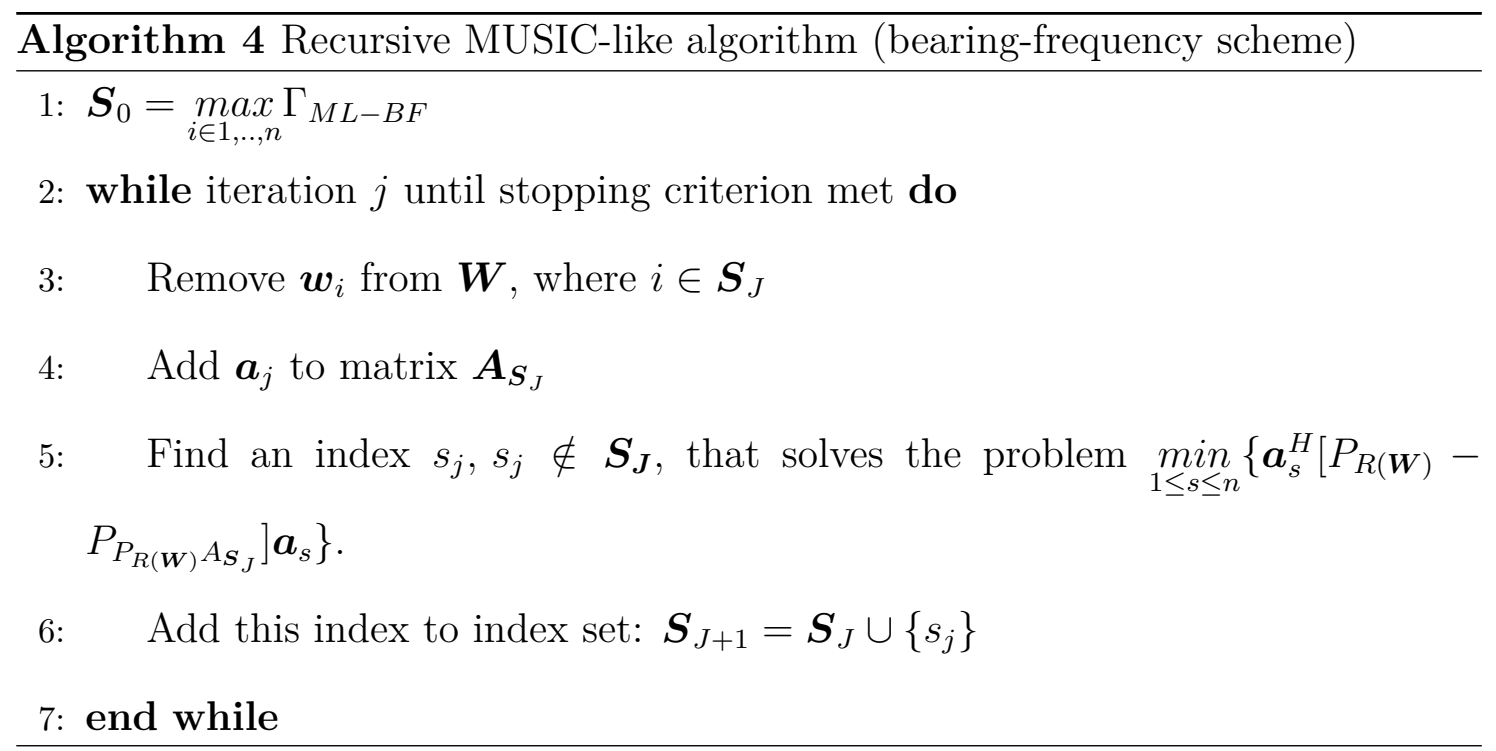

\section{Stoppage Criterion}

Using similar argument as in Section 3.3.1, we use the following stoppage criterion:

1. We iterate till the maximum iterations are reached. The value of maximum iterations is set to $\max$.

2. At every iteration we find the index set $S_{J}$. If $\eta[J]<\mu$, we stop the iterations. Where

$$
\eta[J]=\frac{\left\|\left(\boldsymbol{I}-P_{\boldsymbol{A}_{\boldsymbol{S}_{J}}}\right) \boldsymbol{R}\right\|_{2}}{\left\|\boldsymbol{I}-P_{\boldsymbol{A}_{\boldsymbol{S}_{J}}}\right\|_{2}}
$$

\section{Bounds for $\beta$}

We have already discussed in Section 3.3.2 the calculation of the bounds of the parameter $\beta$ in such a a way as to avoid the inversion of covariance matrix. The same approach is taken here to calculate the bounds. Following are the bounds for the parameter $\beta$ : 


$$
\frac{\lambda_{\boldsymbol{R}, \min }}{\xi_{\text {max }}-\lambda_{\boldsymbol{R}, \min }}<\beta<\frac{\lambda_{\boldsymbol{R}, \min }}{\xi_{\text {min }}-\lambda_{\boldsymbol{R}, \min }},
$$

where, $\xi_{\max }=\max _{\theta \in \text { supp } \boldsymbol{X}}\left\{\boldsymbol{a}_{\theta}^{H} \boldsymbol{R} \boldsymbol{a}_{\theta}\right\}$ and $\xi_{\min }=\underset{\theta \notin \text { supp } \boldsymbol{X}}{\min }\left\{\boldsymbol{a}_{\theta}^{H} \boldsymbol{R} \boldsymbol{a}_{\theta}\right\}$.

\subsection{Simultaneous Orthogonal Matching Pursuit}

\section{for Broadband Sources}

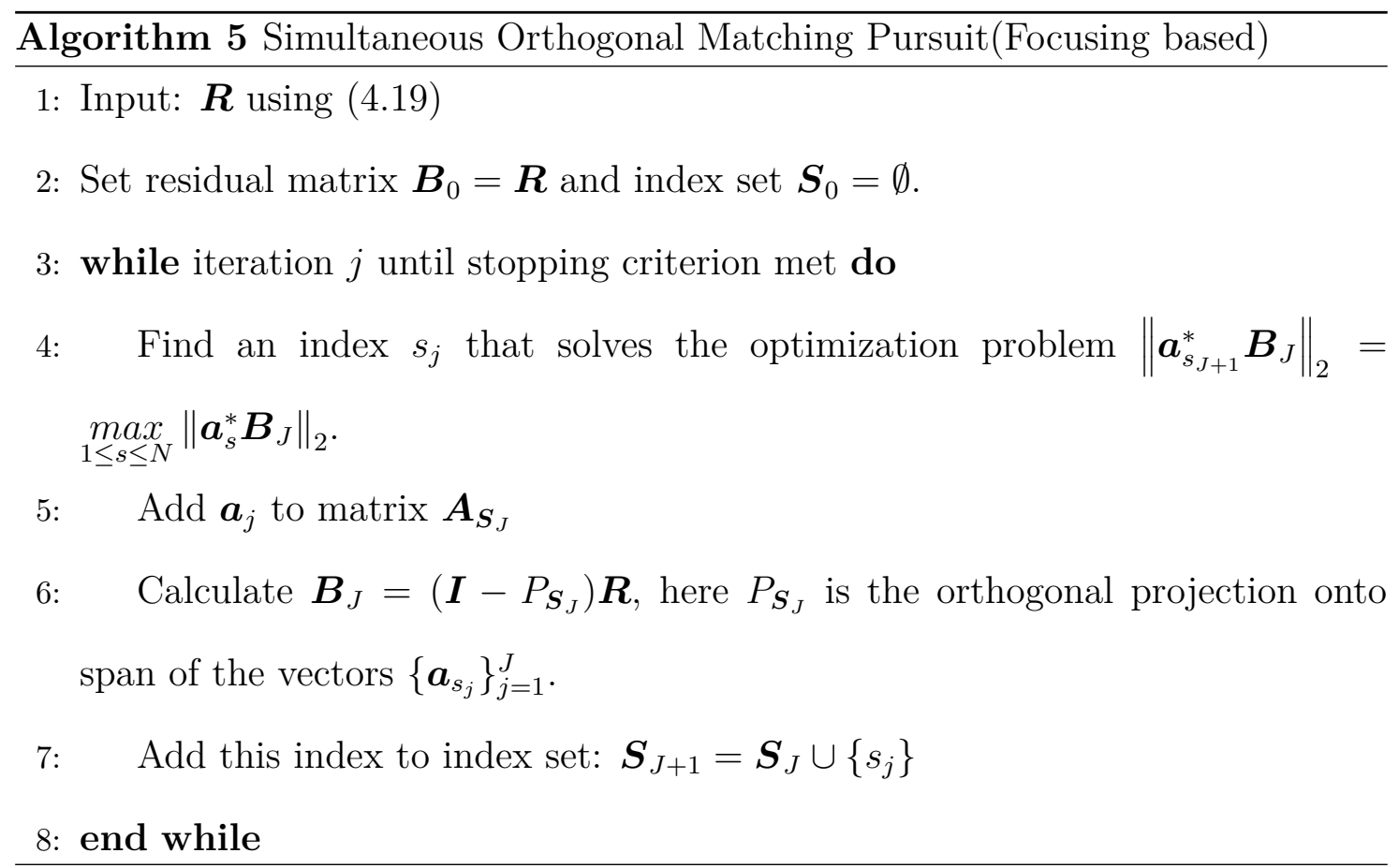

A brief description of the Simultaneous Orthogonal Matching Pursuit (SOMP) can be found in Section 2.6.1. It is a compressive sensing based algorithm. The algorithm is designed to work with multiple measurement vectors (MMV), as compared to the Orthogonal Matching Pursuit (OMP) algorithm, that uses a single 
snapshot of data. The CS bound for this algorithm is less than that of MUSIC algorithm which in contrast is $K \leq M-1$. But we would like to study this algorithm when the CS bound is satisfied and compare it with the other Recursive MUSIC-like algorithms for broadband sources.

The SOMP algorithm for narrowband sources is quite straightforward. But in case of broadband sources, the basic assumptions of this algorithm are violated, as the basis vectors are no more almost orthogonal, which affects the mutual coherence, $\mu(\boldsymbol{\Phi}, \boldsymbol{\Psi})(2.43)$

The signal model is the same as discussed earlier in this Chapter:

$$
\boldsymbol{y}(f)=\boldsymbol{A}(f, \Theta) \boldsymbol{x}(f)+\boldsymbol{n}(f)
$$

Similar to the SOMP algorithm for narrowband sources, as discussed in Section 2.6.1, all the frequency domain snapshots of $\boldsymbol{y}$ are stacked into a matrix $\boldsymbol{Y}$. This matrix can be provided as an input to the SOMP algorithm. The problem with this matrix is that it is not made up of near orthogonal basis vectors, as the steering vectors are frequency dependent, in the case of broadband signals.

We propose to use covariance matrix for each frequency bin, calculated in the following manner:

$$
\boldsymbol{R}\left(f_{i}\right)=\sum_{n=1}^{r} \boldsymbol{y}\left(f_{i}, n\right) \boldsymbol{y}^{H}\left(f_{i}, n\right)
$$

Here, $r$ represents the number of time domain snapshots.

After, covariance matrix for each bin is calculated, we use the focusing matrices (4.19) to calculate the final covariance matrix, $\boldsymbol{R}$. This covariance matrix is now fed to the the algorithm summarized as Algorithm 5.

To compare the proposed frequency focusing based SOMP algorithm with narrowband MUSIC algorithm as well as the robust auto-focusing algorithm, we per- 


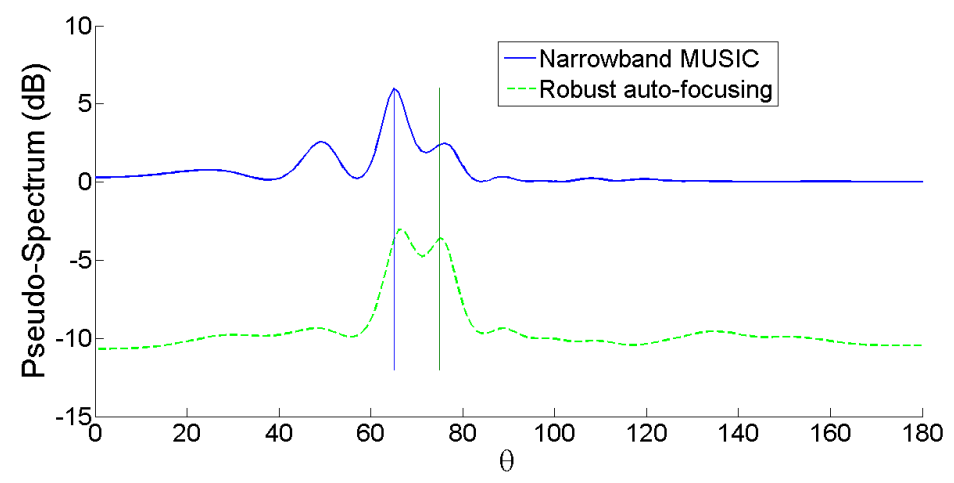

Figure 4.4: Comparison of Narrowband MUSIC algorithm with the Robust autofocusing algorithm (Section 4.2.2) for two sources located at 65 and 75 degrees respectively. SNR is $10 \mathrm{~dB}$, the number of sensors $\mathrm{M}=20$ in uniform linear array configuration. Two frequency bins have been used, while, the source signal normalized bandwidth 0.1. The vertical lines indicate the direction of the two sources. 


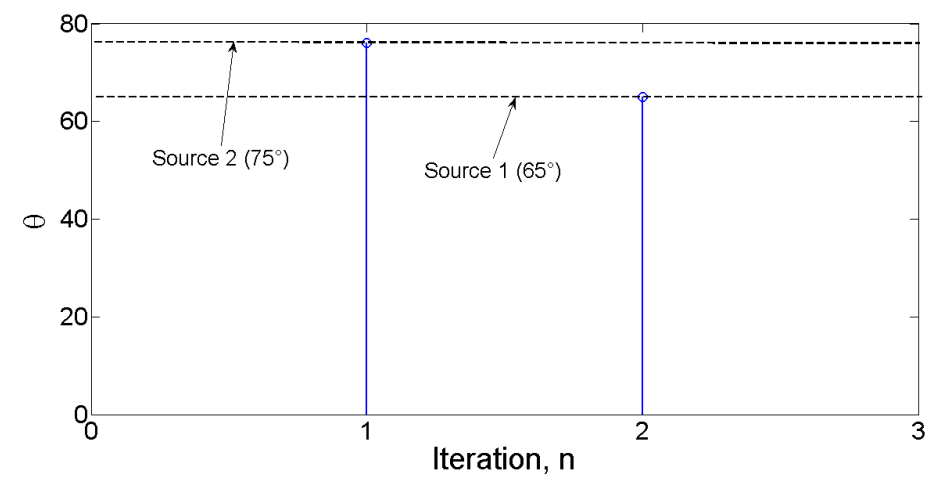

Figure 4.5: Two iterations of Frequency focusing based SOMP algorithm. The two dotted lines show the actual direction of the two sources at 65 and 75 degrees respectively. This simulation was performed for signal with 10-dB SNR, the number of sensors were $\mathrm{M}=20$ in uniform linear array configuration. Two frequency bins have been used, while, the source signal normalized bandwidth 0.1. 
formed a simulation based on the following parameters. SNR of the signal was set to $10 \mathrm{~dB}$. We used 20 sensors in uniform linear array with minimum separation of $\lambda / 2$. There were two sources at 65 degrees and 75 degrees respectively. The sources were broadband and generated using frequency model for broadband sources (4.1) with two frequency bins and normalized bandwidth of 0.1 . The phases and amplitudes of the signals were randomly generated. Figure 4.4 shows the comparison of the narrowband MUSIC and robust auto-focusing algorithm (Section 4.2.2). The results of the simulation indicate that the narrowband MUSIC algorithm failed to estimate one source. While, the robust auto-focusing scheme does have two high peaks but the estimates are not exact. Figure 4.5 shows the results of two iterations of the frequency focusing SOMP algorithm for the same parameters as discussed. It successfully located both the sources without any error.

We have used the same stoppage criterion for frequency focusing SOMP as used for the algorithms developed earlier in this chapter. The stoppage crtierion is summarized below:

1. We iterate till the maximum iterations are reached. The value of maximum iterations is set to $\max$.

2. At every iteration we find the index set $S_{J}$. If $\eta[J]<\mu$, we stop the iterations. Where

$$
\eta[J]=\frac{\left\|\left(\boldsymbol{I}-P_{\boldsymbol{A}_{\boldsymbol{S}_{J}}}\right) \boldsymbol{R}\right\|_{2}}{\left\|\boldsymbol{I}-P_{\boldsymbol{A}_{\boldsymbol{S}_{J}}}\right\|_{2}} .
$$

\subsection{Simulation Results}

In this Section, we evaluate the performance of the four algorithms discussed in Sections 4.3.1, 4.3.2, 4.3.3 and 4.4. All of these algorithms are recursive, developed 
for compressive scenarios, but are applicable in non-compressive settings (when we have a large number of snapshots) as well. In a sense, these algorithms have a wider application as compared to MUSIC based algorithms like CSSM. Here, our aim is to evaluate the performance of these algorithms for both the compressive and noncompressive situations. In non-compressive setting the algorithms are compared with a well known algorithm, CSSM, discussed before in Section 2.5.2, while in compressive scenario we compare the performance of these algorithms with each other. We study phase transition, probability of resolution and mean squared error performance of these algorithms.

\subsubsection{Simulation Setup}

For all the simulations we have used Uniform Linear Array (ULA). The distance, $d$, between adjacent sensors is set to $\lambda_{\min } / 2$, where $\lambda_{\min }$ is the wavelength corresponding to the highest frequency in the source signal given by

$$
f_{\max }=f_{c}+B W / 2 \text {. }
$$

Here, $f_{c}$, is the center frequency and $B W$ is the double sided bandwidth of the source signal. The complete set of simulation parameters are given in table 4.2. 
Table 4.2: Parameters used for comparison of the broadband RML algorithms

\begin{tabular}{|l|c|}
\hline Parameter & Value \\
\hline Number of sensors & 10 \\
\hline Normalized sampling frequency $\left(f_{s}\right)$ & 1 \\
\hline Center frequency $\left(f_{c}\right)$ & 0.3 \\
\hline Bandwidth & 0.24 \\
\hline
\end{tabular}

We have used a large bandwidth of 0.24 , which amounts to $80 \%$ of the center frequency. For montecarlo simulations we have generated the broadband source signal using the following model:

$$
\boldsymbol{x}(t)=\sum_{f=f_{\min }}^{f_{\max }} a(f, t) e^{j(2 \pi f+\phi(f, t)} .
$$

Here, $f_{\min }=f_{c}-B W / 2, a(f, t)$ is the signal amplitude which is generated randomly for each frequency bin and each time snapshot. Similarly the phase $\phi(f, t)$ is also generated randomly for each montecarlo simulation.

\subsubsection{Performance Evaluation}

In this Section, we discuss the performance evaluation strategy for the algorithms developed in this chapter. We evaluate the performance of the four algorithms in a compressive set up when the number of time-domain snapshots are very few $(r=2$ and $r=16)$ while number of frequency bins are also varied $(\mathrm{D}=16, \mathrm{D}=32$, $\mathrm{D}=128$ and $\mathrm{D}=256$ ). Using the phase transition plots we study the detection capability of different algorithms discussed in this chapter. The number of sensors, 

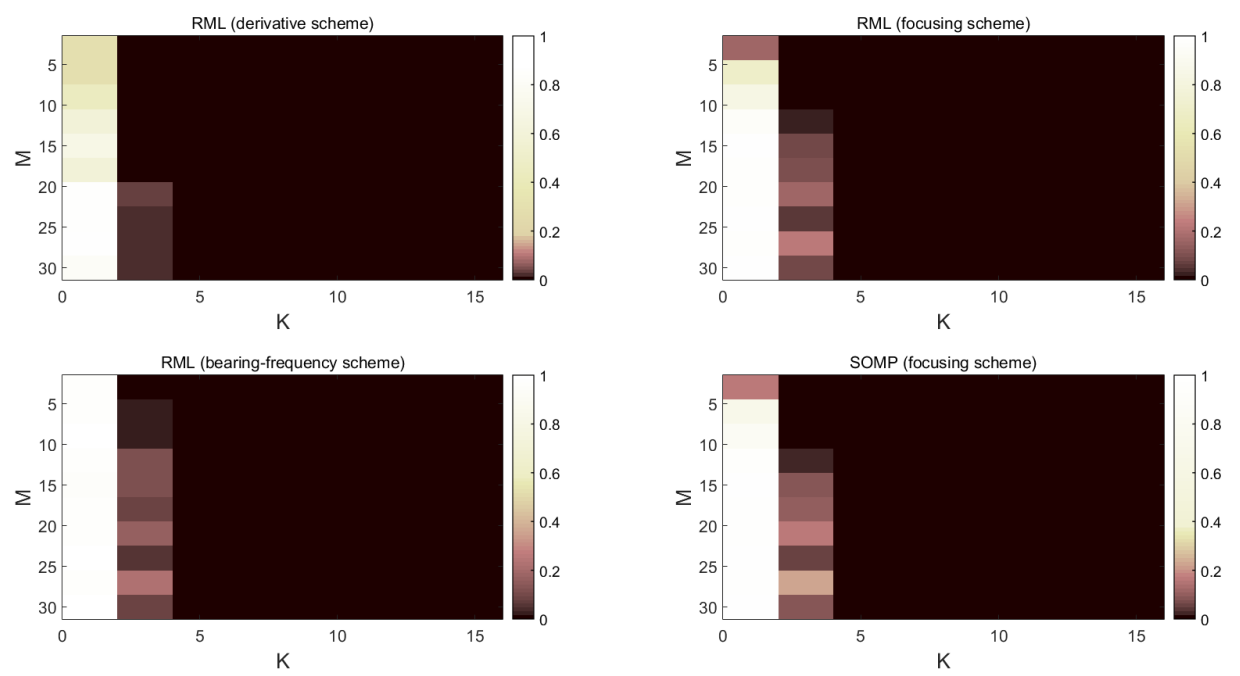

Figure 4.6: Phase transition plots for the four algorithms for $\mathrm{r}=2, \mathrm{D}=16$ and $\mathrm{SNR}=40 \mathrm{~dB}$.

$M$, is varied. Where, $M \in\{3,6,9,12,15,18,21,24,27,30\}$. The number of sources, $K$, is also varied and $K \in\{1,3,5,7,9,11,13,15\}$. The direction of the sources is chosen randomly from a uniform distribution with a domain of $N \in\{1, . ., 180\}$. The minimum separation between two sources is kept at 1 degree. All the simulations in this section were performed for 200 montecarlo iterations.

The phase transition plots, when we have 2 time domain snapshots $(r=2)$, are shown in Figures 4.6 - 4.9. Each plot has a different value for frequency domain snapshots. The color is shown as white for high detection probability $\left(P_{D}=1\right)$ while black if there was no detection $\left(P_{D}=0\right)$. The performance of other algorithms is quite similar while that of the derivative scheme is poorer as compared to the other schemes. With the increase of frequency bins, there is no visible improvement in the performance of any of the algorithms studied. SNR for these simulations was 

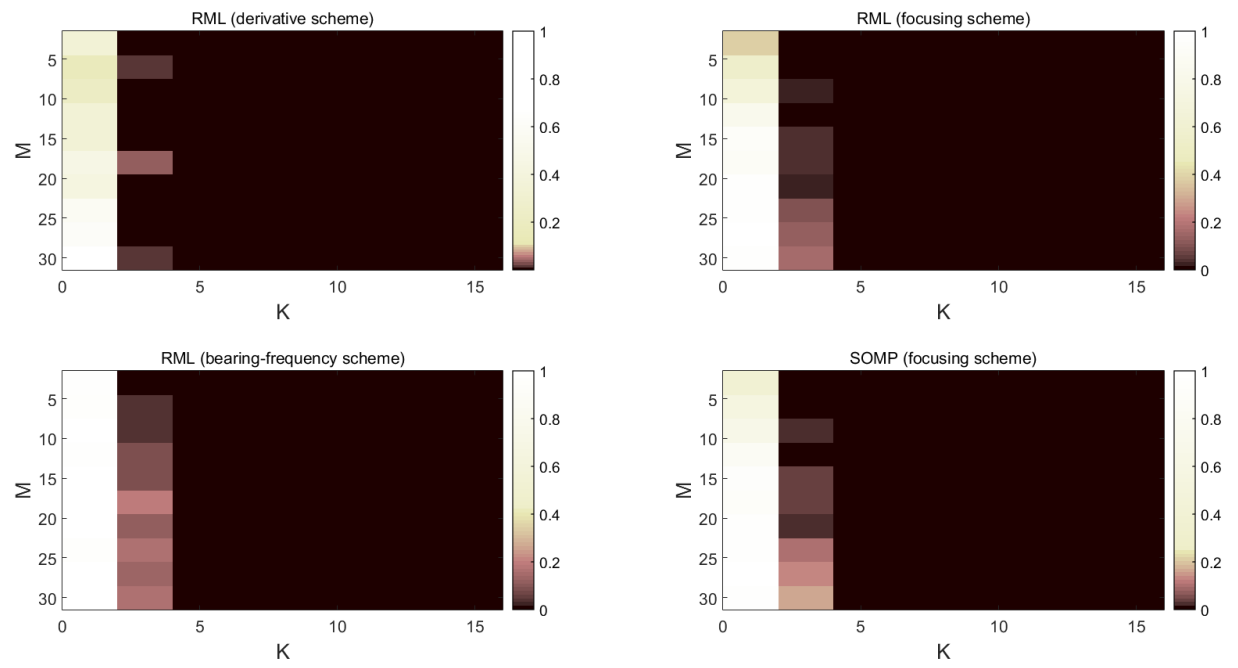

Figure 4.7: Phase transition plots for the four algorithms for $r=2, D=32$ and $\mathrm{SNR}=40 \mathrm{~dB}$
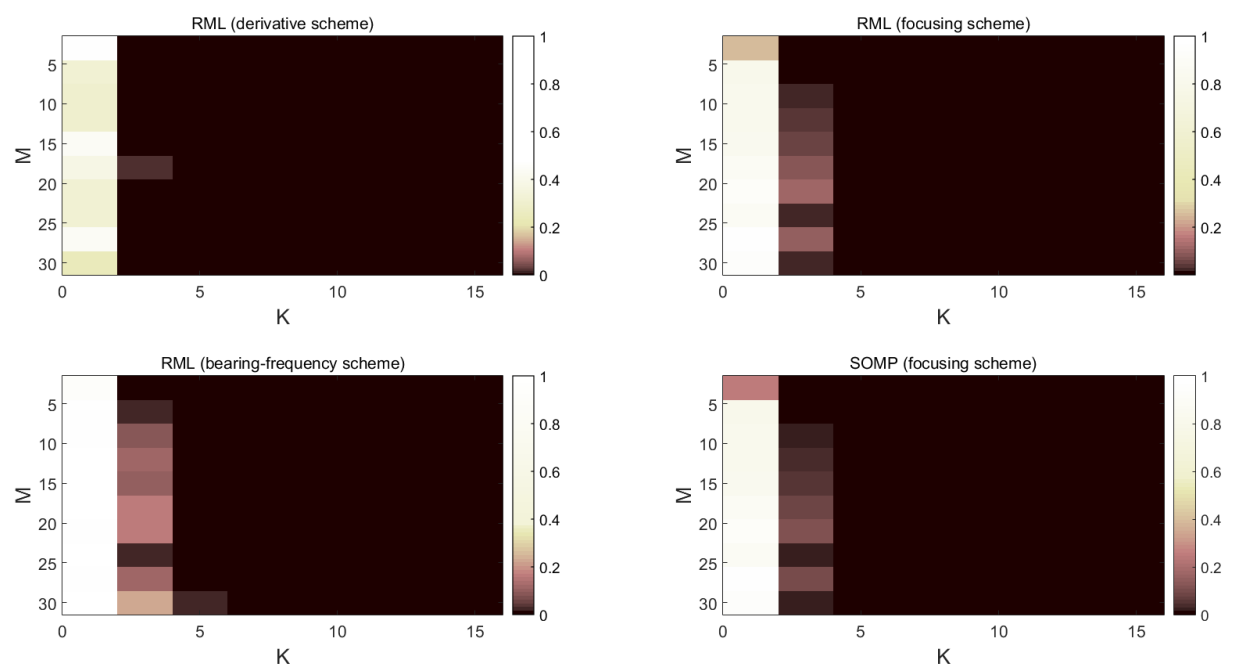

Figure 4.8: Phase transition plots for the four algorithms for $\mathrm{r}=2, \mathrm{D}=128$ and $\mathrm{SNR}=40 \mathrm{~dB}$. 

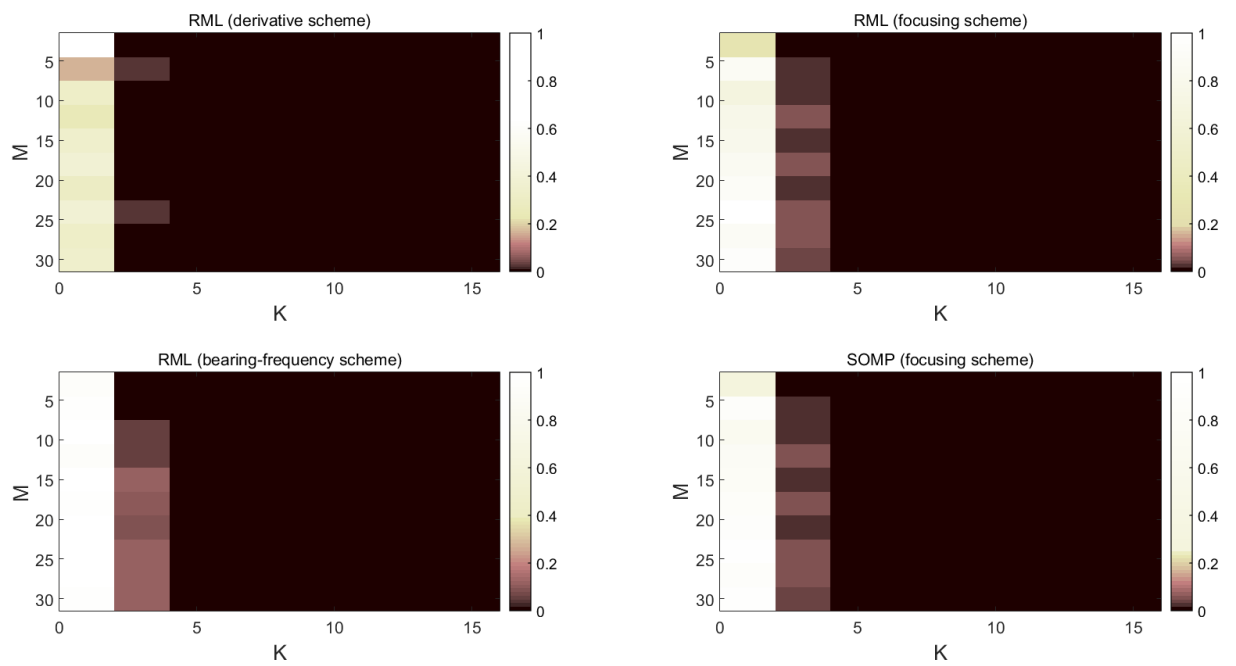

Figure 4.9: Phase transition plots for the four algorithms for $\mathrm{r}=2, \mathrm{D}=256$ and $\mathrm{SNR}=40 \mathrm{~dB}$.

kept at $40 \mathrm{db}$.

The phase transition plots, when we have 16 time domain snapshots $(r=16)$, are shown in Figures 4.10 - 4.13. Each plot has a different value for frequency domain snapshots. The color is shown as white for high detection probability $\left(P_{D}=1\right)$ while black if there was no detection $\left(P_{D}=0\right)$. The performance of the bearingfrequency is better than other algorithms, while that of the derivative scheme is poorer as compared to the other schemes. With the increase of frequency bins, there is no visible improvement in the performance of any of the algorithms studied. But comparing these results to the previous ones we can clearly see that the increase in the number of time domain snapshots does increase the detection capability of these algorithms.

For the Probability of Resolution $P_{R}$ evaluation, we define a window around 

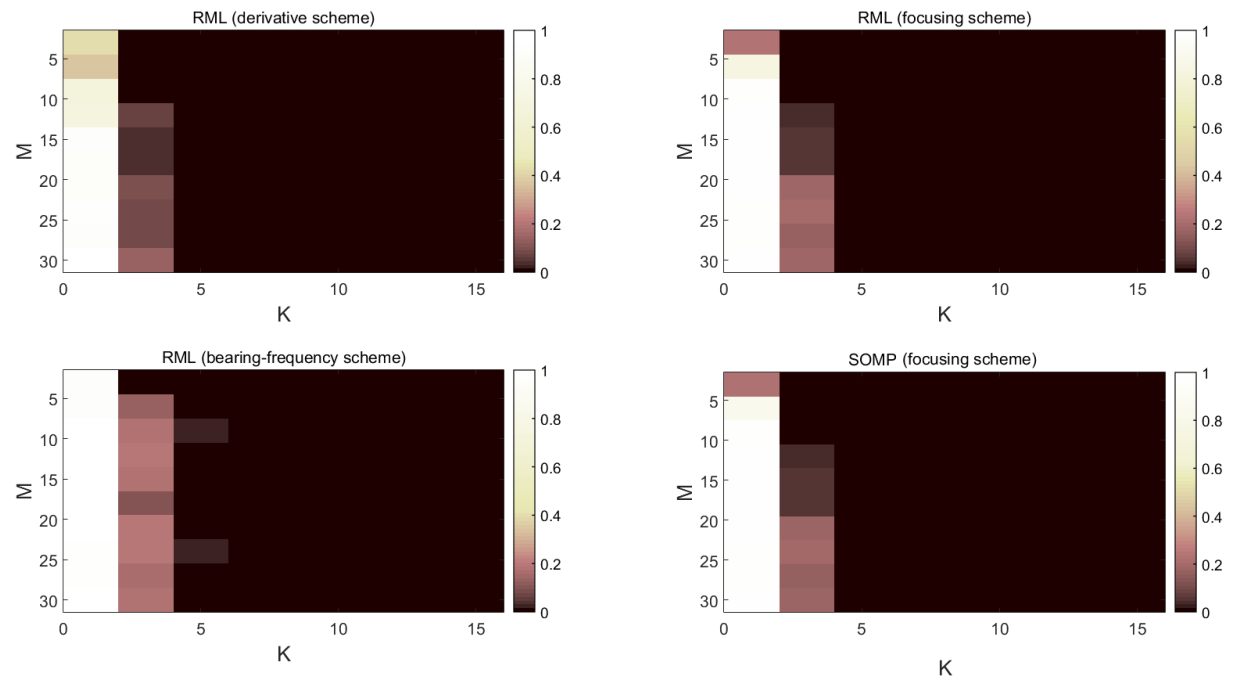

Figure 4.10: Phase transition plots for the four algorithms for $\mathrm{r}=16, \mathrm{D}=16$ and $\mathrm{SNR}=40 \mathrm{~dB}$
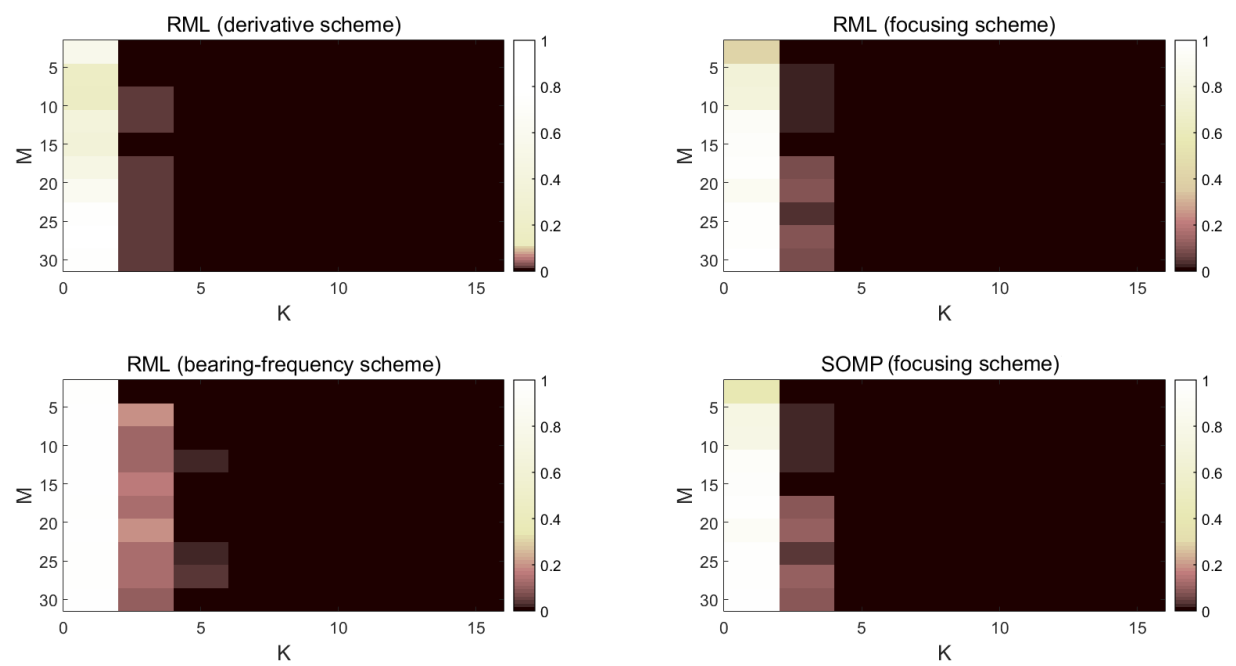

Figure 4.11: Phase transition plots for the four algorithms for $\mathrm{r}=16, \mathrm{D}=32$ and $\mathrm{SNR}=40 \mathrm{~dB}$ 

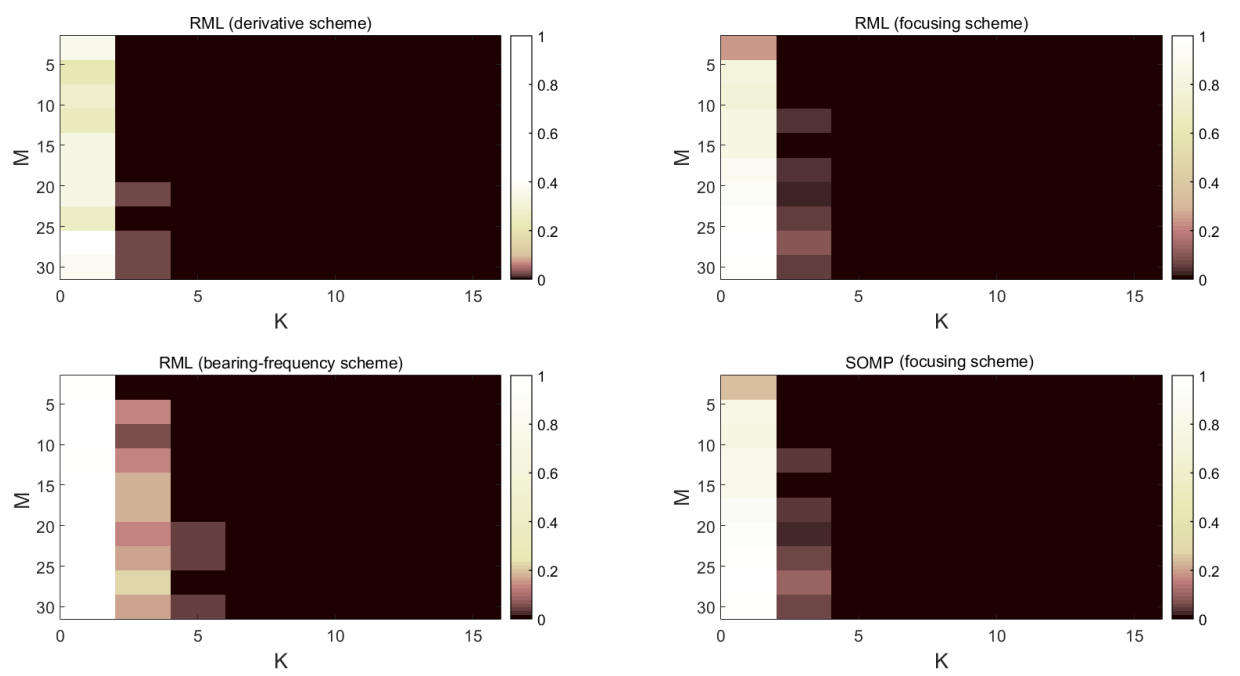

Figure 4.12: Phase transition plots for the four algorithms for $\mathrm{r}=16, \mathrm{D}=128$ and $\mathrm{SNR}=40 \mathrm{~dB}$
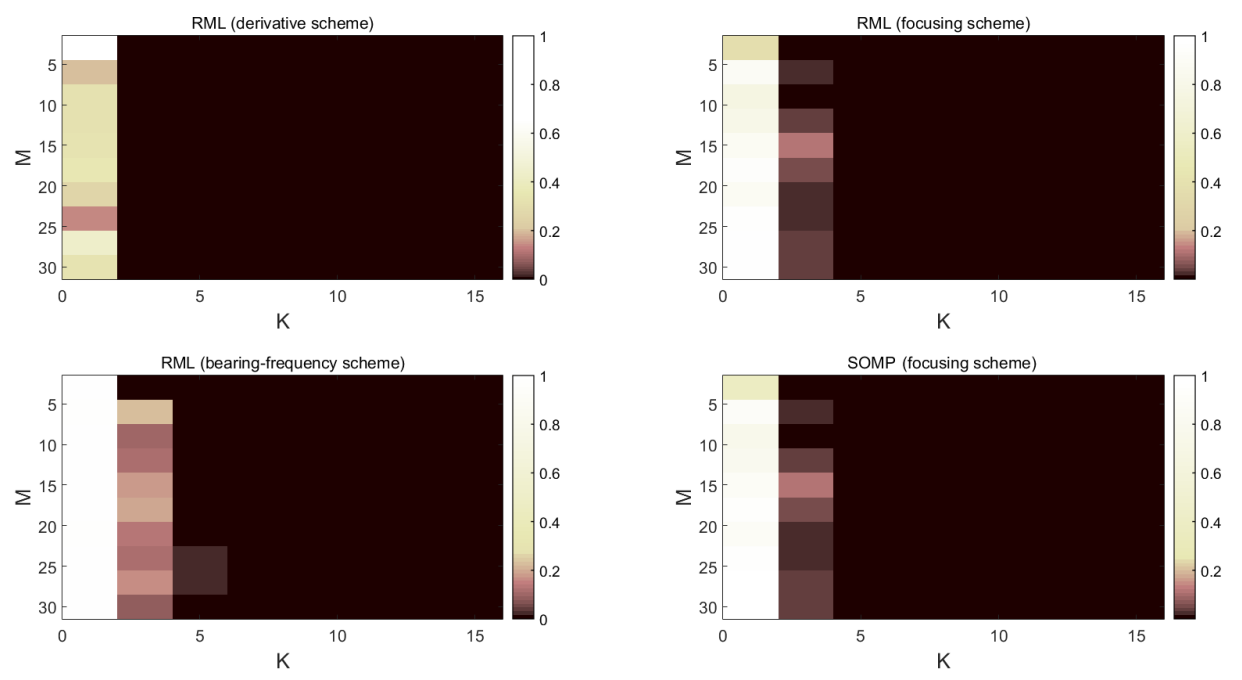

Figure 4.13: Phase transition plots for the four algorithms for $\mathrm{r}=16, \mathrm{D}=256$ and $\mathrm{SNR}=40 \mathrm{~dB}$ 


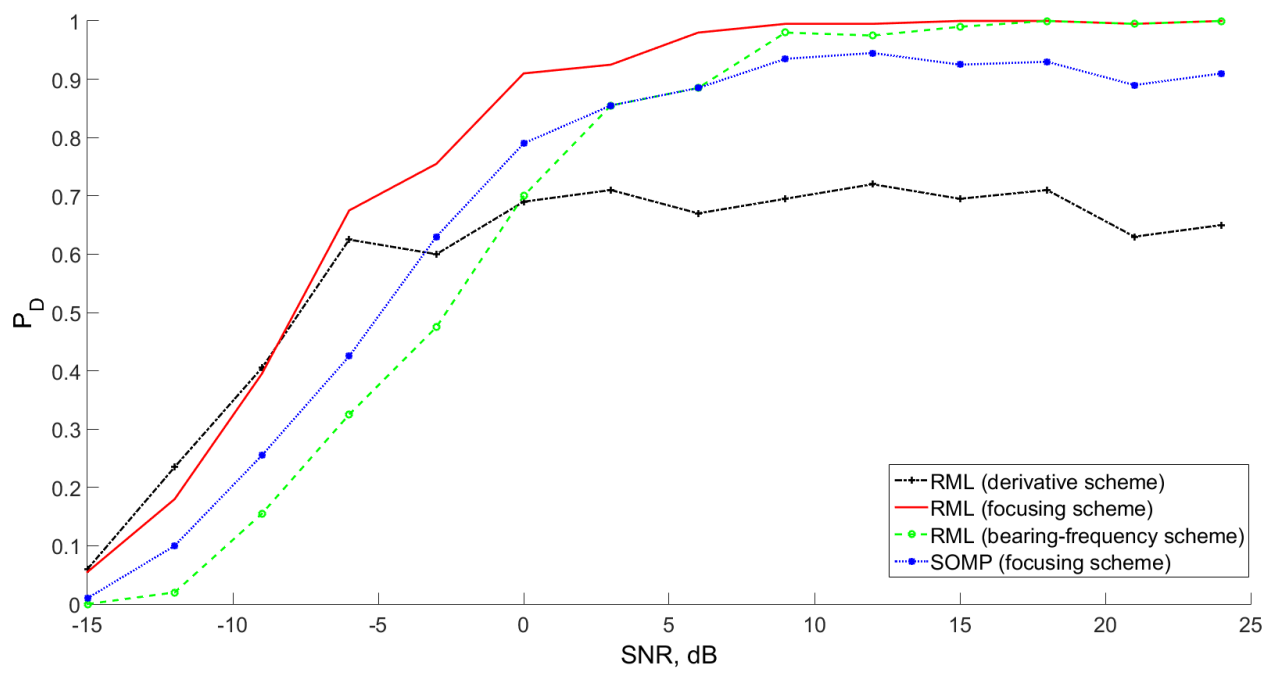

Figure 4.14: $P_{R}$ vs SNR plots for $\mathrm{r}=2$ and $\mathrm{D}=32$ and $\mathrm{M}=31$.

the actual angle of arrival, $\theta$, if the estimated angle $\hat{\theta}$ lies within that window, it is considered detected. For more that one target (as in our case, we have three targets), for each iteration of the Montecarlo simulation, if all the target-peaks were within this window, we considered the detection successful, if any of the estimated DOA lied outside the window, target resolution is considered a failure.

For three sources at 78, 94 and 100 degrees azimuth, we ran the four algorithm for 200 Montecarlo simulations. The average of these simulations for compressive scenario, when we have very few time domain snapshots $(\mathrm{r}=2)$, is shown in 4.14. The performance of frequency focusing algorithm is better than the rest of the algorithms. Derivate algorithm, is incapable of reaching the $P_{D}=1$ line even when the SNR gets very good. For non-compressive scenario, when we have ample time domain snapshots $(\mathrm{r}=100)$, we can see the performance of SOMP algorithm is notably better than the rest of the algorithms. It out-performs the CSSM algorithm 


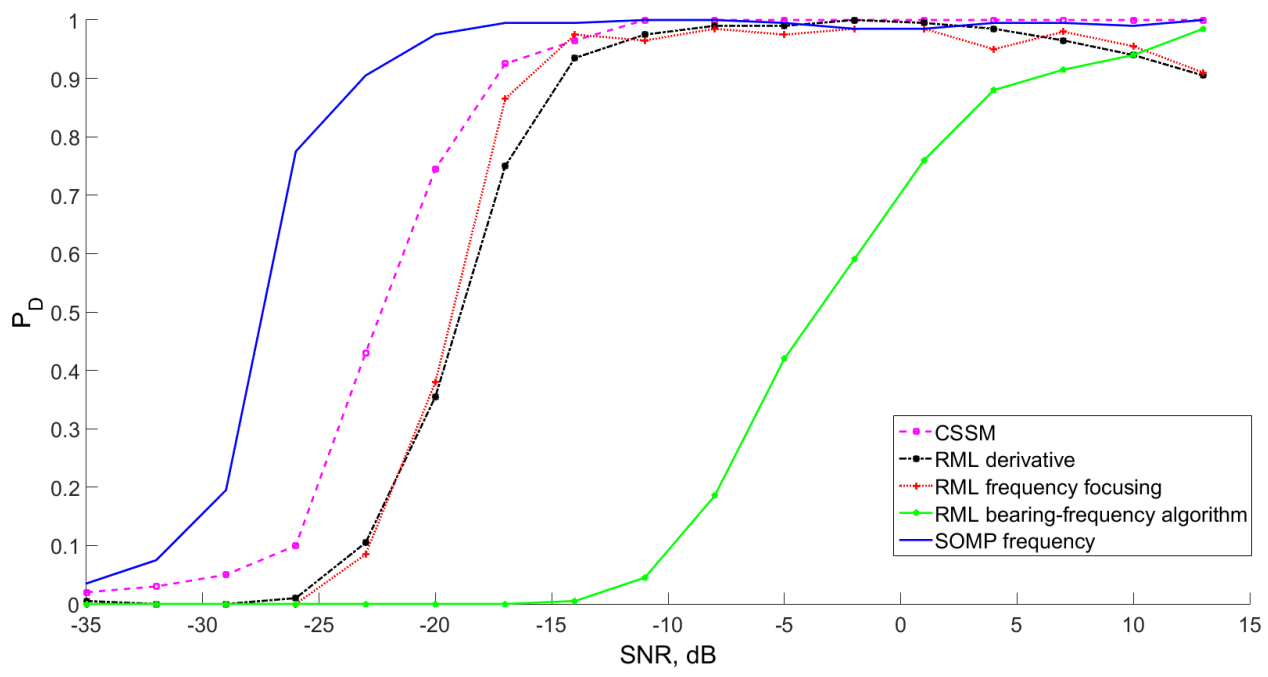

Figure 4.15: $P_{R}$ vs SNR plots for $\mathrm{r}=100$ and $\mathrm{D}=256$ and $\mathrm{M}=10$. Please note that the horizontal axis is shifted left as compared to Figure 4.14 as there are more snapshots and thus the performance is better. 
as well as the other compressive algorithms.

Next, we study the MSE of different targets in compressive and and noncompressive scenarios.

The mean squared error for each target is evaluated over $\mathrm{N}$ simulations using the following equation:

$$
M S E=\frac{1}{N} \sum_{i=1}^{N}(\theta-\hat{\theta})^{2} .
$$

The mean squared error plots for the three targets at 78, 94 and 110 degrees azimuth for the compressive case, when we have very few time domain snapshots $(r=2)$ and $D=32$ frequency bins, is shown in Fig 4.16. For sources 1 and 3, the bearing-frequency performs better than all other scheme, while its performance for target 2 is slightly poor as compared to the other algorithms. For the non compressive case, when we have large number of snapshots $(r=100)$, the bearingfrequency completely fails for SNR below -10 db. CSSM and SOMP algorithm have consistent performance for all the three targets.

\subsection{Conclusions}

Four algorithms for broad-band DOA estimation were developed and analyzed in this chapter using four approaches: derivative based, frequency focusing based, SOMP using the RML criterion developed in Chapter 3. These algorithms were analyzed and we found that the bearing-frequency is suitable for compressive scenario when SNR is high, while the SOMP scheme had consistent performance but the derivative scheme failed to perform well. 


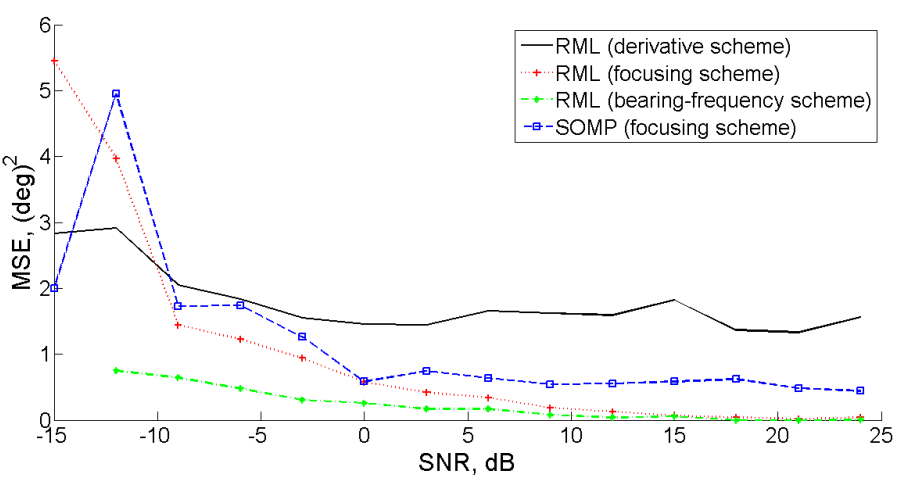

(a)

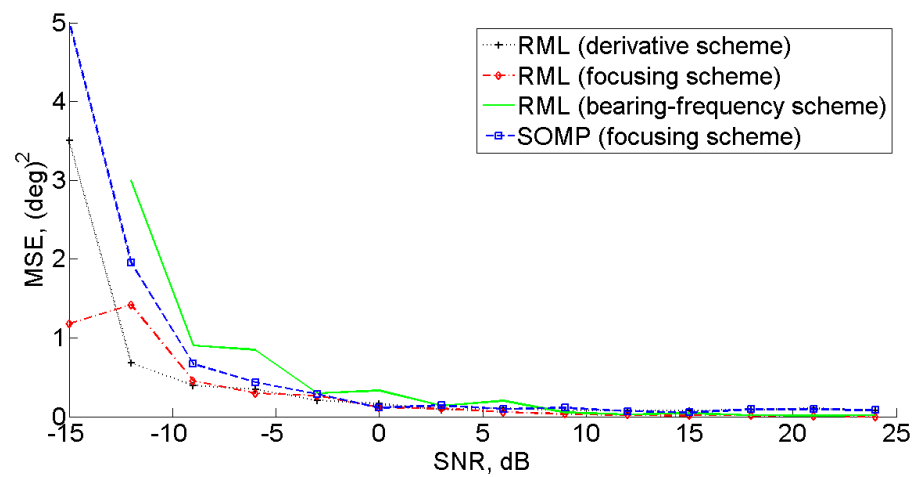

(b)

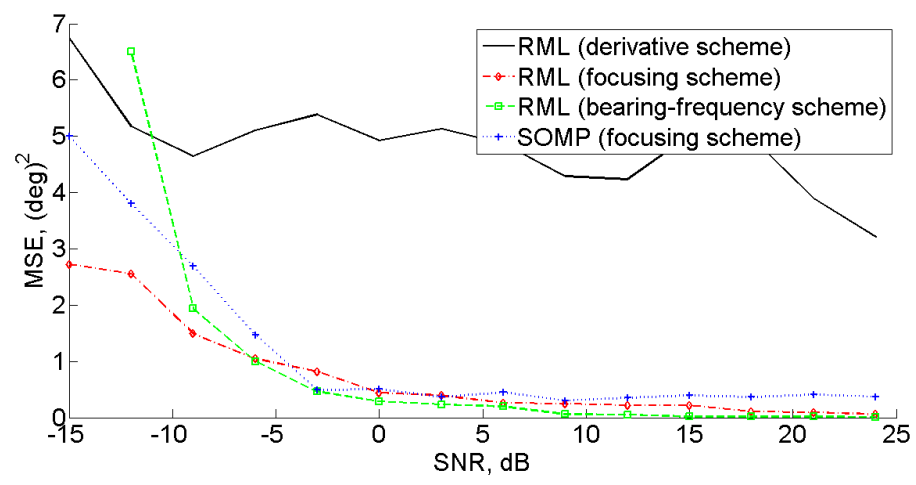

(c)

Figure 4.16: MSE plot for $\mathrm{r}=2$ and $\mathrm{D}=32$ and $\mathrm{M}=31$ a) Source 1 at 78 degrees, b) Source 2 at 94 degrees, c) Source 3 at 110 degrees. Note that the vertical axis is not constant for the three sub-figures. 


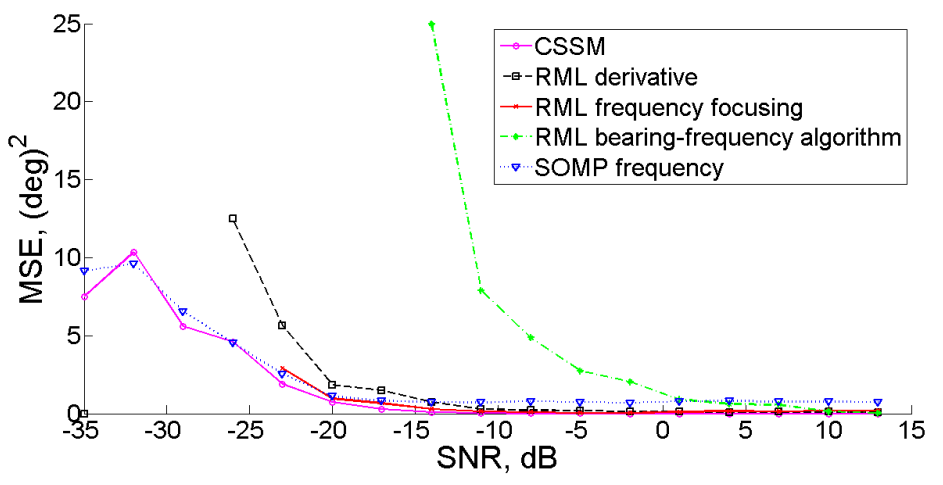

(a)

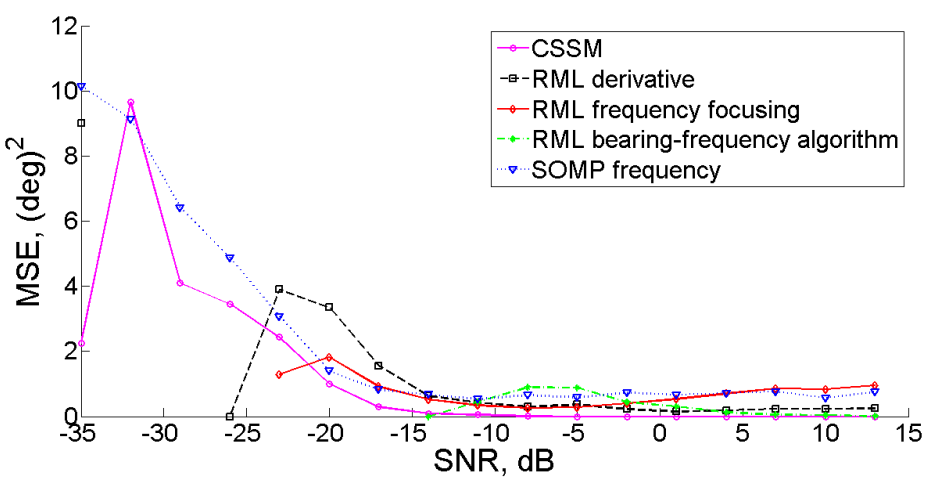

(b)

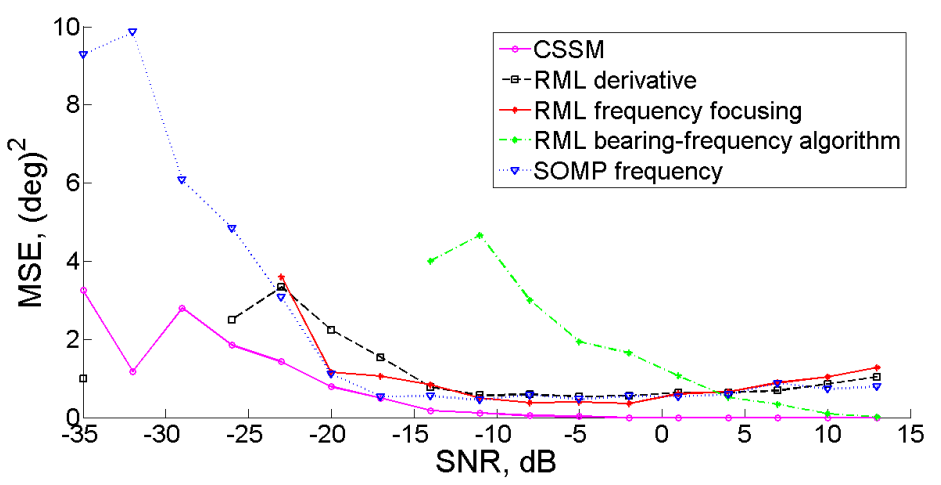

(c)

Figure 4.17: MSE plot for $\mathrm{r}=100$ and $\mathrm{D}=256$ and $\mathrm{M}=10$ a) Source 1 at 78 degrees, b) Source 2 at 94 degrees, c) Source 3 at 110 degrees. Since the number of snapshots are much larger for this figure as compared to Figure 4.16, the horizontal axis is shifted as the detection is better even at poor SNR. Also note the vertical scale is not constant for the three sub-figures. 


\section{Chapter 5}

\section{Array Geometry Design for}

\section{Narrow Band Compressive}

\section{Sensing DOA Estimation}

\section{$5.1 \quad$ Introduction}

Compressive sensing has gained considerable attention in literature recently. It has been applied to many problems including image processing, image compression, radar signal processing, etc. The problem of array geometry pertaining to CS theory has not been studied very well.

The array geometry that is commonly used is Uniform Linear Array or its 2-D counterpart, Rectangular Grid Array. The neighboring elements in these arrays, on each axis, are separated by a fixed distance, usually $\lambda / 2$. The configuration works well if we are looking for compression in frequency domain. But if the design 
objective is to compress the number of array elements, then this configuration is not suitable. A random sampling of aperture is much more suitable [20].

Herman et. al. [21] and Ender [22] studied application of compressive sensing to the radar problem and gave some initial results regarding the potential of using CS to address DOA estimation problem. Ender, in his paper [22] addresses several radar problems, including pulse compression, inverse synthetic aperture radar (ISAR) and radar direction of arrival (DOA) estimation. Both of these papers however use a geometry that is achieved through random sampling of aperture. Random sampling of aperture generates a measurement matrix that is well suited to use with CS algorithms but it is not practically feasible. There is no underlying geometry in a random sampled aperture. Usually in a randomly sampled aperture some of the elements would lie too close to each other which is not practically realizable.

The study of sparse array geometry is a rapidly developing topic. In the formation of covariance matrix for MUSIC algorithm the difference co-array is utilized instead of the usual output covariance matrix. Ma et al [41] developed a subspace augmentation technique based on Khatri-Rao product that effectively created "virtual sensors", so that the DOA for more sources can be estimated than the number of sensors. Nested arrays have been utilized by Pal et al [42] to increase the number of virtual sensors and estimate more sources than the number of sensors. Qin et. al. [43] have developed a generalized scheme to generate a geometry based on co-prime array concept. In this method two linear sub arrays are used to generate a difference co-array. This approach increases the effective number of degrees of freedom for the direction of arrival estimation. Two concepts of coprime arrays are introduced, one is Coprime Array with Compressed Inter-element Spacing (CACIS) in which one of the array is compressed by an integer factor to reduce the interelement spacing of the array. Another structure studied is the Coprime Array with 
Displaced Sub-arrays (CADiS). This array structure indeed offer some advantages related to degrees of freedom for creating covariance matrix, however, this technique is currently limited to linear arrays.

The use of aperiodic geometries can reduce the problem of random aperture sampling significantly and make the layout practically realizable. Fractal geometries [45] have effectively been used in the design of aperiodic arrays. These studies, however, utilize classical array processing framework and much of the results may not be applicable to compressive sensing scenario. In [18], Spence et al. introduce a design technique to generate antenna array layouts from aperiodic tiling. The technique uses a slight perturbation in inflation technique to generate aperiodic tiling. They have used Genetic Algorithm optimization scheme to achieve low sidelobe levels and no grating lobes over wide bandwidths. This technique, however, is suitable for conventional array signal processing algorithms. Its aim is also somewhat different from what we intend. Our intention is to reduce the number of sensors in such a way that the compressed sensing algorithms like BPDN [12] generates more accurate results.

In this chapter, we device an optimization technique that generates an antennaarray layout through a disturbance of aperiodic tiling. This technique is well suited to any array design methodology that can be generated by transformations of a few initial points. These transformations include translation, rotation and scaling. The only design parameters for this optimization scheme is the position of the initial sets of points.

The rest of the chapter is organized as follows. In Section 5.2, we study different properties of the array manifold matrix or measurement matrix relevant to geometrical design. In Section 5.3, we discuss the array geometries. In Section 5.4, Penrose aperiodic tiling is discussed. Array geometry design based on inflation technique is 
discussed in Section 5.6. In Section 5.7, we develop an optimization technique to generate aperiodic antenna layout. In Section 5.9, we present results from performing simulations. Finally, in Section 5.10, we present concluding remarks.

\subsection{The measurement Matrix}

Suppose we have a number of narrow-band signals coming from $m$ directions and we have $L$ sensors in our array which are uniformly placed at a distance of $\lambda / 2$ from each other. $\lambda$ being the wavelength corresponding to the center frequency, $f_{c}$, of our receiver. These sensors form what is called a uniform linear array (ULA.) We assume that the signals are coming from any of the $N$ directions, in which we divide the DOA space. Then our received signal, $y$ is given by

$$
\boldsymbol{y}=\boldsymbol{A x} .
$$

The measurement matrix, $\boldsymbol{A}$, has a central importance to CS theory. Its properties are crucial to the exact recovery of sparse vectors from a very few observations. Ender [22] and Herman et. al. [21] have studied the problem of applying Compressed Sensing [20] to the problem of DOA estimation. Both have used Fourier matrix as the measurement matrix. They have taken random samples in either frequency [22] or in time domain [21]. Ender also suggested random sampling of aperture to generate measurement matrix. But placing sensors, having finite spacial span, into random pattern is not practicable. Here, we study the problem of radar target azimuth estimation focusing on the geometry aspect of the measurement matrix.

The problem model is the Single Measurement Vector model, as discussed pre- 
viously in Section 2.6, given by

$$
\boldsymbol{y}=\boldsymbol{A x}
$$

here

$$
\|\boldsymbol{x}\|_{0}=s
$$

Here, $s$ represents the sparsity or non-zero elements of the vector $\boldsymbol{x}$, which in our case would equal the number of sources, $K$.

One important property that the matrix $\boldsymbol{A}$ has to observe is the Restricted Isometry Property [13] given by 2.47 .

One of the few matrices that observe this property is the Fourier matrix [13]. Another significant parameter is the mutual coherence, $\mu$, which indicates the level of sparsity that can be recovered with very high probability.

For the system $\boldsymbol{y}=\boldsymbol{\Phi} \boldsymbol{\Psi} \boldsymbol{x}, \mu$ is defined by (2.43).

If the number of measurements, $M$, for S-sparse vector obeys the following inequality

$$
M \geq C \mu(\Phi, \Psi)^{2} S \log (N)
$$

for some positive constant, $C$, then the solution to this problem is exact with "overwhelming probability" [13].

One such $\Phi, \Psi$ pair with low $\mu$ is the Fourier matrix and identity matrix pair. In our case, i.e $y=A x$, where $\Phi=A, \Psi=I$. But we still have to take care of the RIP given by (2.47.) For this, the matrix A needs to have columns that are almost-orthogonal.

Suppose there is a matrix $\boldsymbol{B}=\boldsymbol{A}^{H} \boldsymbol{A}$, the diagonal entries of $\boldsymbol{B}$ must be unity as the vectors are normalized. The off-diagonal entries must be as close to zero as possible. This depends on the column vectors of matrix $\boldsymbol{A}$. For a regularly 
sampled aperture, as we would see, the CS results are not very promising. But for a randomly sampled aperture, CS algorithms perform much better. What we explore next is a kind of aperiodic geometry obtained by tiling of very simple base shapes. This aperiodic structure was discovered by Roger Penrose in 1970 and has since been found in naturally occurring crystalline materials as well [23]. This structure, being aperiodic, is naturally sparse (average sensor spacing is greater than $\lambda / 2$ ) as it doesn't contain any redundancies which are signature of periodic arrays like rectangular or its 1-D counterpart, uniform linear arrays. Other aperiodic arrays like fractal arrays have been thoroughly studied in the literature [24]. Spence and Werner [18] have studied the design of broadband array antennas using aperiodic structure. But this geometry hasn't been studied yet from the perspective of CS theory. Next we discuss the method to form array geometry from aperiodic tiling.

\subsection{Discussion Regarding Geometry}

In Chapter 4, the geometry used for azimuth estimation was Uniform Linear Ar$\operatorname{ray}(\mathrm{ULA})$ in $1-\mathrm{D}$. The distance between each element was set to $d=\lambda / 2$, where $\lambda=c / f_{\max }$. Here $c$ is the speed of light and $f_{\max }$ is the maximum frequency of the broad-band signal. In the case of uniform sampling of the array aperture, we run in to grating lobe issues for broadband signals. These grating lobes appear due to to the periodicity of the array geometry and are artifacts of spatial aliasing. But there are not many degrees of freedom when designing an array geometry in 1-D. In 2-D, we can have a lot of interesting geometries that can be explored to address different issues.

Here, we discuss a particular set of geometries based on aperiodic tiling. This 
type of geometry has some promise as these geometries are aperiodic and sparse. Aperiodicity is a trait that can be exploited to make the rows of the measurement matrix as much orthogonal as possible. Sparsity is also very desirable when we are working with compressive sensing.

The motivation is to find such geometry/set of geometries that are better suited (performance-wise) to algorithms developed for narrowband CS and broadband DOA estimation in this thesis.

\subsection{Penrose Tiling}

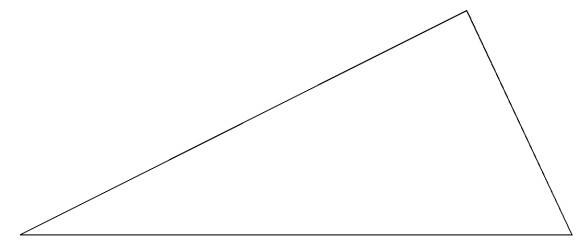

(a) Tile AL

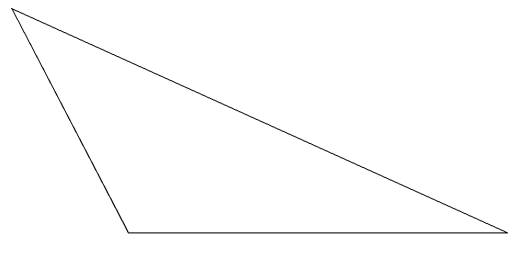

(b) Tile AS

Figure 5.1: Basic Penrose Tiles.

Penrose tiling consists of two base tiles $A L$ and $A S$, as shown in Figure 5.1. These two tiles can be used to fill any 2-D plane in an aperiodic fashion. There are two methods to do that. The first method is rule based. It starts from a single tile and based on edge matching rules builds tiles around the structure. This method can run into problems when the tiles can no more be placed seamlessly. The other method is called "inflation". In this method each tile is scaled up by a factor and is then sub-divided into the base tiles AL and AS. This process is repeated until the 
desired plane is completely filled.

\subsection{Danzer Tiling}

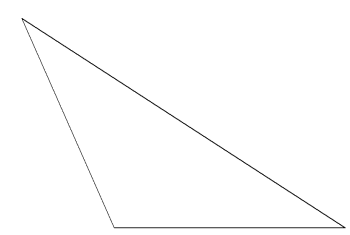

(a) Tile 1

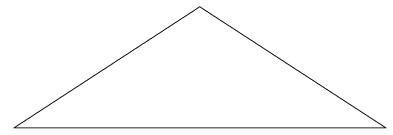

(b) Tile 2

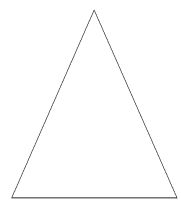

(c) Tile 3

Figure 5.2: Basic Danzer Tiles.

Danzer tiling consists of three base tiles type-1, type-2 and type-3, as shown in Figure 5.2. These three tiles can be used to fill any 2-D plane in an aperiodic fashion. There are two methods to do that. The first method is rule based. It starts from a single tile and based on edge matching rules builds tiles around the structure. This method can run into problems when the tiles can no more be placed seamlessly. The other method is called "inflation". In this method each tile is scaled up by a factor and is then sub-divided into the three base tiles. This process is repeated until the desired plane is completely filled. In the following section we discuss this method in detail for Penrose tiling and subsequently for Danzer tiling. 


\subsection{Array Formation Using Inflation}

In this section, we develop the mathematics behind the inflation process. This would be useful later on as we develop an optimization problem to solve the broad-band geometry problem.

We start from the two base triangles AL and AS. The first step is to find the 2-D co-ordinates of the indices of these triangles. Each 2-D point in space, $\boldsymbol{p}_{i}$ is defined by a $3 \times 1$ vector. The reason to represent a 2 -D point by a $3 \times 1$ vector is to incorporate translations along with rotations in our matrix transformation scheme. Next we describe the transformation matrices required for the transformation of base triangles to fill arbitrary 2-D plane.

\subsubsection{The Rotation Matrix}

The rotation matrix, $\boldsymbol{R}(\theta)$, rotates a vector, $\boldsymbol{v}$, by an angle $\theta$ to give a new vector,

$$
\boldsymbol{v}^{\prime}=\boldsymbol{R}(\theta) \boldsymbol{v}
$$

where,

$$
\boldsymbol{R}(\theta)=\left[\begin{array}{ccc}
\cos (\theta) & -\sin (\theta) & 0 \\
\sin (\theta) & \cos (\theta) & 0 \\
0 & 0 & 1
\end{array}\right]
$$

This is in fact the rotation 3-matrix representing a rotation along z-axis. 


\subsubsection{The Translation Matrix}

The matrix that translates a vector, $\boldsymbol{v}$, in $\mathrm{x}$ and $\mathrm{y}$ direction, to a new vector, $\boldsymbol{v}^{\prime}$, is given by, $\boldsymbol{T}(x, y)$. This means,

$$
\boldsymbol{v}^{\prime}=\boldsymbol{T}(x, y) \boldsymbol{v}
$$

where,

$$
\boldsymbol{T}(x, y)=\left[\begin{array}{lll}
0 & 0 & x \\
0 & 0 & y \\
0 & 0 & 1
\end{array}\right]
$$

\subsubsection{The Scaling Matrix}

The scaling matrix scales $\mathrm{x}$ and y coordinates of any vector $\boldsymbol{v}$ by transforming it to $\boldsymbol{v}^{\prime}$. That is,

$$
\boldsymbol{v}^{\prime}=\boldsymbol{S}(s x, s y) \boldsymbol{v}
$$

where, $s x$ and $s y$ are two scalars, giving $\mathrm{x}$ and $\mathrm{y}$ scaling respectively:

$$
\boldsymbol{S}(s x, s y)=\left[\begin{array}{ccc}
s x & 0 & 0 \\
0 & s y & 0 \\
0 & 0 & 1
\end{array}\right] .
$$

\subsubsection{The Flipping Matrix}

This matrix, $\boldsymbol{F}_{x}$, flips or inverts the x-coordinates of a vector $\boldsymbol{v}$ to give $\boldsymbol{v}^{\prime}$ :

$$
\boldsymbol{v}^{\prime}=\boldsymbol{F}_{x} \boldsymbol{v}
$$


where,

$$
\boldsymbol{F}_{x}=\left[\begin{array}{ccc}
-1 & 0 & 0 \\
0 & 1 & 0 \\
0 & 0 & 1
\end{array}\right]
$$

This matrix is in fact a scaling matrix with -1 and 1 set as $\mathrm{x}$ and $\mathrm{y}$ scaling parameters, respectively. It can also be written as

$$
\boldsymbol{F}_{x}=\boldsymbol{S}(-1,1)
$$

The translation, rotation and scaling matrices can be applied to a vector or set of vectors (matrix) by left multiplying the vector or the matrix by each transformation matrix in a sequential manner,

$$
v^{\prime}=\boldsymbol{S}_{k} \boldsymbol{R}_{l} \boldsymbol{T}_{m} \ldots \boldsymbol{S}_{1} \boldsymbol{R}_{1} \boldsymbol{T}_{1} v
$$

or by forming a single matrix and then left multiplying the vector or matrix by that matrix,

$$
v^{\prime}=\boldsymbol{T} v
$$

Here, $T$ represents the combined transformation matrix, given by

$$
\boldsymbol{T}=\boldsymbol{S}_{k} \boldsymbol{R}_{l} \boldsymbol{T}_{m} \ldots \boldsymbol{S}_{1} \boldsymbol{R}_{1} \boldsymbol{T}_{1}
$$

The sequence of multiplication is application dependent and would become apparent for our application soon.

Any triangle can be uniquely represented by 3 vertices $\boldsymbol{p}_{1}, \boldsymbol{p}_{2}, \boldsymbol{p}_{3}$. Here,

$$
\boldsymbol{p}_{i}=\left[\begin{array}{lll}
p_{i x} & p_{i y} & 1
\end{array}\right]^{T} .
$$

By setting the third component of all the vectors to 1, we can perform translations, scalings and rotations using 3-matrices and then dropping or ignoring the third component of the resultant vector $\boldsymbol{v}^{\prime}$. 
Since we have built the mathematical foundation for inflation process, we can now move to the application of inflation process on Penrose tiling.

\subsubsection{Penrose Tiling Using Inflation Process}

Penrose triangles are represented by two edge lengths $a$ and $b$ and an angle $\theta=\frac{\pi}{5}$. Setting $a=1, b$ equals inverse of the golden ratio, 1.6180 . That is, $b=0.6180$.

Tile $A L$ is represented by the following three vertices:

$$
\begin{aligned}
p^{A L} & =\left[\begin{array}{lll}
\left(p_{1}^{A L}\right)^{T} & \left(p_{2}^{A L}\right)^{T} & \left(p_{3}^{A L}\right)^{T}
\end{array}\right]^{T}, \\
p_{1}^{A L} & =\left[\begin{array}{lll}
0 & 0 & 1
\end{array}\right]^{T}, \\
p_{2}^{A L} & =\left[\begin{array}{lll}
a & 0 & 1
\end{array}\right]^{T}, \\
p_{3}^{A L} & =\left[\begin{array}{lll}
a-b \cos (-2 \theta) & -b \sin (-2 \theta) & 1
\end{array}\right]^{T} .
\end{aligned}
$$

Tile $A S$ is represented by the following three vertices:

$$
\begin{aligned}
& p^{A S}=\left[\begin{array}{lll}
\left(p_{1}^{A S}\right)^{T} & \left(p_{2}^{A S}\right)^{T} & \left(p_{3}^{A S}\right)^{T}
\end{array}\right]^{T}, \\
& p_{1}^{A S}=\left[\begin{array}{lll}
0 & 0 & 1
\end{array}\right]^{T}, \\
& p_{2}^{A S}=\left[\begin{array}{lll}
a & 0 & 1
\end{array}\right]^{T}, \\
& p_{3}^{A S}=\left[\begin{array}{lll}
-b \cos (-2 \theta) & -b \sin (-2 \theta) & 1
\end{array}\right]^{T} \text {. }
\end{aligned}
$$

The Level I Penrose triangle, $A L_{I}$ is shown in Figure 5.4a. It consists of two $A L$ and one $A S$ base triangles. The triangle, $A L_{I}$, is represented by 9 vectors, 3 vectors for each triangle.

The transformation matrices for each triangle are given as follows. 


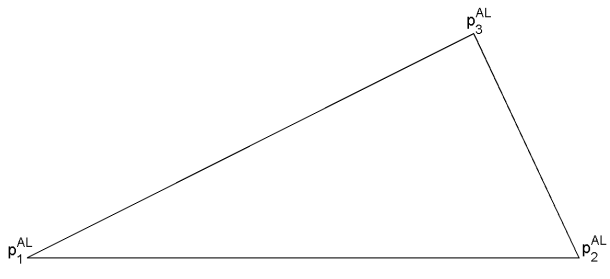

(a) Tile AL

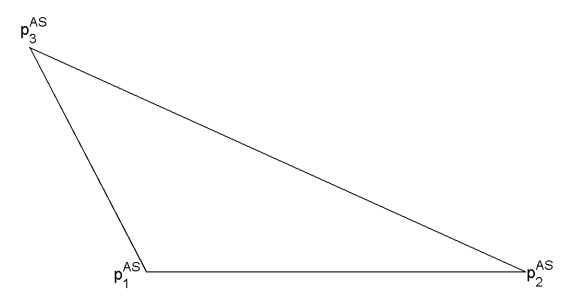

(b) Tile AS

Figure 5.3: Basic Penrose Tiles.

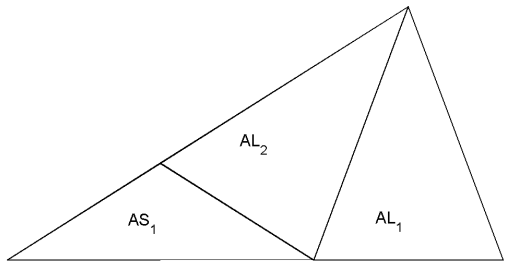

(a) Tile AL

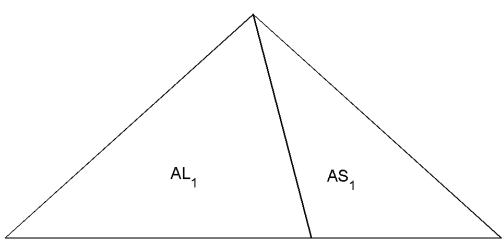

(b) Tile AS

Figure 5.4: Level I Penrose Tiles. 


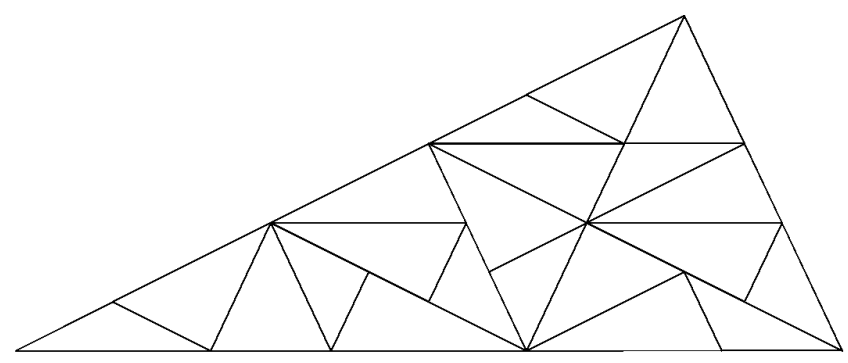

Figure 5.5: Level III Penrose Tile AL.

Transformation matrix for triangle $A L_{1}$ :

$$
\boldsymbol{T}_{A L_{1}}=\boldsymbol{S}(s x, s y) \boldsymbol{T}(a-b \cos (-\theta),-b \sin (-\theta)) \boldsymbol{R}(-4 \theta) \boldsymbol{T}(-a, 0) .
$$

Transformation matrix for triangle $A L_{2}$ :

$$
\boldsymbol{T}_{A L_{2}}=\boldsymbol{S}(s x, s y) \boldsymbol{T}(a, 0) \boldsymbol{R}(-3 \theta) \boldsymbol{T}(-a, 0) .
$$

Transformation matrix for triangle $A S_{1}$ :

$$
\boldsymbol{T}_{A S_{1}}=\boldsymbol{S}(s x, s y) \boldsymbol{T}(-b \cos (-4 \theta),-b \sin (-4 \theta)) \boldsymbol{R}(-4 \theta) .
$$

Here, $s x=s y=\frac{a+b}{a}$.

The complete transformation matrix that forms Level I Penrose triangle, $A L_{I}$, is given by:

$$
\boldsymbol{P}_{A L_{I}}=\boldsymbol{T}_{A L_{I}}\left[\begin{array}{lll}
\left(p^{A L}\right)^{T} & \left(p^{A L}\right)^{T} \quad\left(p^{A S}\right)^{T}
\end{array}\right]^{T}
$$

Here the matrix, $\boldsymbol{T}_{A L_{I}}$, is built from the transformation matrices $\boldsymbol{T}_{A L_{1}}, \boldsymbol{T}_{A L_{2}}$, 
$\boldsymbol{T}_{A S_{1}}$, in the following manner.

$$
\boldsymbol{T}_{A L_{I}}=\left[\begin{array}{ccccccccc}
\boldsymbol{T}_{A L_{1}} & 0 & 0 & 0 & 0 & 0 & 0 & 0 & 0 \\
0 & \boldsymbol{T}_{A L_{1}} & 0 & 0 & 0 & 0 & 0 & 0 & 0 \\
0 & 0 & \boldsymbol{T}_{A L_{1}} & 0 & 0 & 0 & 0 & 0 & 0 \\
0 & 0 & 0 & \boldsymbol{T}_{A L_{2}} & 0 & 0 & 0 & 0 & 0 \\
0 & 0 & 0 & 0 & \boldsymbol{T}_{A L_{2}} & 0 & 0 & 0 & 0 \\
0 & 0 & 0 & 0 & 0 & \boldsymbol{T}_{A L_{2}} & 0 & 0 & 0 \\
0 & 0 & 0 & 0 & 0 & 0 & \boldsymbol{T}_{A S_{1}} & 0 & 0 \\
0 & 0 & 0 & 0 & 0 & 0 & 0 & \boldsymbol{T}_{A S_{1}} & 0 \\
0 & 0 & 0 & 0 & 0 & 0 & 0 & 0 & \boldsymbol{T}_{A S_{1}}
\end{array}\right] .
$$

Note that $0=0^{3 \times 3}$, is a $3 \times 3$ matrix with all entires set to zero.

Similar transformation can be derived for Level I Penrose Triangle, $A S_{I} . A S_{I}$ is shown in Figure 5.4b. It constists of one $A L$ and one $A S$ triangle.

The transformation matrices for each of the triangles are given as follows.

Transformation matrix for triangle $A L_{1}$ :

$$
\boldsymbol{T}_{A L_{1}}=\boldsymbol{S}(s x, s y) \boldsymbol{R}(4 \theta) \boldsymbol{T}(b \cos (-4 \theta), b \sin (-4 \theta))
$$

Transformation matrix for triangle $A S_{1}$ :

$$
\boldsymbol{T}_{A S_{1}}=\boldsymbol{S}(s x, s y) \boldsymbol{R}(4 \theta) \boldsymbol{T}(a+b \cos (-4 \theta), b \sin (-4 \theta)) .
$$

Here, $s x=s y=\frac{a+b}{a}$, as before.

The complete transformation matrix that forms Level I Penrose Triangle, $A S_{I}$, is given by:

$$
\boldsymbol{P}_{A S_{I}}=\boldsymbol{T}_{A S_{I}}\left[\begin{array}{ll}
\left(p^{A L}\right)^{T} & \left(p^{A S}\right)^{T}
\end{array}\right]^{T}
$$


Here the matrix, $\boldsymbol{T}_{A S_{I}}$, is built from the transformation matrices $\boldsymbol{T}_{A L_{1}}, \boldsymbol{T}_{A S_{1}}$, in the following manner.

$$
\boldsymbol{T}_{A S_{I}}=\left[\begin{array}{cccccc}
\boldsymbol{T}_{A L_{1}} & 0 & 0 & 0 & 0 & 0 \\
0 & \boldsymbol{T}_{A L_{1}} & 0 & 0 & 0 & 0 \\
0 & 0 & \boldsymbol{T}_{A L_{1}} & 0 & 0 & 0 \\
0 & 0 & 0 & \boldsymbol{T}_{A S_{1}} & 0 & 0 \\
0 & 0 & 0 & 0 & \boldsymbol{T}_{A S_{1}} & 0 \\
0 & 0 & 0 & 0 & 0 & \boldsymbol{T}_{A S_{1}}
\end{array}\right] .
$$

Note that $0=0^{3 \times 3}$, is a $3 \times 3$ matrix with all entires set to zero. Using these transformation matrices we can get the Level-k matrix for each tile in the following manner:

$$
\begin{aligned}
& \boldsymbol{T}_{A L_{k}}=\boldsymbol{T}_{A L_{k-1}} \ldots \boldsymbol{T}_{A L_{I} I} \boldsymbol{T}_{A L_{I}} \\
& \boldsymbol{T}_{A S_{k}}=\boldsymbol{T}_{A S_{k-1} \ldots \boldsymbol{T}_{A S_{I} I} \boldsymbol{T}_{A S_{I}}}
\end{aligned}
$$

\subsubsection{Danzer Tiling Using Inflation Process}

Danzer tiles (triangles) are represented by three edge lengths $a, b$ and $c$ and an angle $\theta=\frac{\pi}{7}$. Setting $a=1, b$ and $c$ are given by:

$$
\begin{aligned}
& b=a \frac{\sin (2 \theta)}{\sin (\theta)}, \\
& c=a \frac{\sin (4 \theta)}{\sin (\theta)} .
\end{aligned}
$$




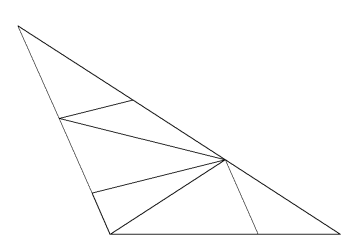

(a) Tile 1

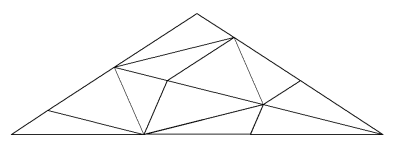

(b) Tile 2

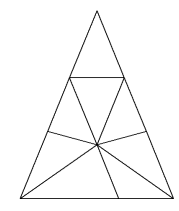

(c) Tile 3

Figure 5.6: Level I Danzer Tiles.

Tile $D_{1}$ (Figure 5.2a) is represented by the following three vertices.

$$
\begin{aligned}
p^{D_{1}} & =\left[\begin{array}{lll}
\left(p_{1}^{D_{1}}\right)^{T} & \left(p_{2}^{D_{1}}\right)^{T} & \left(p_{3}^{D_{1}}\right)^{T}
\end{array}\right]^{T}, \\
p_{1}^{D_{1}} & =\left[\begin{array}{lll}
0 & 0 & 1
\end{array}\right]^{T}, \\
p_{2}^{D_{1}} & =\left[\begin{array}{lll}
a & 0 & 1
\end{array}\right]^{T}, \\
p_{3}^{D_{1}} & =\left[\begin{array}{lll}
b \cos (4 \theta) & b \sin (4 \theta) & 1
\end{array}\right]^{T} .
\end{aligned}
$$

Tile $D_{2}$ (Figure 5.2b) is represented by the following three vertices.

$$
\begin{aligned}
p^{D_{2}} & =\left[\begin{array}{lll}
\left(p_{1}^{D_{2}}\right)^{T} & \left(p_{2}^{D_{2}}\right)^{T} & \left(p_{3}^{D_{2}}\right)^{T}
\end{array}\right]^{T}, \\
p_{1}^{D_{2}} & =\left[\begin{array}{lll}
0 & 0 & 1
\end{array}\right]^{T}, \\
p_{2}^{D_{2}} & =\left[\begin{array}{lll}
c & 0 & 1
\end{array}\right]^{T}, \\
p_{3}^{D_{2}} & =\left[\begin{array}{lll}
b \cos (2 \theta) & b \sin (2 \theta) & 1
\end{array}\right]^{T} .
\end{aligned}
$$


Tile $D_{3}$ (Figure 5.2c) is represented by the following three vertices.

$$
\begin{aligned}
p^{D_{3}} & =\left[\begin{array}{lll}
\left(p_{1}^{D_{3}}\right)^{T} & \left(p_{2}^{D_{3}}\right)^{T} & \left(p_{3}^{D_{3}}\right)^{T}
\end{array}\right]^{T}, \\
p_{1}^{D_{3}} & =\left[\begin{array}{lll}
0 & 0 & 1
\end{array}\right]^{T}, \\
p_{2}^{D_{3}} & =\left[\begin{array}{lll}
a & 0 & 1
\end{array}\right]^{T}, \\
p_{3}^{D_{3}} & =\left[\begin{array}{lll}
c \cos (3 \theta) & c \sin (3 \theta) & 1
\end{array}\right]^{T} .
\end{aligned}
$$

The Level I Danzer triangle, $D_{1 I}$ is shown in Fig 5.6a. It consists of three $D_{1}$, two $D_{2}$ and one $D_{3}$ base triangles. The triangle, $D_{1 I}$, is represented by 18 vectors, 3 vectors for each triangle.

The transformation matrices for each triangle are given as follows.

Transformation matrix for triangle $D_{21}$ :

$$
\boldsymbol{T}_{D_{21}}=\boldsymbol{S}(s x, s y) \boldsymbol{R}(-5 \theta) \boldsymbol{F}_{\boldsymbol{x}}
$$

Transformation matrix for triangle $D_{11}$ :

$$
\boldsymbol{T}_{D_{11}}=\boldsymbol{S}(s x, s y) \boldsymbol{T}(b, 0) .
$$

Transformation matrix for triangle $D_{12}$ :

$$
\boldsymbol{T}_{D_{12}}=\boldsymbol{S}(s x, s y) \boldsymbol{T}(a \cos (4 \theta), a \sin (-4 \theta)) \boldsymbol{R}(11 \theta) .
$$

Transformation matrix for triangle $D_{22}$ :

$$
\boldsymbol{T}_{D_{22}}=\boldsymbol{S}(s x, s y) \boldsymbol{T}((a+b) \cos (4 \theta),(a+b) \sin (4 \theta)) \boldsymbol{R}(-8 \theta) \boldsymbol{F}_{\boldsymbol{x}} .
$$

Transformation matrix for triangle $D_{13}$ :

$\boldsymbol{T}_{D_{13}}=\boldsymbol{S}(s x, s y) \boldsymbol{T}(a \cos (\theta), a \sin (\theta)) \boldsymbol{T}((a+b) \cos (4 \theta),(a+b) \sin (4 \theta)) \boldsymbol{R}(-6 \theta)$. 
Transformation matrix for triangle $D_{31}$ :

$$
\boldsymbol{T}_{D_{31}}=\boldsymbol{S}(s x, s y) \boldsymbol{T}((a+b) \cos (4 \theta),(a+b) \sin (4 \theta)) \boldsymbol{R}(\theta) .
$$

The complete transformation matrix that forms Level I Danzer triangle, $D_{1 I}$ is given by:

$$
\boldsymbol{P}_{A L_{I}}=\boldsymbol{T}_{A L_{I}}\left[\begin{array}{ll}
\left(p^{A L}\right)^{T} & \left(p^{A L}\right)^{T} \quad\left(p^{A S}\right)^{T}
\end{array}\right]^{T}
$$

To form an antenna array based on this geometry, we can place individual antenna sensors at the indices of each triangle as shown in Figure 5.7.

\subsection{Optimization Scheme/Algorithm}

The measurement matrix $\boldsymbol{A}$ contains an exponential at row $k$, and column $l$. That is,

$$
\boldsymbol{A}(k, l)=e^{j \kappa_{0} \boldsymbol{p}_{k}^{T} \boldsymbol{r}_{l}}, k=\{1, \ldots, M\}, l=\{1, \ldots, N\}
$$

where $\boldsymbol{r}_{l}$ is the normalized direction vector for direction $l$ and $\boldsymbol{p}_{k}$ is the position vector for sensor $k . \kappa_{0}=\frac{2 \pi f_{0}}{c}$. Or we could write $\boldsymbol{A}$ as $\boldsymbol{A}=e^{j \kappa_{0} \boldsymbol{P}^{T} \boldsymbol{R}}$.

Matrix $\boldsymbol{B}$ can be written as

$$
\boldsymbol{B}(u, v)=\sum_{\xi=1}^{M} e^{j \kappa_{0}\left(\boldsymbol{p}_{\xi}^{T} \boldsymbol{r}_{v}-\boldsymbol{p}_{\xi}^{T} \boldsymbol{r}_{u}\right)} u, v \in\{1, \ldots, N\} .
$$

There are $N^{2}$ entries in matrix $\boldsymbol{B}$.

$\boldsymbol{P}$ is a $3 \times M$ matrix containing position 3 -vectors of $M$ sensors as its columns. We choose three points $\boldsymbol{p}_{0}, \boldsymbol{p}_{1}, \boldsymbol{p}_{2}$ inside Danzer triangles of Type-I, II and III, respectively. These initial nine points (three points for each tile) pass through a 


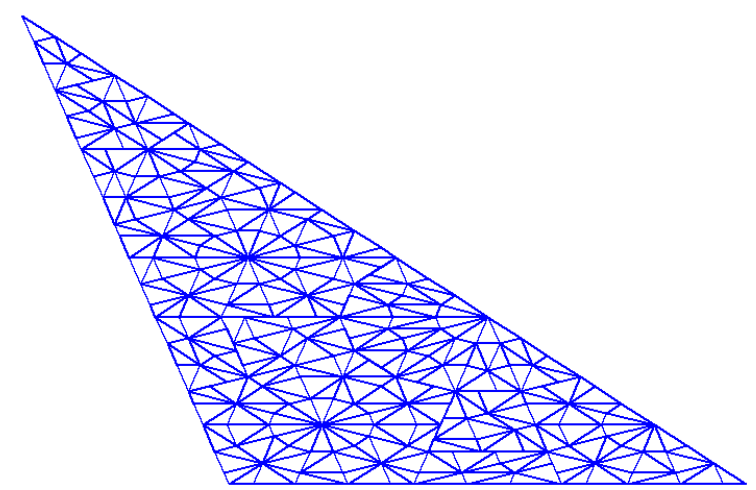

a)

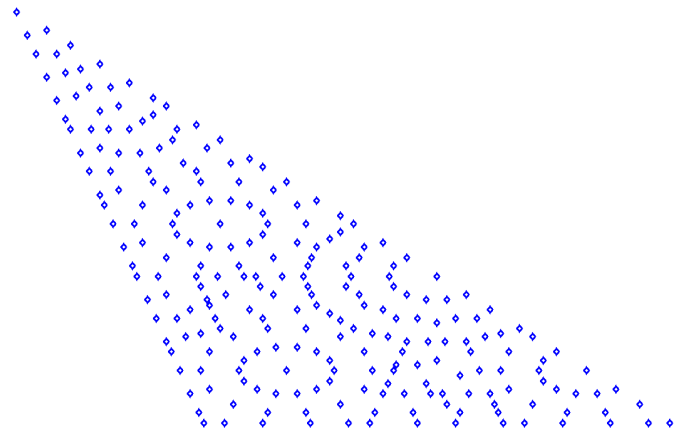

b)

Figure 5.7: a) Level-3 Danzer aperiodic tiling b) Sensors array antenna formed by placing sensors at the indices of each triangle. 
transformation matrix involving translation, rotation and scaling sub matrices to form the next level Danzer triangle. Now $\boldsymbol{P}=\boldsymbol{T} \boldsymbol{p}$, where $\boldsymbol{p}=\left[\begin{array}{lll}\boldsymbol{p}_{0}^{T} & \boldsymbol{p}_{1}^{T} & \boldsymbol{p}_{2}^{T}\end{array}\right]^{T}$.

We pose the following optimization problem:

$$
\begin{array}{ll}
\underset{\boldsymbol{p}}{\operatorname{minimize}} & \|\boldsymbol{B}-\mu \boldsymbol{I}\|_{2} \\
\text { subject to } & \boldsymbol{M}_{u} \boldsymbol{p}_{u}+\boldsymbol{C}_{u} \leq 0 .
\end{array}
$$

Here, $\boldsymbol{M}_{u}$ and $\boldsymbol{C}_{u}$ contains slopes and y-intercepts, respectively, of the base triangle boundaries. The inequality would constrain the initial points to lie on the boundary or inside the base triangles.

In its current form, this problem does not seem to be solvable. What we do next would pave the way for finding a solution to this problem.

Our objective is to bring $\boldsymbol{B}$ as close as possible to $\mu \boldsymbol{I}$, subject to certain conditions. There are $N^{2}$ equations to satisfy, apart from the inequality conditions on $p$. Each equation looks like:

$$
\begin{cases}\sum_{\xi=1}^{M} e^{j \kappa_{0}\left(\boldsymbol{T} \boldsymbol{p}_{\xi}^{T} \boldsymbol{r}_{v}-\boldsymbol{T} \boldsymbol{p}_{\xi}^{T} \boldsymbol{r}_{u}\right)}=\boldsymbol{\mu} & \text { if } u=v \\ \sum_{\xi=1}^{M} e^{j \kappa_{0}\left(\boldsymbol{T} \boldsymbol{p}_{\xi}^{T} \boldsymbol{r}_{v}-\boldsymbol{T} \boldsymbol{p}_{\xi}^{T} \boldsymbol{r}_{u}\right)}=0 & \text { otherwise. }\end{cases}
$$

When $u=v$, we have diagonal entries and the equations are:

$$
\sum_{\xi=1}^{M} e^{j \kappa_{0} 0}=\boldsymbol{\mu} .
$$

This condition can be satisfied if we multiply each column of $\boldsymbol{A}$ by $\frac{\mu}{M}$. When $u \neq v$, we have $N(N-1)$ equations:

$$
\sum_{\xi=1}^{M} e^{j \kappa_{0}\left(\boldsymbol{T} \boldsymbol{p}_{\xi}^{T} \boldsymbol{r}_{v}-\boldsymbol{T} \boldsymbol{p}_{\xi}^{T} \boldsymbol{r}_{u}\right)}=0 \quad u \neq v .
$$

For a particular $u$ and $v, u \neq v$, the equation in expanded form is

$$
e^{j \kappa_{0}\left([\boldsymbol{T} \boldsymbol{p}]_{1}^{T}\left(\boldsymbol{r}_{v}-\boldsymbol{r}_{u}\right)\right)}+e^{j \kappa_{0}\left([\boldsymbol{T} \boldsymbol{p}]_{2}^{T}\left(\boldsymbol{r}_{v}-\boldsymbol{r}_{u}\right)\right)}+\ldots+e^{j \kappa_{0}\left([\boldsymbol{T} \boldsymbol{p}]_{N}^{T}\left(\boldsymbol{r}_{v}-\boldsymbol{r}_{u}\right)\right)}=0 .
$$


We can choose the arguments of exponentials (on a unit circle) in such a way that their sum becomes equal to zero

$$
e^{j \epsilon_{1}}+e^{j \epsilon_{2}}+\ldots+e^{j \epsilon_{N}}=0
$$

Then,

$$
\begin{aligned}
{[\boldsymbol{T} \boldsymbol{p}]_{1}^{T}\left(\boldsymbol{r}_{v}-\boldsymbol{r}_{u}\right) } & =\frac{\epsilon_{1}}{\kappa_{0}} \\
{[\boldsymbol{T} \boldsymbol{p}]_{2}^{T}\left(\boldsymbol{r}_{v}-\boldsymbol{r}_{u}\right) } & =\frac{\epsilon_{2}}{\kappa_{0}} \\
& \cdot \\
& \cdot \\
{[\boldsymbol{T p}]_{M}^{T}\left(\boldsymbol{r}_{v}-\boldsymbol{r}_{u}\right) } & =\frac{\epsilon_{M}}{\kappa_{0}}
\end{aligned}
$$

There are $M$ equations for each $u, v$ pair. As $[\boldsymbol{T} \boldsymbol{p}]_{i}^{T}\left(\boldsymbol{r}_{v}-\boldsymbol{r}_{u}\right)$ is a scalar, we can transpose it to get $\left(\boldsymbol{r}_{v}-\boldsymbol{r}_{u}\right)^{T}[\boldsymbol{T} \boldsymbol{p}]_{i}$ or equivalently $\left(\boldsymbol{r}_{v}-\boldsymbol{r}_{u}\right)^{T}[\boldsymbol{T}]_{i} \boldsymbol{p}$, as the initial points denoted by p remain constant, and only the transformation matrix changes. As we have $M$ such equations, we can write them in a matrix form

$$
\hat{\boldsymbol{T}}_{u, v} \boldsymbol{p}=\epsilon,
$$

where, $\hat{\boldsymbol{T}}_{u, v}=\left(\boldsymbol{r}_{v}-\boldsymbol{r}_{u}\right)^{T} \boldsymbol{T}$ and $\epsilon=\left[\frac{\epsilon_{1}}{\kappa_{0}} \ldots \frac{\epsilon_{N}}{\kappa_{0}}\right]^{T}$.

We have $M^{2}-M$ equations of the form $\hat{\boldsymbol{T}}_{u, v} \boldsymbol{p}=\epsilon$ for each $u, v=\{1,2,3, \ldots, N\}, u \neq$ $v$.

All the combined equations could be written into one huge matrix equation

$$
\Upsilon p=E
$$


Here $\boldsymbol{\Upsilon}=\left[\begin{array}{c}\hat{\boldsymbol{T}}_{1,2} \\ \cdot \\ \cdot \\ \hat{\boldsymbol{T}}_{1, M-1} \\ \hat{\boldsymbol{T}}_{2,1} \\ \cdot \\ \cdot \\ \cdot \\ \cdot \\ \hat{\boldsymbol{T}}_{M, M-1}\end{array}\right], \boldsymbol{E}=\left[\boldsymbol{\epsilon}^{T} \boldsymbol{\epsilon}^{T} \ldots \boldsymbol{\epsilon}^{T}\right]^{T}$.

The optimization problem now becomes (constrained least-squared optimization problem):

$$
\begin{array}{cl}
\underset{\boldsymbol{p}}{\operatorname{minimize}} & \|\boldsymbol{\Upsilon} \boldsymbol{p}-\boldsymbol{E}\|_{2} \\
\text { subject to } & \boldsymbol{M}_{u} \boldsymbol{p}+\boldsymbol{C}_{u} \leq 0 .
\end{array}
$$

\subsection{Boundary Constraints for the Optimization Problem}

In this section we will discuss the boundary constraints for the optimization problem. The boundary constraints are given by the following inequality:

$$
\boldsymbol{M}_{u} \boldsymbol{p}_{u}+\boldsymbol{C}_{u} \leq 0
$$




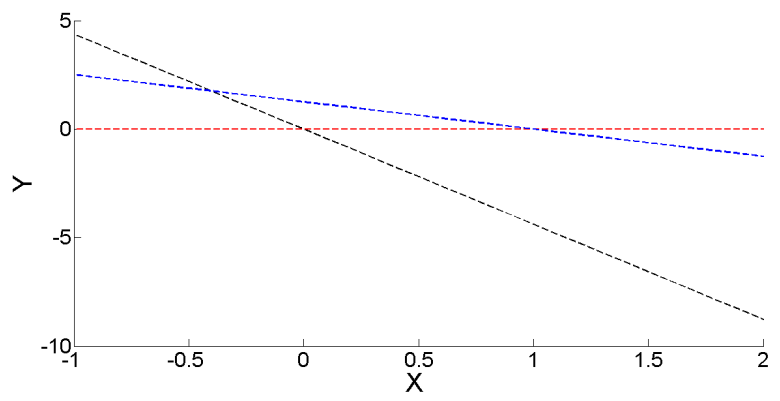

(a)

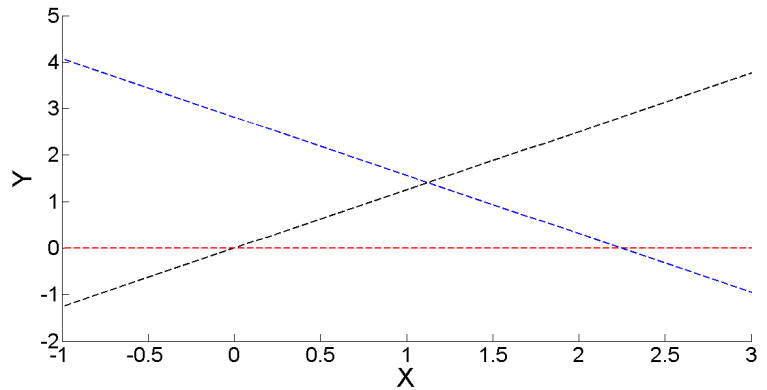

(b)

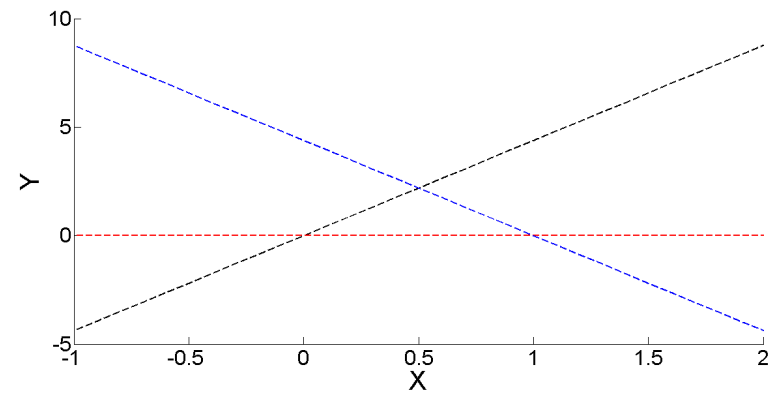

(c)

Figure 5.8: Optimization constraints depicted by lines for a) Danzer Tile 1, b) Danzer Tile 2, c) Danzer Tile 3. 


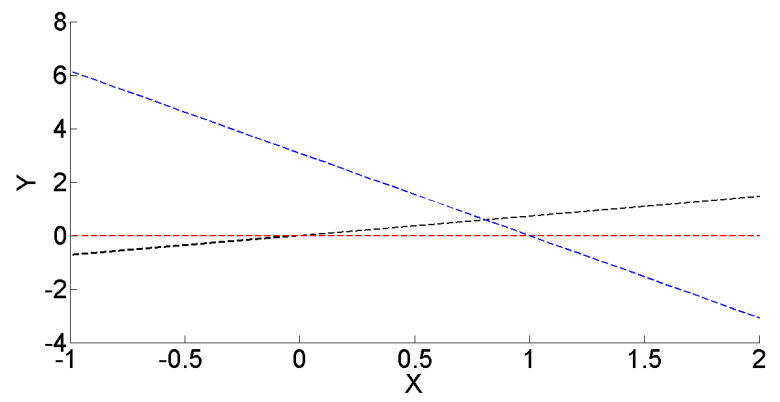

(a)

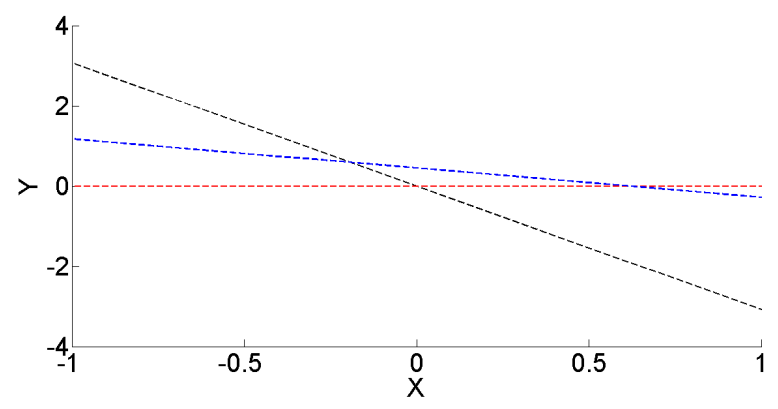

(b)

Figure 5.9: Optimization constraints depicted by lines for a) Penrose Tile AL, b) Penrose Tile AS. 
This inequality ensures that the solution lies inside or on the triangle boundaries. Matrix $\boldsymbol{M}$ is a $3 \times 2$ matrix. The first column of this matrix contains the slopes of the three edges of the triangles. The second column contains a value of 1 or -1 depending on whether we want to incude points above or below this line. The following equation describes an equation of the line:

$$
y=m x+c
$$

$m$ is a scalar defining the slope of the line, while $c$ defines $y$-intercept of the line. This formula is called "slope-intercept" form. We can write this formula in the following manner:

$$
y-m x-c \leq 0 \text {. }
$$

This equations gives all the $(x, y)$ pairs below the line defined by the slope-intercept pair $(m, c)$. Similarly,

$$
y-m x-c \geq 0
$$

gives us all the $(x, y)$ points that are above the line defined by the slope-intercept pair $(m, c)$

To define points lying inside a triangle, we have to use both (5.71) and (5.72). For consistency, and to define our optimization problem properly, we combine both these equations in a matrix form:

$$
M p+C \leq 0
$$

here, $\boldsymbol{M}=\left[\begin{array}{ll}-m & 1\end{array}\right]$, when the interior of the triangle lies below the line, or $\boldsymbol{M}=\left[\begin{array}{ll}m & -1\end{array}\right]$, when the interior of the triangle lies above the line defined by $(m, c) . \quad \boldsymbol{p}$ is a 2-vector, given by $\boldsymbol{p}=\left[\begin{array}{ll}x & y\end{array}\right]^{T}$. Similarly, $C=-c$, when the interior of the triangle lies below the line and $C=c$, when it lies above. 
For the complete set of conditions, we have the inequality:

$$
M p+C \leq 0
$$

where, $M$ is a $3 \times 2$ matrix formed by stacking the row-vectors for each boundary on top of each other. $C$ is a $3 \times 1$ vector, containing the three y-intercept values.

Figure 5.8 shows the boundary conditions for the three base tiles of Danzer geometry. We will be using these conditions when dealing with optimization for Danzer geometry. Figure 5.9 shows the boundary conditions for the two Penrose tiles.

For Danzer geometries, we have 9 constraints and the matrix $\boldsymbol{M}$ is of dimension $9 \times 2$ and matrix $\boldsymbol{C}$ has dimension $9 \times 1$. For Penrose geometry, due to the fact that there are only two base triangles, we have 6 constraints and as a result the matrix $\boldsymbol{M}$ is of dimension $6 \times 2$ and matrix $\boldsymbol{C}$ has dimension $6 \times 1$.

\subsection{Results}

In this section, we provide analysis of the two types of aperiodic geometries discussed in the previous part of this chapter; Danzer and Penrose. Algorithm 6 provides a summary of the step by step process of generation optimum geometries for each type. Using this process we generate an optimal geometry for both Danzer and Penrose tiling. These geometries are compared against random and rectangular grid geometries to study the merits of the approach taken in this Chapter.

There are three types of base tiles in Danzer geometry: Type-I, Type-II and Type-III. Each tile can be subdivided into sub-tiles using inflation process, explained 


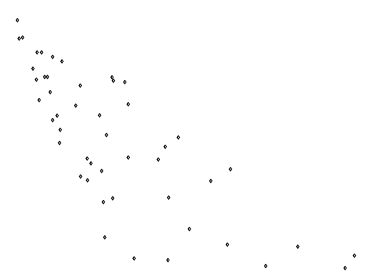

(a)

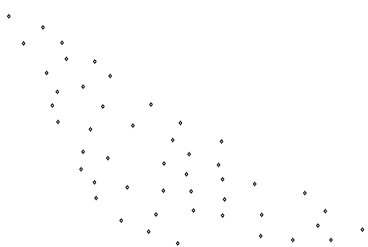

(b)

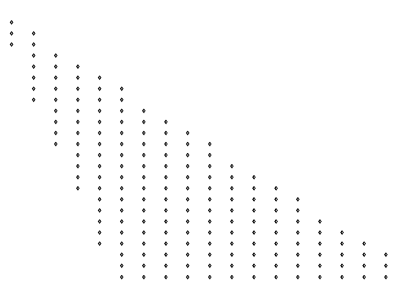

(c)

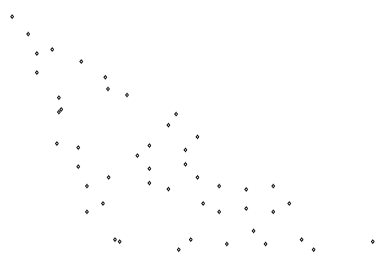

(d)

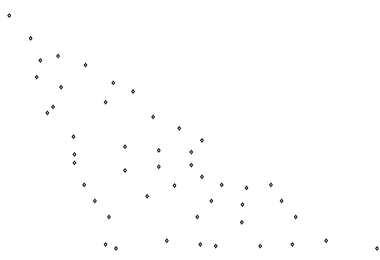

(e)

Figure 5.10: a) 48-element random array, b) 48-element constrained random array, c) 94-element triangular array (rectangular grid sampling), d) 48-element Danzer array, e) 48-element optimized Danzer array. 


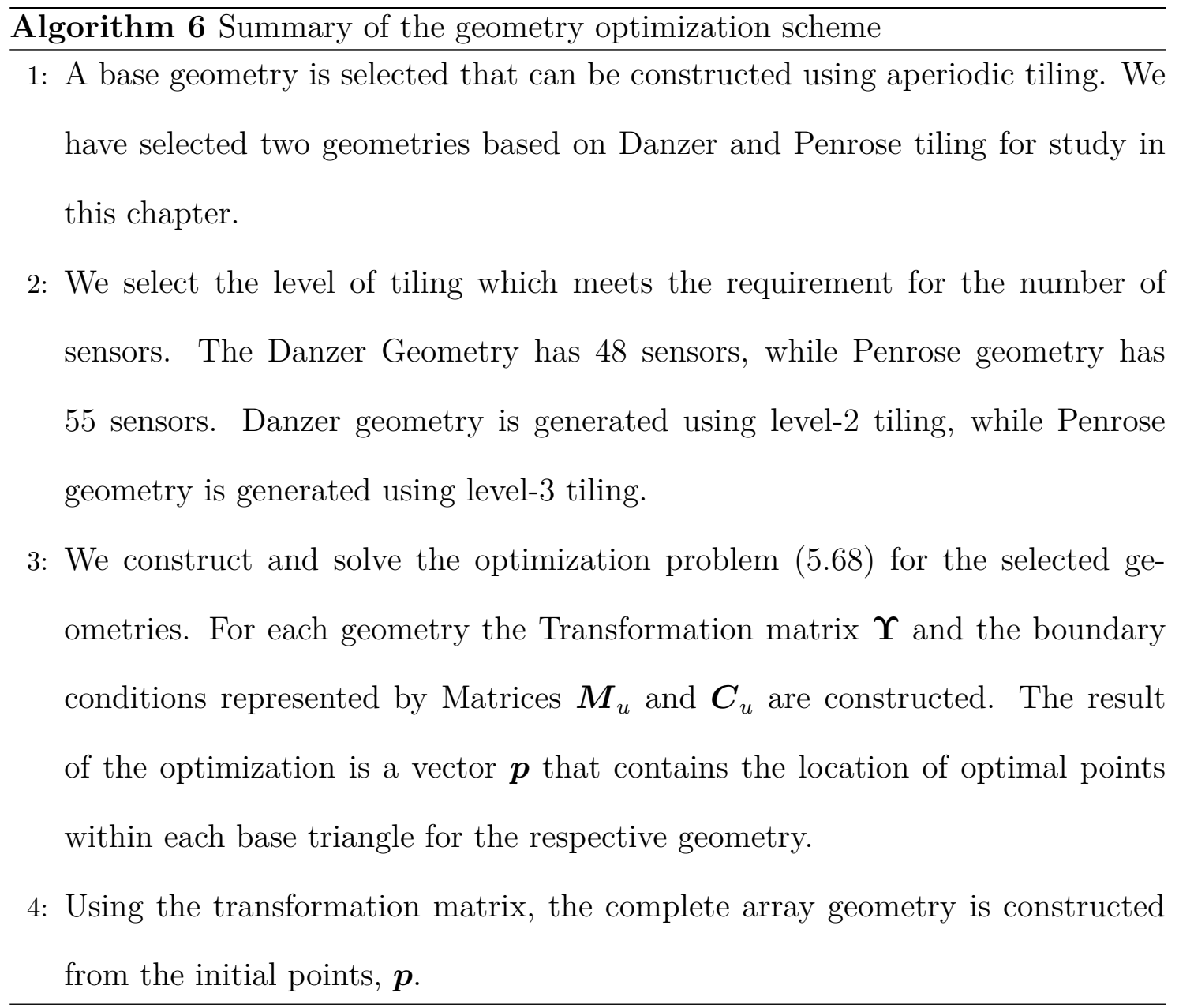

earlier in this chapter. Type-I level-1 tile (Figure 5.2a) contains only 6 sub-tiles. Type-II level-1 tile (Figure 5.2b) contains 11 sub-tiles, while, Type-III level-1 tile (Figure 5.2c) contains 7 sub-tiles. We can use any one of these tiles for our study in this section. Each sub-tile would contain one sensor. So total number of sensors contained in each tile equals the number of sub-tiles that constitues that particular tile. For a 2-D array of sensors, the number of senors (6,11 or 7) for level-1 tiles are not enough to resolve sources that are located in a close proximity. We have no option but to choose a level-2 tile. To keep the computation time of our simulations 
reasonable, we have chosen Danzer level-2, type-I triangle as the base geometry. It has the least number of sensors/elements for any type of Danzer tiles in level-2. There are $M=48$ elements in this array. We can see the array geometry in Figure $5.10 \mathrm{~d}$. After optimizing the array geometry according to the optimization problem given by (5.68) and summarized in Algorithm 6, we get the array shown in Figure 5.10e. We compare these geometries with three other given in Figures 5.10 (a, b and c). Figure 5.10a shows an array of 48 elements, whose positions were chosen randomly, but with a constraint that no two elements are closer to each other than $\lambda / 3$. This constraint was chosen to keep the practical difficulties of designing arrays that are too close to each other (a completely random array generated without any constraint would almost always have some elements very close together which isn't practically realizable.) Another geometry, which is very common, is the rectangular grid array, but its aperture has been chosen to be triangular to match with the one chosen for Danzer array. It is shown in Figures 5.10c.

Figure 5.11 shows the absolute of correlation matrix $A A^{H}$ for four array configurations. Although this matrix is different from matrix $\boldsymbol{B}(5.59)$ but it gives some insight into the mutual coherence properties of each array configuration. For random array configuration, the correlation matrix is very close to identity matrix. For rectangular grid configurations we see a lot of high valued off-diagonal entries which indicates that this configuration is not suitable for use with compressive sensing algorithms. For optimal array configuration, the off diagonal values are not much pronounced and are comparable to that of random array configuration.

Another type of aperiodic tiling scheme discussed in Section 5.4 is called Penrose tiling. We use level-2 tile $A S$, as it has 55 sub-tiles, comparable to the Danzer 


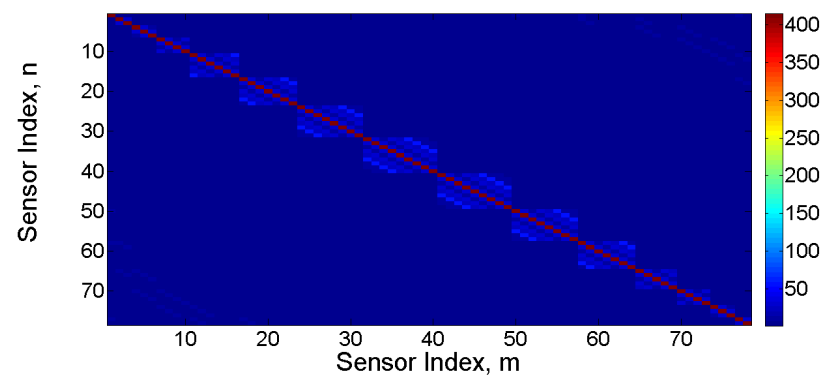

(a) Rectangular-grid array

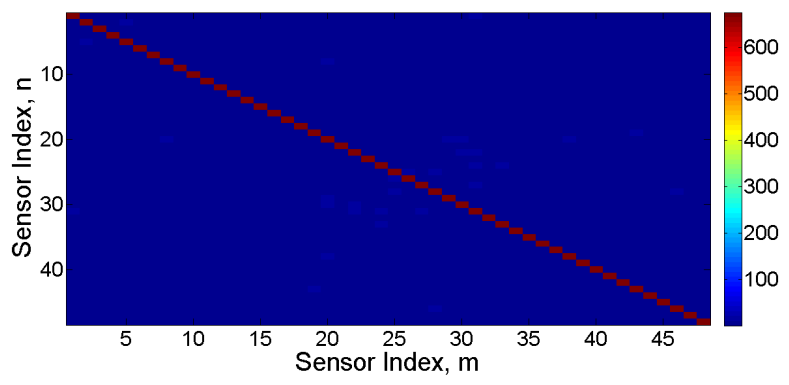

(b) Random array

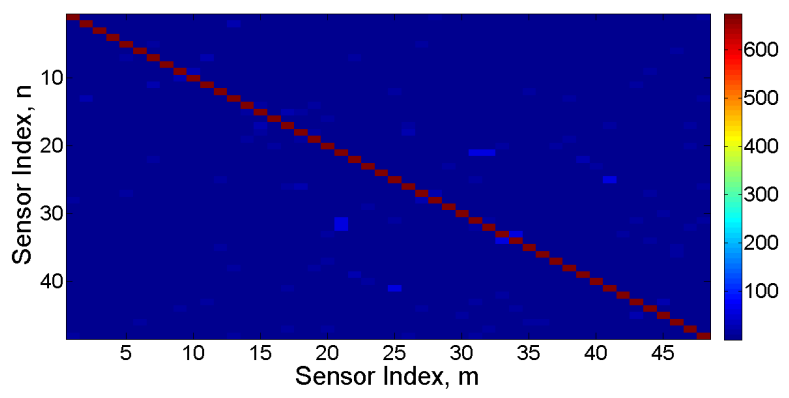

(c) Danzer array

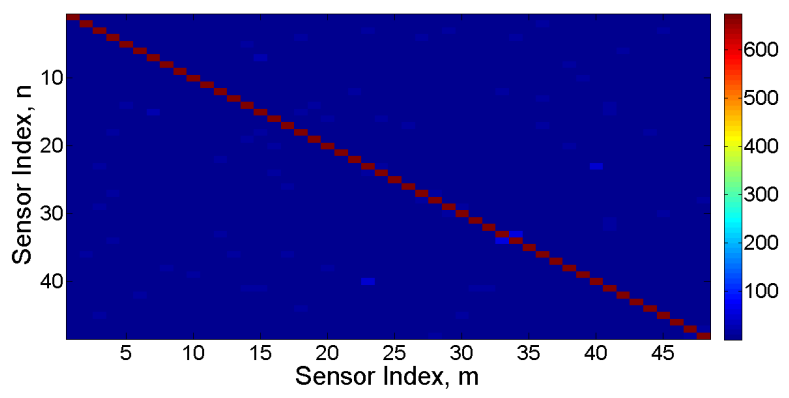

(d) Optimized Danzer array

Figure 5.11: Correlation matrices for different geometries using Danzer tile as aperture. High energy in off-diagonal elements indicate high mutual coherence. 


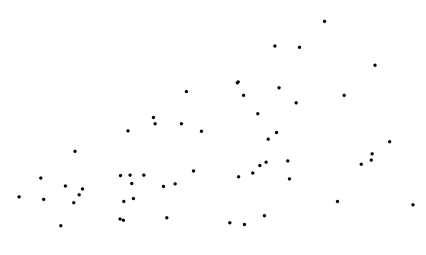

(a)

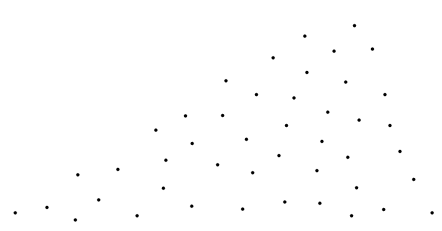

(b)

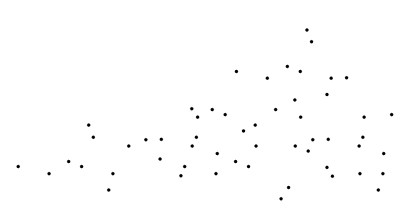

(c)

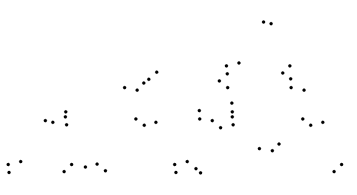

(d)

Figure 5.12: a) 55-element random array, b) 55-element constrained random array, d) 55-element Penrose array, e) 55-element optimized Penrose array. 
geometry studied earlier in this section, which had 48 elements. Using the procedure summarized in Algorithm 6, we find the optimum points and generate the geometry through the process of inflation, as shown in Figure 5.12d. Figure 5.12a-c shows other geometries that have been studies for performance comparison. The geometry in Figure 5.12a has been generated through a random element generator that would place 55 random elements in the aperture specified by Penrose triangle $A L$. Similarly, the geometry shown in Figure $5.12 \mathrm{~b}$ has been generated to a random element generator but with a constraint the no two elements lie within $\lambda / 2$ of each other. The motivation behind such a constraint is to restrain the element from collapsing into each other, which happens in case of unconstrained random geometry. Figure 5.12c shows the Penrose geometry generated by choosing the initial point $\boldsymbol{p}=[0.5,0.5]$.

Figure 5.13 shows the correlation of the four geometries discussed earlier. The ideal form of this matrix is close to identity matrix. Any matrix with high offdiagonal elements has high mutual-coherence between its rows and will perform poorly in CS scenario.

One aspect of the array geometry is the distance between neighboring elements in the array. We define three parameters $d_{\min }, d_{\max }$ and $d_{a v}$ to gage different aspects of the geometries being studied. The parameters are given by

$$
\begin{aligned}
d_{\text {min }} & =\min \left(d_{n}\right), \\
n & =1,2, \ldots, N \\
d_{\text {max }} & =\max \left(d_{n}\right), \\
n & =1,2, \ldots, N \\
d_{\text {avg }} & =\frac{1}{N} \sum_{n=1}^{N} d_{n} .
\end{aligned}
$$




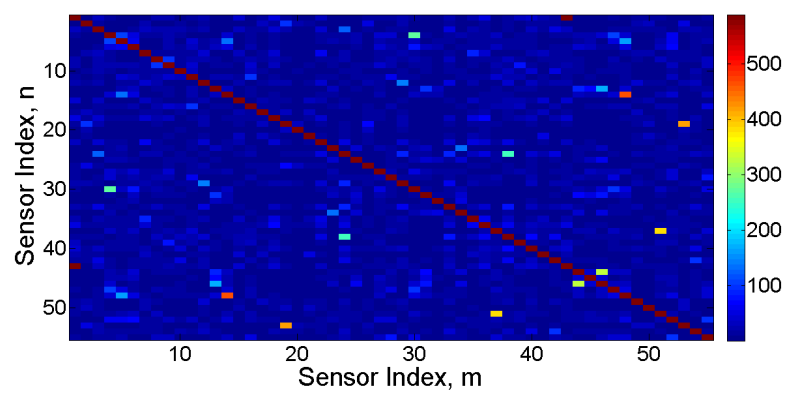

(a) Random array

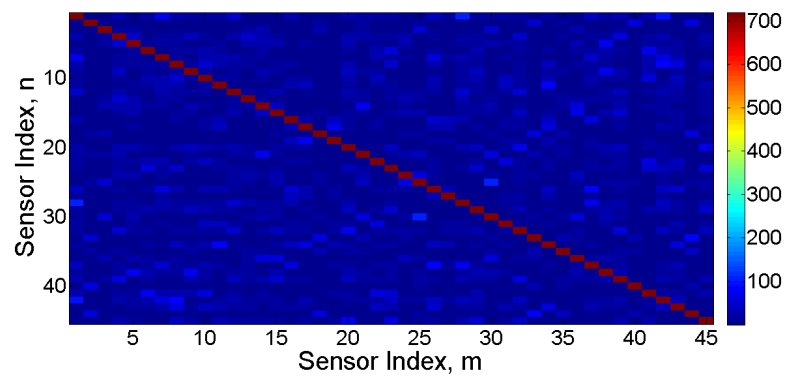

(b) Constrained random array

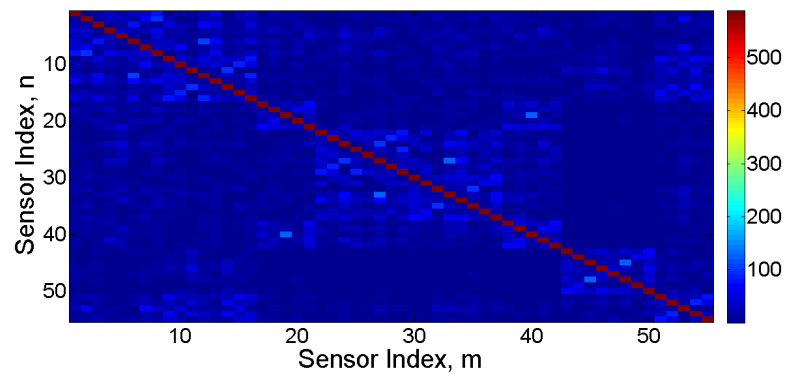

(c) Penrose array

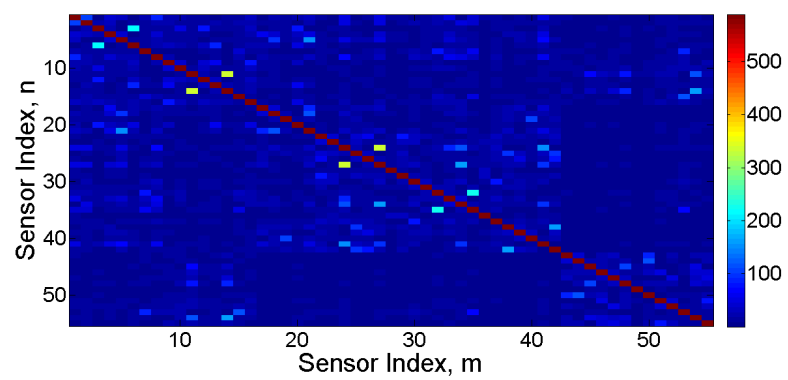

(d) Optimized Penrose array

Figure 5.13: Correlation matrices for different geometries using Penrose tile as aperture. High energy in off-diagonal elements indicate high mutual coherence. 
Table 5.1 and Table 5.2 show these parameters for different types of geometries used in this study. There are three parameters, the minimum nearest neighbor distance, $d_{\text {min }}$, the maximum nearest neighbor distance, $d_{\text {max }}$ and the average nearest neighbor distance, $d_{a v}$. In the table, these values are normalized through dividing each value by $\lambda$, the wavelength of the center frequency. For Danzer geometries shown in Table 5.1, rectangular-grid array has all the values 0.5 since the separation between nearest neighbors is fixed to $\lambda / 2$. For random array without any constraints, $d_{\min }$ is lowest as compared to all other configurations while $d_{\max }$ is highest as compared to the rest of the configurations. $d_{a v}$, the average nearest neighbor distance is comparable to rectangular grid array. This shows that the utilization of aperture in this configuration is not optimal as some of the array elements are too close and some are too much far apart. The values of parameters for random array configuration, but with a constraint of $\lambda / 3$ on minimum nearest neighbor separation, are somewhat reasonable as compared to completely random configuration. $d_{\text {min }}$ for this array is slightly greater than $\lambda / 3$ which is quite expected. The values for maximum and average separation, $d_{\max }$ and $d_{a v}$, are also reasonable. For Danzer array configuration, the value of $d_{\min } / \lambda$ is 0.14 , definitely better than completely random configuration but still less than desirable. For optimal configuration, the minimum separation, $d_{\text {min }}$, is quite close to $\lambda / 3$ and maximum separation, $d_{\max }$ is close to $1.27 \lambda$. The average separation, $d_{\text {avg }}$ for optimal configuration is the greatest as compared to the rest of configurations.

For Penrose geometries listed in Table 5.2 we can see that some elements almost collapsed which is indicated by the value of 0.06 for $d_{\min } / \lambda$. For constrained random geometry the minimum distance is greater than $\lambda / 3$, while the elements are evenly spread within the aperture as indicated by $d_{\text {avg }}$. The optimized-Penrose geometry has greater average inter-element distance as compared to the Penrose geometry. 
Table 5.1: Geometrical properties of different antenna array designs using Danzer array geometry (Figure 5.10). The minimum nearest neighbor distance(5.75). The average nearest neighbor distance(5.77). The maximum nearest neighbor distance(5.76)

\begin{tabular}{|l|c|c|c|c|}
\hline Array Configuration & $\begin{array}{c}\text { No. of } \\
\text { elements }\end{array}$ & $\frac{d_{\min }}{\lambda}$ & $\frac{d_{a v}}{\lambda}$ & $\frac{d_{\max }}{\lambda}$ \\
\hline Random & 48 & 0.0918 & 0.52 & 1.62 \\
\hline Random - constrained & 48 & 0.36 & 0.59 & 1.03 \\
\hline Danzer & 48 & 0.14 & 0.60 & 1.47 \\
\hline Danzer - optimized & 48 & 0.316 & 0.69 & 1.27 \\
\hline Rectangular & 179 & 0.5 & 0.5 & 0.5 \\
\hline
\end{tabular}

Looking at Figures 5.12c and d, we can see that the array elements in the optimum Penrose geometry exhibit some clustering behavior, but interestingly enough the elements are not collapsing as the minimum inter-element distance is still larger than the Penrose array.

Following we compare the performance of each of these array configurations in regards to estimating the direction of arrival of three closely located sources. We use these array geometries to estimate three target directions, located at 19, 20 and 21 degrees azimuth with amplitudes 1.5, 1.0 and 1.5 respectively. All the sources are located at the same elevation of $25^{\circ}\left(\psi=25^{\circ}\right)$.

Figure 5.14 shows the mean squared error (MSE) plot for all five geometries 
Table 5.2: Geometrical properties of different antenna array designs using Penrose array geometry (Figure 5.12). The minimum nearest neighbor distance (5.75). The average nearest neighbor distance (5.77). The maximum nearest neighbor distance $(5.76)$

\begin{tabular}{|c|c|c|c|c|}
\hline Array Configuration & $\begin{array}{l}\text { No. of } \\
\text { elements }\end{array}$ & $\frac{d_{\min }}{\lambda}$ & $\frac{d_{a v}}{\lambda}$ & $\frac{d_{\max }}{\lambda}$ \\
\hline Random & 55 & 0.06 & 0.29 & 0.75 \\
\hline Random - constrained & 55 & 0.47 & 0.66 & 1.02 \\
\hline Penrose & 55 & 0.38 & 0.55 & 0.89 \\
\hline Penrose - optimized & 55 & 0.46 & 0.60 & 0.88 \\
\hline
\end{tabular}

shown in Figure 5.10. MSE has been calculated using the following equation:

$$
M S E=\frac{1}{N} \sum_{n=1}^{N}\left(x_{n}-\hat{x}_{n}\right)^{2} .
$$

$\hat{\boldsymbol{x}}$ is the estimate of $\boldsymbol{x}$, which is the output of the following $l_{1}$ minimization program:

$$
\begin{aligned}
\underset{x}{\operatorname{minimize}} & \|x\|_{1} \\
\text { subject to } y & =A x .
\end{aligned}
$$

We can see in Figure 5.14 that the triangular array (with rectangular grid sampling, Figure 5.10c) has the largest MSE, followed by Danzer array (Figure 5.10d), although the number of elements (179) in the rectangular grid array is far greater than the number of elements (48) in the Danzer array. Constrained random array 


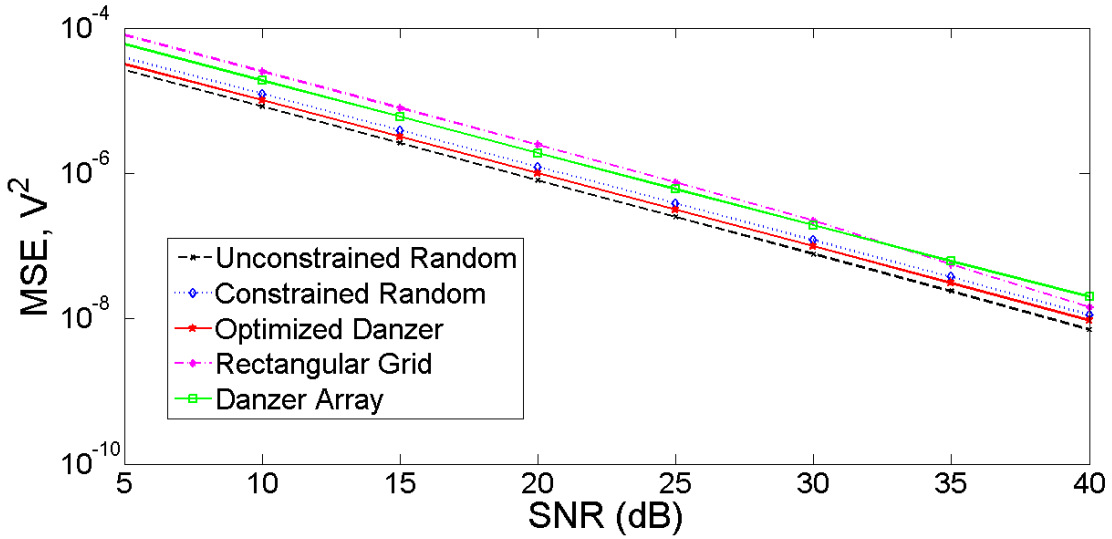

Figure 5.14: Mean Squared Error for different SNR values plotted for different geometries shown in Figure 5.10 based on Danzer tile-II aperture.

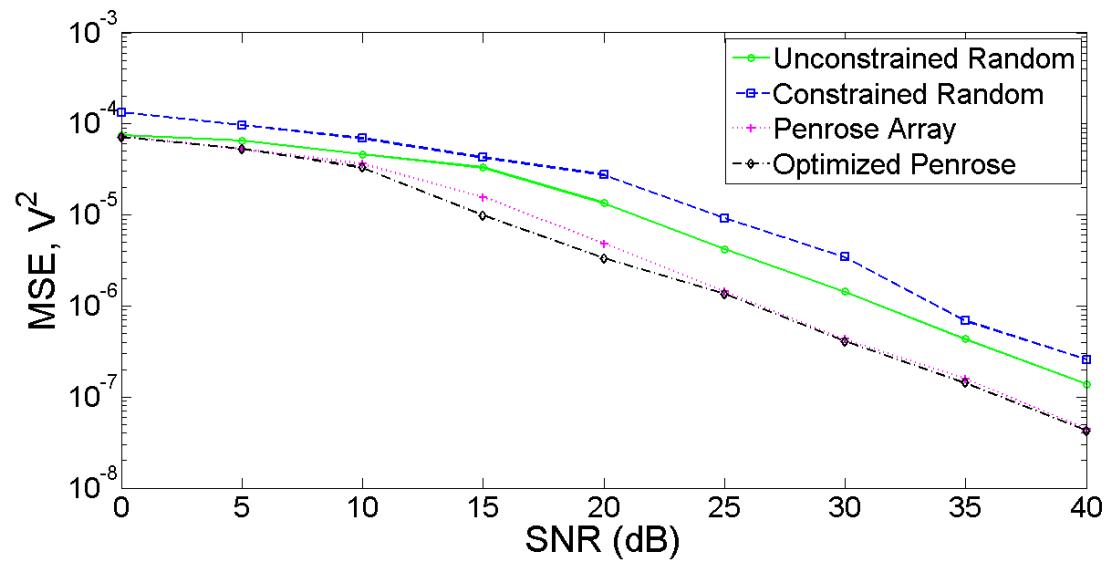

Figure 5.15: Mean Squared Error for different SNR values plotted for different geometries shown in Figure 5.12 based on Penrose tile- $A L$ aperture 
(Figure 5.10b) performed better than the Danzer array. Random array configuration (Figure 5.10a) has slightly better performance than optimized aperiodic array configuration (Figure 5.10e).

Figure 5.15 shows a plot for MSE with respect to SNR for different geometries shown in Figure 5.12. The performance of Penrose and optimized Penrose geometries is significantly better than the two iterations of the random geometries shown in Figure 5.10a and Figure 5.10b.

\subsection{Conclusion}

In this Chapter, we introduced a design technique that is capable of being used for generation of antenna-sensors layouts based on aperiodic tilings, specifically Danzer tilings in the context of compressive sensing. This technique generates antennasensor layouts through a slight disturbance of the inflation process through which aperiodic geometries can be generated. We have devised an optimization scheme that is generic in nature and can be applied to other aperiodic geometries whether based on inflation process or some other geometrical transformations (translations, scaling, rotations, etc.) of base geometries.

Usually, aperiodic arrays are generated through either turning off array elements or disturbing their positions on a large grid of array elements. Such schemes require optimization of a large number of parameters. Our scheme requires a very few parameters that needs optimization. This scheme can be utilized in arrays that consist of several sub-arrays. Due to the inherent aperiodic structure such arrays are inherently sparse and well suited for applications that are using powerful capabilities of compressive sensing algorithms that exploits sparsity in some domain (in our case 
CHAPTER 5. ARRAY GEOMETRY DESIGN FOR NARROW BAND COMPRESSIVE SENSING DOA ESTIMATION

it is source-space). 


\section{Chapter 6}

\section{Array Geometry Design for}

\section{Broadband Compressive Sensing}

\section{DOA Estimation}

\subsection{Introduction}

In Chapter 4, we discussed the performance of different broadband algorithms developed specifically for direction of arrival (DOA) estimation problem in compressive sensing scenario. We developed a number of algorithms to solve this problem and evaluated their performance.

In this Chapter, we will focus on the geometry aspect of the compressive sensing algorithm for the broadband case. We have seen in Chapter 5 that the geometry of the sensor array plays a key role in the performance of the CS algorithm. In that chapter, we used aperiodic geometry to address the problem of orthogonality of the 
basis matrix. For $l_{1}$ optimization problem, the mutual coherence results have been discussed in section 5.2. The $l_{1}$ minimization algorithm for broadband case does not perform very well. The alternative is to use a greedy algorithm like SOMP, used in Chapter 4). We used Uniform Linear Array (ULA) geometry in that case. Here, we study different kind of geometries for the broadband SOMP algorithm studied in Chapter 4.

\subsection{Geometry Optimization Problem for Broad- band DOA Estimation}

For the broadband case, the measurement matrix depends on three parameters, $\theta, r$ and $f$. $\theta$ is the steering direction, $r$ is the position of an array element and $f$ is the frequency of the signal. Note that in the narrowband case, the parameter $f$ is absent as we have only a very narrow band of frequencies which can be approximated as a single frequency. Here, the measurement matrix for the broadband case, $\boldsymbol{A}(r, \theta, f)$, can be discretized and written in the following manner:

$$
\boldsymbol{A}(l, m, n)=e^{j \kappa_{l} \boldsymbol{p}_{m}^{T} \boldsymbol{r}_{n}}, l=\{1, \ldots, L\}, m=\{1, \ldots, M\}, n=\{1, \ldots, N\},
$$

Here, $\kappa_{l}$ is the magnitude of wavenumber vector for frequency bin $l$, given by ex-

pression $k_{l}=\frac{2 \pi f_{l}}{c}, r_{n}$ is the normalized direction vector for direction $n$ and $p_{m}$ is the position vector for sensor $m$. $L$ is the number of frequency bins, $M$ is the number of array sensors, while $N$ is the number of directions. 2-D arrays have the capability of resolving targets both in azimuth and elevation, so the number $\mathrm{N}$ represents all the directions in elevation and azimuth.

We define a matrix, $\boldsymbol{B}=\boldsymbol{A} \boldsymbol{A}^{H}$. The matrix $\boldsymbol{B}$ gives the dot product of the 
rows of the matrix $\boldsymbol{A}$ and for frequency index $l$ and it is given by:

$$
\boldsymbol{B}_{l}(u, v)=\sum_{\xi=1}^{N} e^{j \kappa_{l}\left(\boldsymbol{p}_{u}^{T} \boldsymbol{r}_{\xi}-\boldsymbol{p}_{v}^{T} \boldsymbol{r}_{\xi}\right)} .
$$

Note that we have $L$ such matrices, one matrix each for every value of frequency index $l$. In narrowband case we just had one matrix $\boldsymbol{B}$.

We have seen in Section 5.6.6, that the aperiodic array is manufactured by starting with just three points. Thus each element of the array $\boldsymbol{p}_{u}$, can be written as $\boldsymbol{T}_{u} \boldsymbol{p}$, where, $\boldsymbol{p}$ contains the initial two or three points, depending on the base geometry. Making this transformation, we get

$$
\boldsymbol{B}_{l}(u, v)=\sum_{\xi=1}^{N} e^{j \kappa_{l}\left(\left(\boldsymbol{T}_{u} \boldsymbol{p}\right)^{T} \boldsymbol{r}_{\xi}-\left(\boldsymbol{T}_{v} \boldsymbol{p}\right)^{T} \boldsymbol{r}_{\xi}\right)} .
$$

Discarding the summation notation, and expanding the expression, we get

$$
\boldsymbol{B}_{l}(u, v)=e^{j \kappa_{l}\left(\left(\boldsymbol{T}_{u} \boldsymbol{p}\right)^{T} \boldsymbol{r}_{1}-\left(\boldsymbol{T}_{v} \boldsymbol{p}\right)^{T} \boldsymbol{r}_{1}\right)}+\ldots+e^{j \kappa_{l}\left(\left(\boldsymbol{T}_{u} \boldsymbol{p}\right)^{T} \boldsymbol{r}_{N}-\left(\boldsymbol{T}_{v} \boldsymbol{p}\right)^{T} \boldsymbol{r}_{N}\right)}
$$

Since $\left[\left(\boldsymbol{T}_{u} \boldsymbol{p}\right)^{T}-\left(\boldsymbol{T}_{v} \boldsymbol{p}\right)^{T}\right] \boldsymbol{r}_{\xi}$ are scalars, we can transpose them to get the following expression:

$$
\begin{aligned}
{\left[\left(\boldsymbol{T}_{u} \boldsymbol{p}\right)^{T}-\left(\boldsymbol{T}_{v} \boldsymbol{p}\right)^{T}\right] \boldsymbol{r}_{\xi} } & =\boldsymbol{r}_{\xi}^{T}\left[\left(\boldsymbol{T}_{u} \boldsymbol{p}\right)^{T}-\left(\boldsymbol{T}_{v} \boldsymbol{p}\right)^{T}\right]^{T} \\
& =\boldsymbol{r}_{\xi}^{T}\left[\boldsymbol{T}_{u} \boldsymbol{p}-\boldsymbol{T}_{v} \boldsymbol{p}\right] \\
& =\boldsymbol{r}_{\xi}^{T}\left[\boldsymbol{T}_{u}-\boldsymbol{T}_{v}\right] \boldsymbol{p}
\end{aligned}
$$

When $u=v$, the argument of exponentials all go to zero, and we have

$$
\sum_{\xi=1}^{N} e^{j \kappa_{0} 0}=\mu .
$$

This condition can be satisfied if we multiply each column of $\boldsymbol{A}$ by $\frac{\mu}{N}$.

For diagonal entries, the orthogonality condition is satisfied if,

$$
e^{j \kappa_{l}\left(\boldsymbol{r}_{1}^{T}\left[\boldsymbol{T}_{u}-\boldsymbol{T}_{v}\right] \boldsymbol{p}\right)}+\ldots+e^{j \kappa_{l}\left(\boldsymbol{r}_{N}^{T}\left[\boldsymbol{T}_{u}-\boldsymbol{T}_{v}\right] \boldsymbol{p}\right)}=0 .
$$


We can choose the arguments of exponentials (on a unit circle) in such a way that their sum becomes equal to zero, that is,

$$
e^{j \epsilon_{1}^{l}}+e^{j \epsilon_{2}^{l}}+\ldots+e^{j \epsilon_{N}^{l}}=0 .
$$

This gives rise to the following set of equations:

$$
\begin{gathered}
\boldsymbol{r}_{1}^{T}\left[\boldsymbol{T}_{u}-\boldsymbol{T}_{v}\right] \boldsymbol{p}=\epsilon_{1} \\
\cdot \\
\boldsymbol{r}_{N}^{T}\left[\boldsymbol{T}_{u}-\boldsymbol{T}_{v}\right] \boldsymbol{p}=\epsilon_{N}
\end{gathered}
$$

Here, $\epsilon_{i}=\frac{\epsilon_{i}^{l}}{k^{l}}$.

All the combined equations for indices $u, u \in\{1, . . M\}, v, v \in\{1, \ldots, M\}$ and $l, l \in\{1, . ., L\}$ could be written into one huge matrix equation,

$$
\Upsilon p=E .
$$

Here,

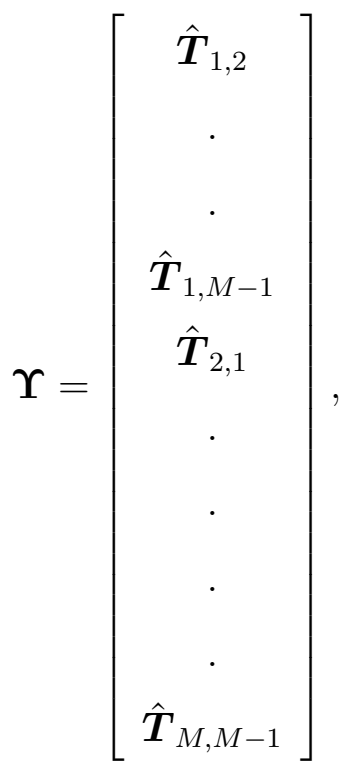




$$
\boldsymbol{E}=\left[\boldsymbol{\epsilon}^{T}, \boldsymbol{\epsilon}^{T} \ldots \boldsymbol{\epsilon}^{T}\right]^{T}
$$

The optimization problem now becomes:

$$
\begin{array}{ll}
\underset{\boldsymbol{p}}{\operatorname{minimize}} & \|\boldsymbol{\Upsilon} \boldsymbol{p}-\boldsymbol{E}\|_{2} \\
\text { subject to } & \boldsymbol{M}_{u} \boldsymbol{p}+\boldsymbol{C}_{u} \leq 0 .
\end{array}
$$

This is a least squares problem with linear inequality constraints and is a convex problem, if the constraints are well defined.

\subsection{Boundary Constraints for Optimization Prob- lem}

$$
\boldsymbol{M}_{u} \boldsymbol{p}_{u}+\boldsymbol{C}_{u} \leq 0
$$

The boundary constraints (6.16) have already been discussed in detail in Section 5.8. The boundary constraints for (6.15) are shown in Figure 6.1 for Danzer geometry and Figure 6.2 for Penrose geometry. 


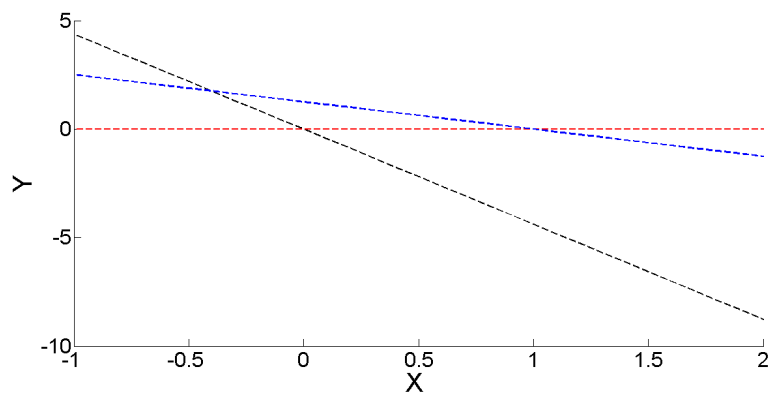

(a)

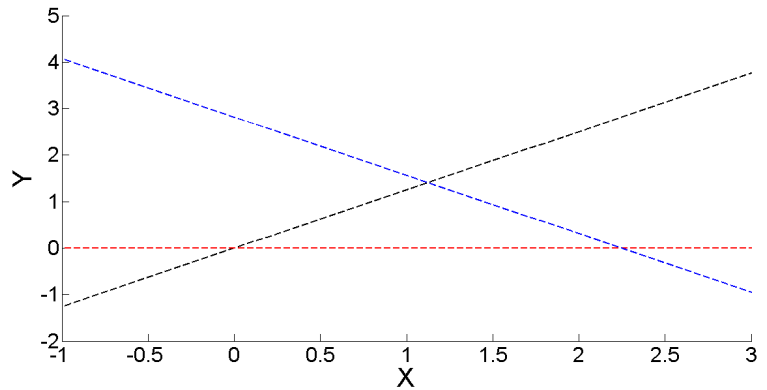

(b)

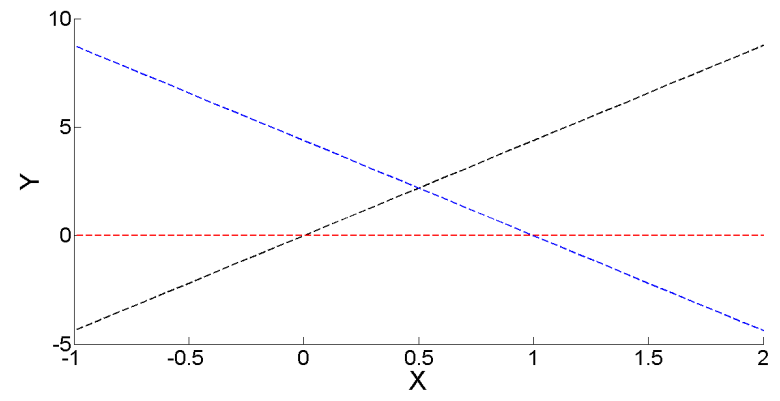

(c)

Figure 6.1: Optimization constraints depicted by lines for a) Danzer Tile 1, b) Danzer Tile 2, c) Danzer Tile 3. 


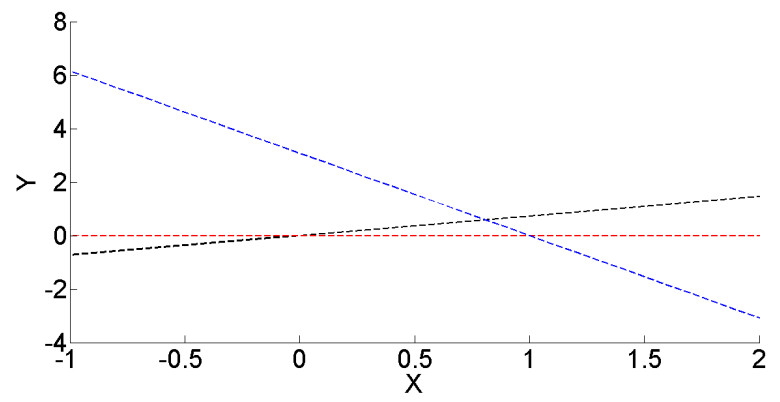

(a)

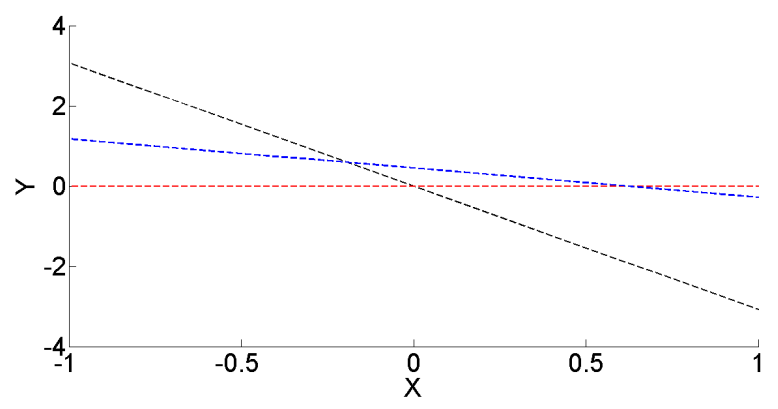

(b)

Figure 6.2: Optimization constraints depicted by lines for a) Penrose Tile AL, b) Penrose Tile AS. 
CHAPTER 6. ARRAY GEOMETRY DESIGN FOR BROADBAND COMPRESSIVE SENSING DOA ESTIMATION

\subsection{Optimization Results for Aperiodic Geome- tries}

\subsubsection{Simulation Setup}

The constrained least squares optimization (6.15) was performed for the following set of geometries:

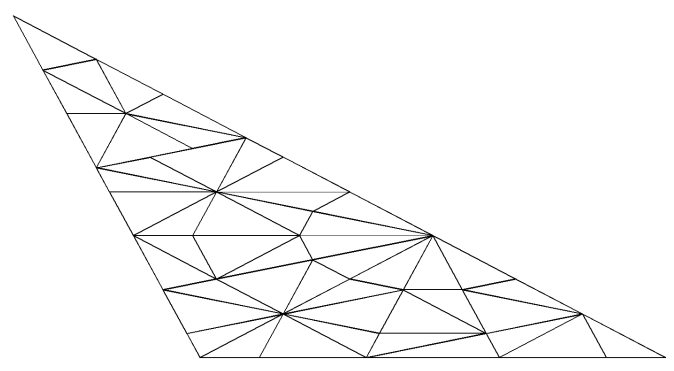

Figure 6.3: Level IV Penrose Tile AL (Danzer Geometry 1).

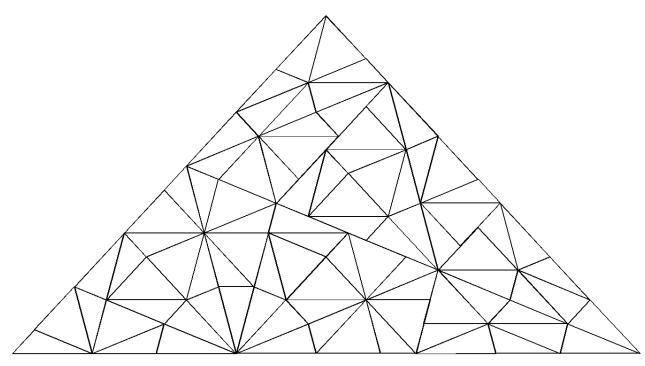

Figure 6.4: Level II Danzer Tile 1 (Danzer Geometry 2). 


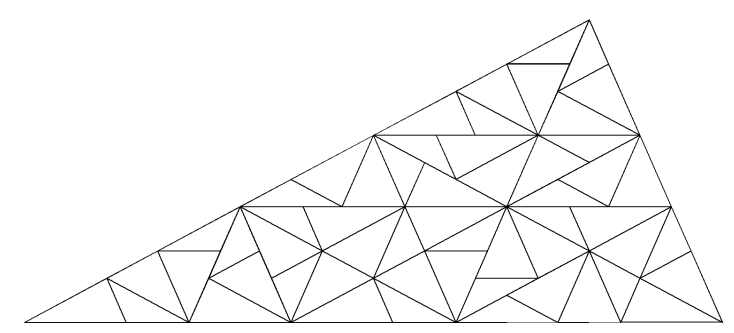

Figure 6.5: Level II Danzer Tile 2 (Penrose Geometry 1).

i Tile-1 Danzer Geometry at second iteration of inflation process, shown in Fig 6.3 (Henceforth refered to as "Danzer Geometry 1"). For all kinds of aperture sampling (including rectangular, random, etc.), the aperture for this geometry would always be the same, i.e., Tile-1 of Danzer tiling.

ii Tile-2 Danzer Geometry at second iteration of inflation process, shown in Fig 6.4 (Henceforth refered to as "Danzer Geometry 2"). For all kinds of aperture sampling (including rectangular, random, etc.), the aperture for this geometry would always be the same, i.e., Tile-2 of Danzer tiling.

iii Tile-AL Penrose Geometry at fourth iteration of inflation process, shown in Fig 6.5 (Henceforth refered to as "Penrose Geometry 1"). For all kinds of aperture sampling (including rectangular, random, etc.), the aperture for this geometry would always be the same, i.e., Tile-AL of Danzer tiling.

The reason for choosing such an unconventional aperture is that our study relates to aperture sampling rather than the antenna aperture itself. Although antenna aperture does have some bearing on the output of the algorithm but we are more interested in the sampling of the aperture in this study. For this reason we have 
kept things simple by choosing the aperture as the base triangle for the respective geometry.

Table 6.1: Parameters used for the optimization problem in (6.15)

\begin{tabular}{|l|c|c|c|}
\hline Parameter & Danzer Geometry 1 & Danzer Geometry 2 & Penrose Geometry 1 \\
\hline Number of sensors & 48 & 87 & 45 \\
\hline Sampling frequency $\left(f_{s}\right)$ & 1 & 1 & 1 \\
\hline Centre frequency $\left(f_{c}\right)$ & 0.3 & 0.3 & 0.3 \\
\hline Bandwidth & 0.24 & 0.24 & 0.24 \\
\hline
\end{tabular}

After solving the optimization problem (6.15) for all three geometries, we get three points for Danzer Geometry 1 as shown in Fig 6.6. All the three points lie on the boundary of the triangles. The result of optimization problem (6.15) is shown in Figure 6.7. In this case the optimized points are close to the boundary but not located on the boundary itself. The result of optimization problem for Penrose Geometry 1 is shown in Figure 6.8. Here one point is located on the boundary (Tile $\mathrm{AL})$ but the other point is away from the boundary (Tile AS). 


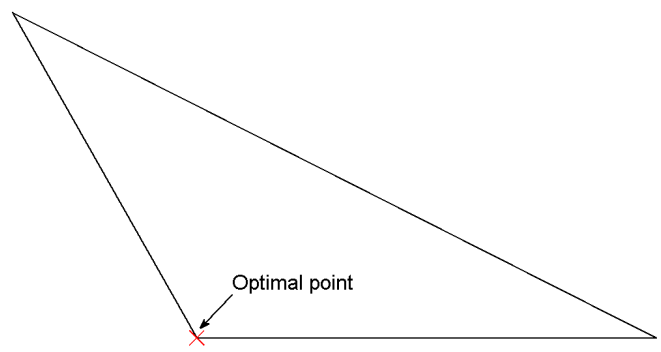

(a)

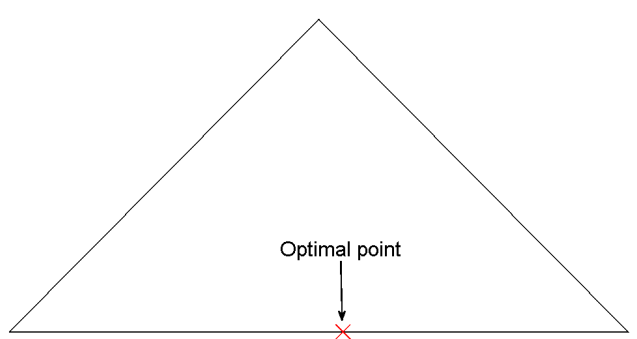

(b)

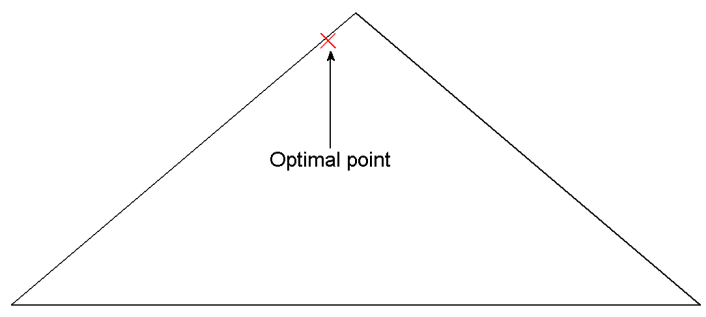

(c)

Figure 6.6: Optimal points for Danzer Geometry 1 a) Danzer Tile 1, b) Danzer Tile 2, c) Danzer Tile 3. 


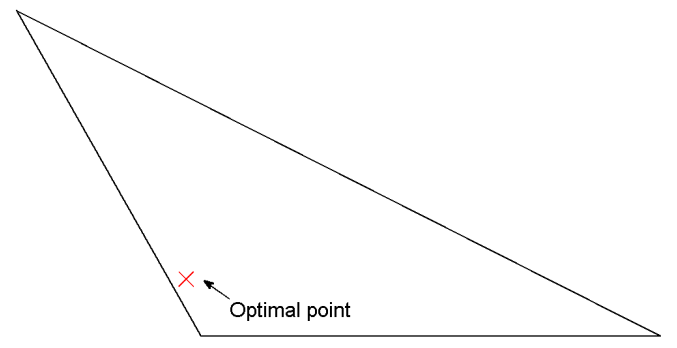

(a)

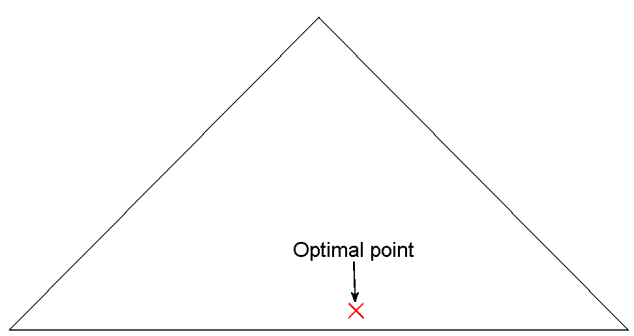

(b)

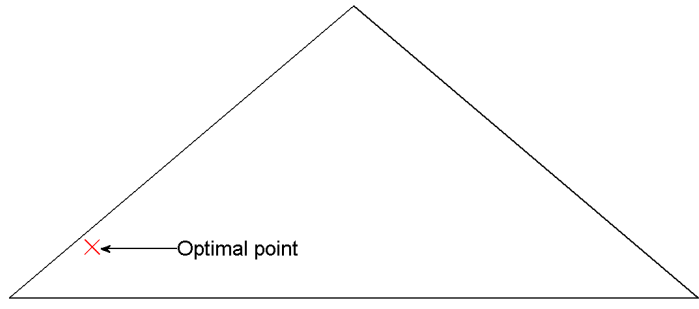

(c)

Figure 6.7: Optimal points for Danzer Geometry 2 geometry a) Danzer Tile 1, b) Danzer Tile 2, c) Danzer Tile 3. 


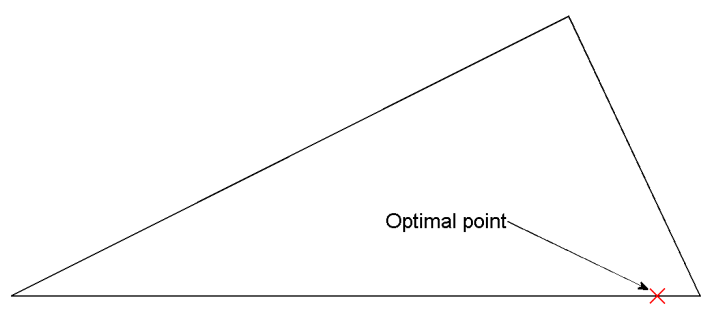

(a)

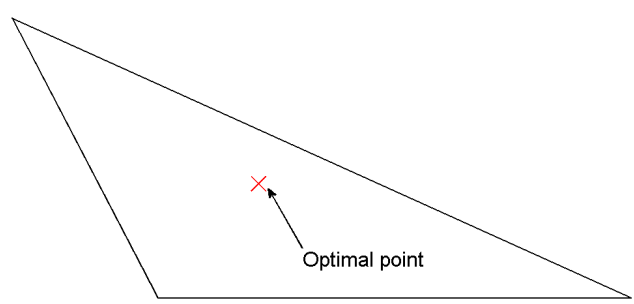

(b)

Figure 6.8: Optimal points for Penrose Geometry 1 a) Penrose Tile AL, b) Penrose Tile AS. 


\subsubsection{Resultant Geometries}

After finding the optimized points, we can form the optimum sensor configuration for each case. To evaluate the performance of the optimized geometries, we need other configurations for comparison. We have used the following configurations:

i Random: Within the triangular aperture, we generate random samples using a uniform distribution. In Chapter 5, we have seen that the random sampling is very useful as it produces a measurement matrix having near orthogonal columns.

ii Constrained Random: Since random geometry is not practical, as it would almost always contain samples too close to each other, we constrain the minimum spacing between two neighboring elements to $\lambda / 3$. This way the pseudorandomness of the sampling is preserved but having the advantage of being practically implementable.

iii Uniformly Sampled: This form of sampling is the 2-D equivalent of Uniform Linear Array (ULA) but with the aperture constrained to a triangle instead of a square or a rectangle.

iv Base Geometry: This sampling is generated by taking arbitrary point, $(0.5,0.5)$, inside each base triangle and then generating the complete geometry using the transformation matrix from Section 5.6.5.

v Optimized: We take the optimized points and generate the complete geometry using the transformation matrix derived in Section 5.6.5. 


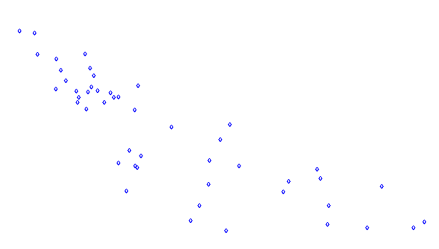

(a)

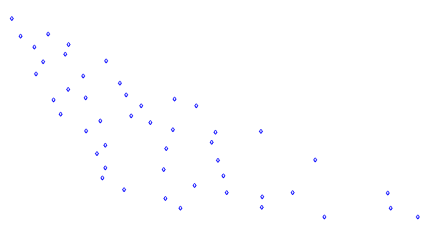

(b)

(c)

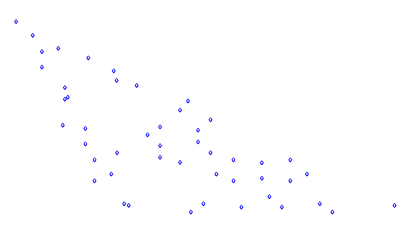

(d)

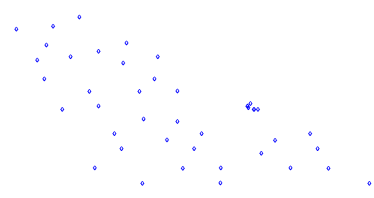

(e)

Figure 6.9: Danzer Tile 1 aperture a) 48-element random array, b) 48-element constrained random array, c) 60-element triangular array (rectangular grid sampling), d) 48-element Danzer array, e) 48-element optimized aperiodic array. 
Figure 6.9 shows different geometries generated using the "Danzer Geometry 1" aperture. The aperture for each geometry is the same (i.e Danzer Tile 1 (Figure 6.3)) for all the array configurations. The Horizontal distance between elements in uniformly sampled configuration is $d=\frac{\lambda_{\min }}{2}$. Where $\lambda_{\min }=\frac{c}{f_{\max }}$ and $f_{\max }=f_{c}+$ $B W / 2$. $f_{c}$ being the center frequency and $B W$ is the bandwidth of the broadband signal. As a result of optimization, the element spacing for the optimal geometry has reduced as compared to Danzer array (Fig 6.9d).

Figure 6.10 shows different geometries generated using the Danzer Geometry 2 aperture. The aperture for each geometry is the same i.e Danzer Tile 2 (Figure 6.4). The Horizontal distance between elements in uniformly sampled configuration is $d=\frac{\lambda_{\min }}{2}$.

Figure 6.11 shows different geometries generated using the Penrose Geometry 1 aperture. The aperture for each geometry is the same, i.e, Penrose Tile AL (Figure 6.5). The Horizontal distance between elements in uniformly sampled configuration is $d=\frac{\lambda_{\min }}{2}$.

\subsection{Performance Results}

In this section, we analyze the performance of different geometries that we have studied till now. We will analyze the performance of the algorithms at different SNR values, ranging from really poor $(-35 \mathrm{db})$ to good SNR $(25 \mathrm{db})$. Since we are 


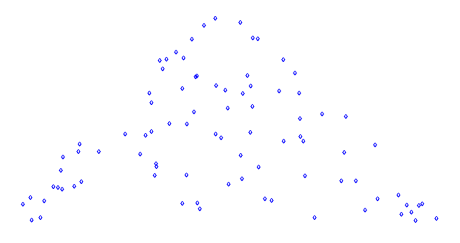

(a)

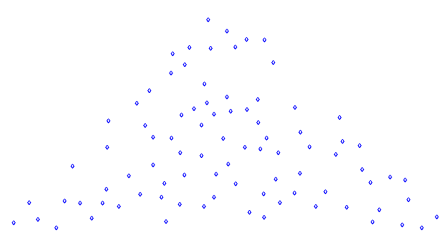

(b)

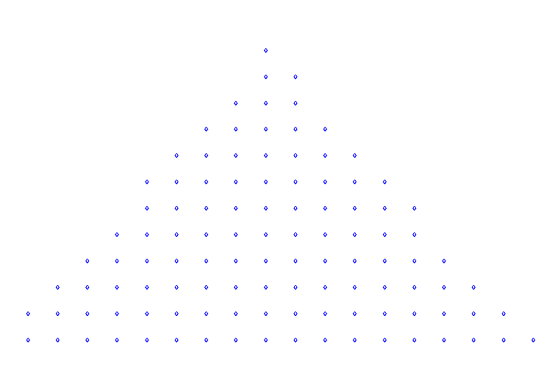

(c)

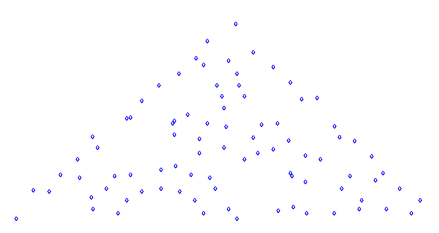

(d)

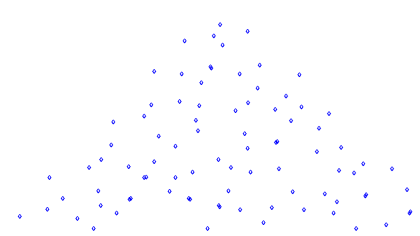

(e)

Figure 6.10: Danzer Tile 2 aperture a) 87-element random array, b) 87-element constrained random array, c) 111-element triangular array (rectangular grid sampling), d) 87-element Danzer array, e) 87-element optimized aperiodic array. 


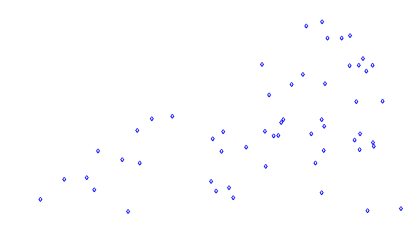

(a)

(b)

(c)

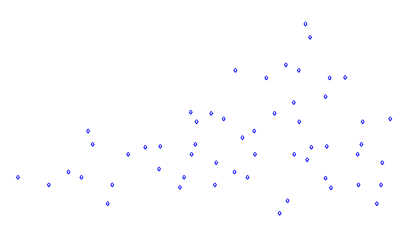

(d)

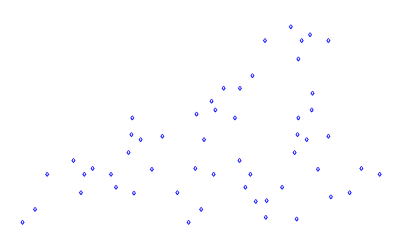

(e)

Figure 6.11: Penrose Tile AL aperture a) 45-element random array, b) 45-element constrained random array, c) 48-element uniform sampling array, d) 45-element Danzer array, e) 45-element optimized aperiodic array. 
Table 6.2: Parameters for simulation

\begin{tabular}{|l|c|c|c|}
\hline Parameter & Danzer Geometry 1 & Danzer Geometry 2 & Penrose Geometry 1 \\
\hline Number of sensors & 48 & 87 & 45 \\
\hline Sampling frequency $\left(f_{s}\right)$ & 1 & 1 & 1 \\
\hline Centre frequency $\left(f_{c}\right)$ & 0.3 & 0.3 & 0.3 \\
\hline Bandwidth & 0.22 & $20 \lambda_{\min }$ & 0.22 \\
\hline Horizontal aperture & $12 \lambda_{\min }$ & $78,94,114$ & $20 \lambda_{\min }$ \\
\hline Targets Azimuth $($ degrees $)$ & $60,94,144$ & 0 & $78,94,114$ \\
\hline Targets Elevation $($ degrees $)$ & 0 & 256 & 256 \\
\hline Frequency bins & 256 & 2 & 2 \\
\hline Snapshots & 2 & 3 & 3 \\
\hline Detection window (degrees) & 4 & 0.3 & 0 \\
\hline
\end{tabular}


dealing with compressive sensing scenario, the time domain sanpshots are kept at the minimum $(r=2)$.

Table 6.2 lists the parameters used for 200 Montecarlo simulations that were performed to evaluate the performance of the SOMP broadband algorithm in Section (4.4) for all the geometries discussed in Section (6.4.2). The reason for choosing SOMP algorithm as compared to others discussed in Chapter 4 was that the algorithm does not assume a uniform linear sampling, as is the case with focusing based approaches, discussed in that chapter. We define a window around the actual angle of arrival, $\theta$, if the estimated angle $\hat{\theta}$ lies within that window, it is considered detected. For more that one target (as in our case, we have three targets), for each iteration of the Montecarlo simulation, if all the targets lie within the detection window, only then we considered the detection successful, otherwise it is considered a failure. This definition is an extension of the method described in [37]. In [37], the author has discussed the resolution of two targets, while in our case we are using the definition for detection/resolution of more than two targets. Our criterion is stricter than the one developed in [37].

The mean squared error for each target is evaluated over $\mathrm{N}$ simulations using the following equation:

$$
M S E=\frac{1}{N} \sum_{i=1}^{N}(\theta-\hat{\theta})^{2} .
$$

In the case of "Danzer Geometry 1", Figure 6.12 shows the performance of the five different array configurations discussed in Section 6.4.2. The performance of the SOMP algorithm for the optimized geometry is much better as compared to the other geometries for the simulation parameters given in Table 6.2. Provided that the uniform sampling geometry has significantly more elements than the other configurations, its performance is still the worse. 


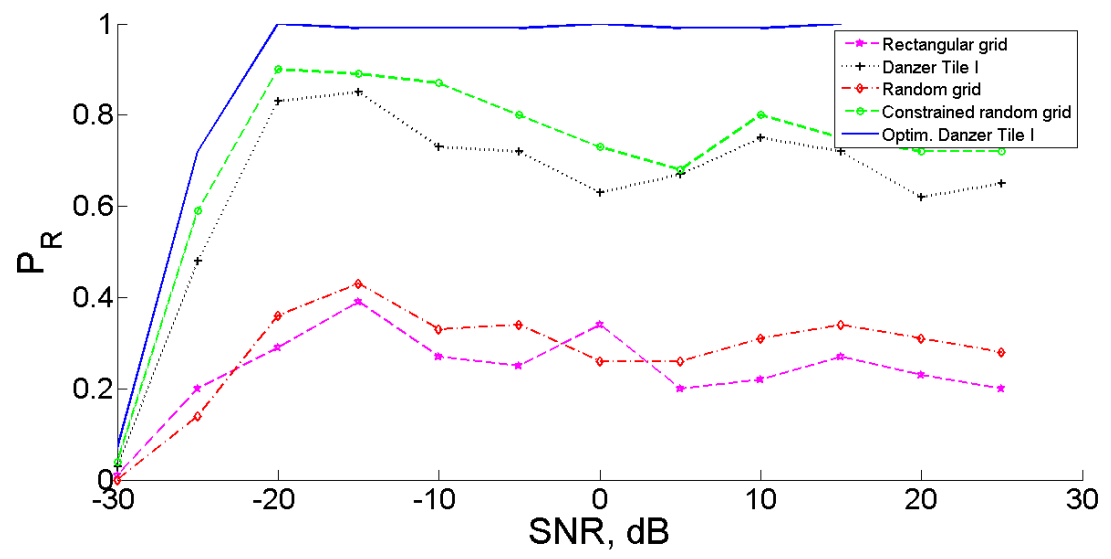

Figure 6.12: SNR vs the probability of resolution $\left(P_{R}\right)$ for Danzer Tile 1 aperture.

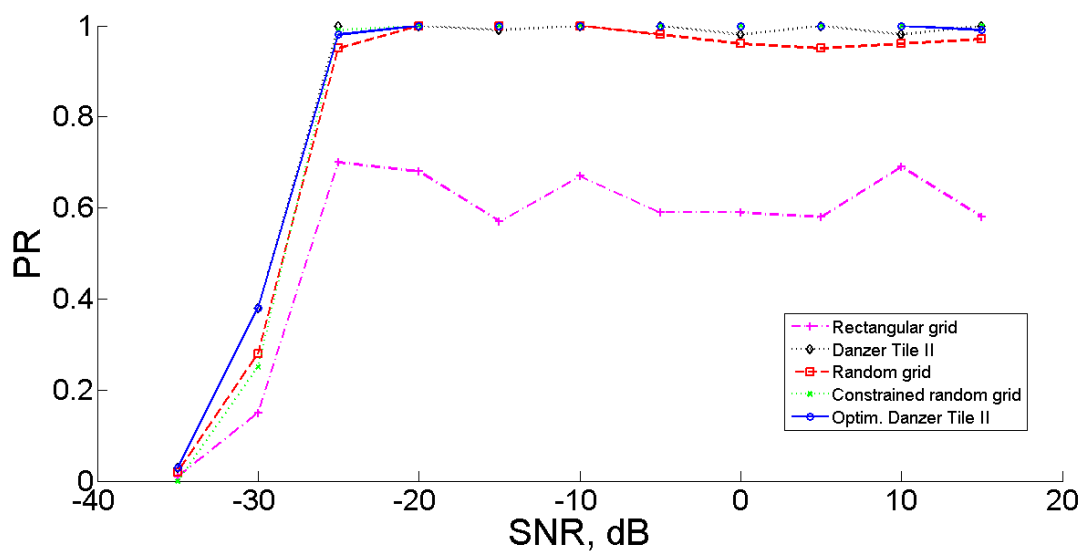

Figure 6.13: SNR vs the probability of resolution $\left(P_{R}\right)$ for Danzer Tile 2 aperture. 


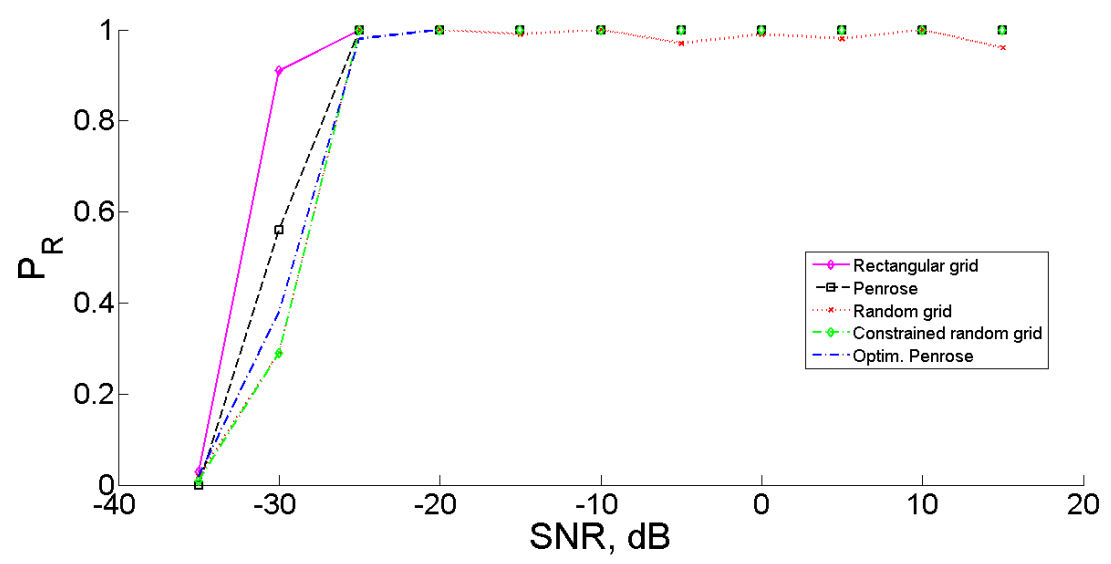

Figure 6.14: SNR vs the probability of resolution $\left(P_{R}\right)$ for Penrose Tile AL aperture.

Figure 6.13 shows the performance of different array configurations discussed in Section 6.4.2 based on "Danzer Geometry 2". Apart from the uniform grid geometry, the performance of other geometries is very similar. Optimal geometry still has a marginal advantage.

Figure 6.14 shows the performance of different array configurations based on "Penrose Geometry 1". The performance based on simulation parameters given in Table 6.2, is better for the uniform sampling configuration. In this case the optimum configure did not offer any advantage over the rest of the geometries. The performance, according to the MSE criterion, has a similar pattern. For "Danzer Geometry 1", Figure 6.15 shows the MSE vs SNR plots for the three targets given in Table 6.2, i.e., 60, 94, 144 degrees azimuth, with elevation kept at zero degree. For all the three targets, the MSE results of the optimal configuration are better than the rest of the configurations. Figure 6.16 shows the MSE vs SNR plots for "Danzer Geometry 2". In this case, for target 1 (78 degrees azimuth) and target 2 (94 degrees azimuth), the optimal configuration has better performance, while for 
target 3 (114 degrees azimuth), the constrained random configuration has slightly better performance than optimal configuration. In case of "Penrose Geometry 1", the performance of uniform sampling configuration is slightly better than the rest of the configurations. But the difference is not very significant, so we cannot make any strong conclusions about the results of "Penrose Geometry 1". 


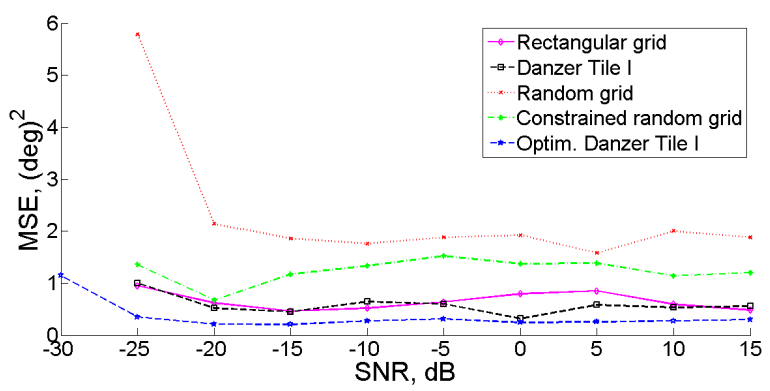

(a)

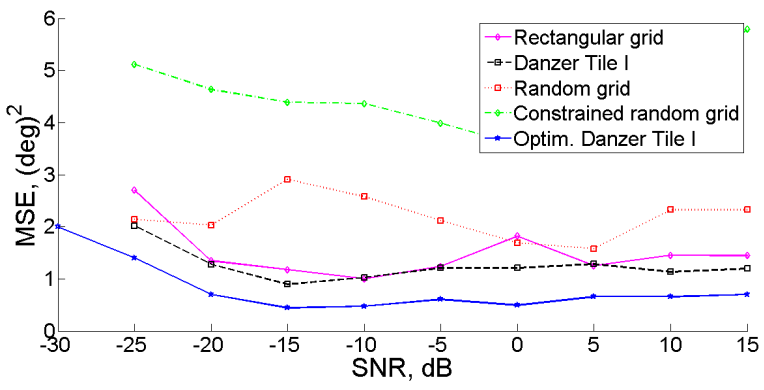

(b)

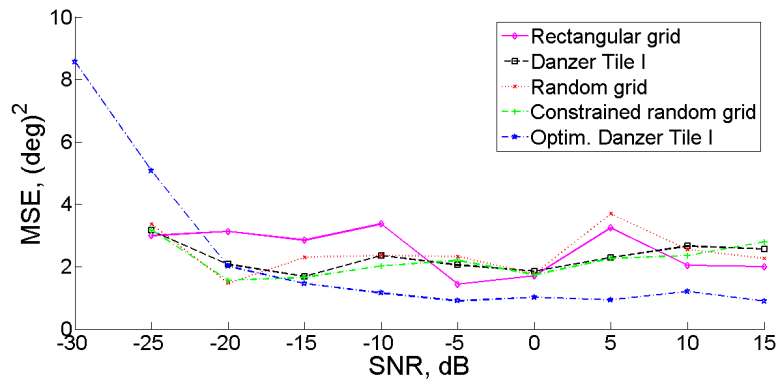

(c)

Figure 6.15: MSE plot for geometries based on Danzer Tile 1 aperture a) Target 1 at 60 degrees azimuth, b) Target 2 at 94 degrees azimuth, c) Target 3 at 144 degrees azimuth. Note that the vertical axis is not constant for the sub-figures. 


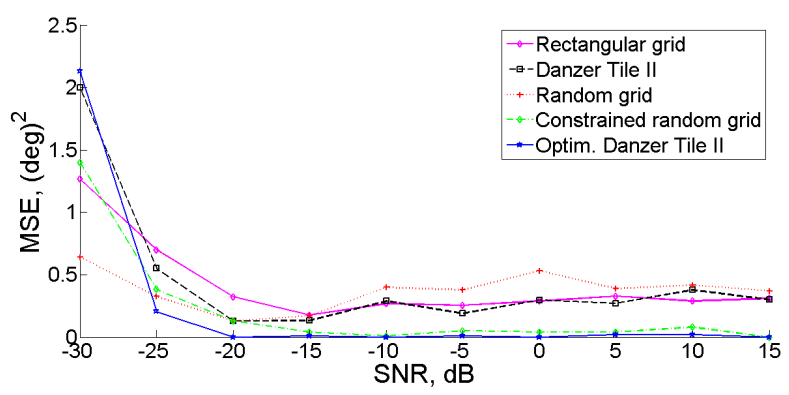

(a)

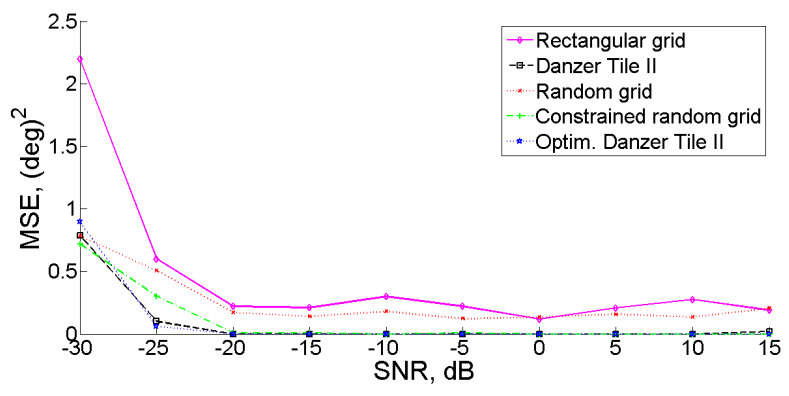

(b)

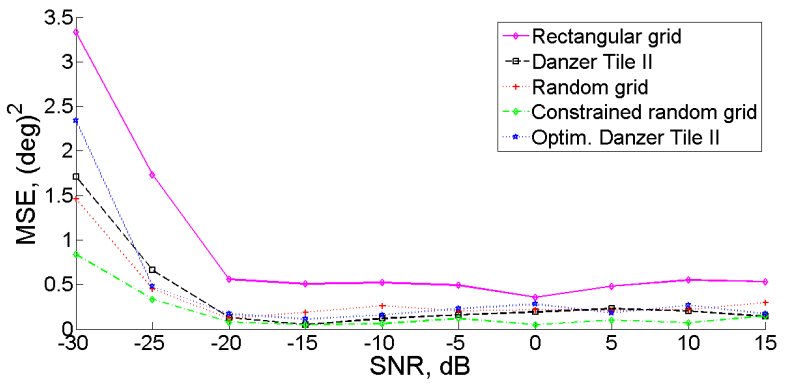

(c)

Figure 6.16: MSE plot for geometries based on Danzer Tile 2 aperture a) Target 1 at 78 degrees azimuth, b) Target 2 at 94 degrees azimuth, c) Target 3 at 114 degrees azimuth. Note that the vertical axis is not constant for the sub-figures. 


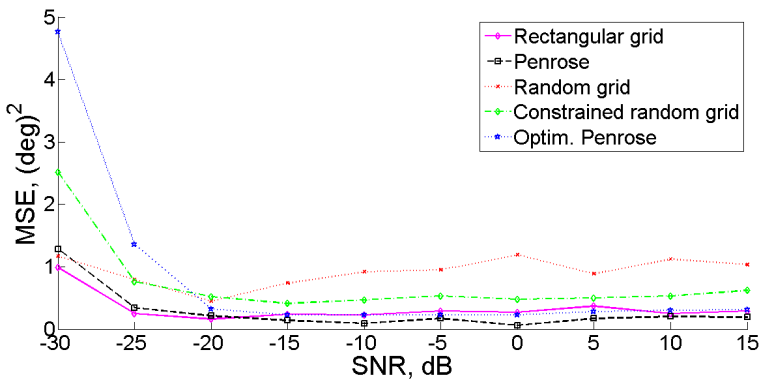

(a)

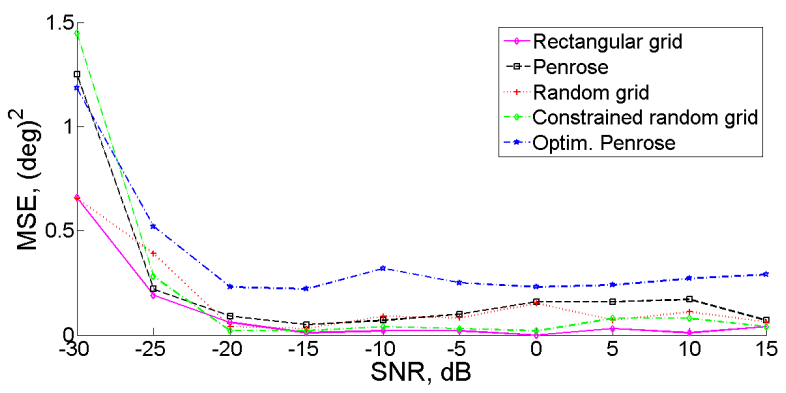

(b)

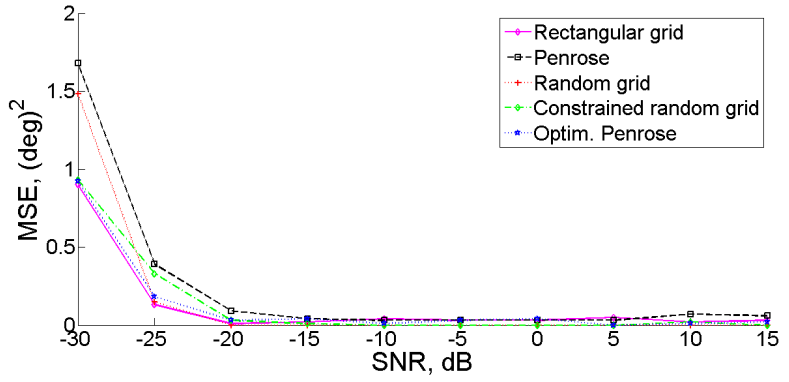

(c)

Figure 6.17: MSE plot for geometries based on Penrose Tile AL aperture a) Target 1 at 78 degrees azimuth, b) Target 2 at 94 degrees azimuth, c) Target 3 at 114 degrees azimuth. Note that the vertical axis is not constant for the sub-figures. 


\subsection{Conclusions}

Three sets of aperiodic geometries have been analyzed in this chapter. The array geometries were formed based on two types of aperiodic tiling namely Danzer tiling and Penrose tiling. The main motivation behind using these geometries has been to exploit the aperiodic structure of these geometries, as the aperiodic nature of these geometries have some advantages and are very useful when dealing with compressive sensing algorithms. The aperiodic geometry is well suited to the broadband algorithms (in classic no-CS scenario), as they avoid grating lobe problem, which create ambiguity in direction of arrival estimation. We have seen that using a Compressive Sensing algorithm such as broadband SOMP (Section 4.4) and using aperiodic geometry based on Danzer tiling we can achieve better performance in terms of MSE than using the uniformly sampled arrays, even when the aperture is kept constant. The uniformly sampled arrays use more elements than the aperiodic arrays, based on Danzer tiling and Penrose tiling. The aperiodic arrays are inherently sparse and we have used that fact to our advantage. The optimization formulation is valid for other aperiodic geometries such as fractal geometries. In a nutshell, any geometry that can be represented in $\boldsymbol{p}_{u}=\boldsymbol{T}_{u} \boldsymbol{p}$ form, where $\boldsymbol{p}$ contains initial set of points and $\boldsymbol{T}_{u}$ is a transformation matrix, we can apply the optimization problem and get a geometry that will perform better in the broadband and compressive sense. 


\section{Chapter 7}

\section{Conclusions and Future Works}

\subsection{Conclusions}

In Chapter 3, the main goal was to develop an algorithm that is capable of estimating the DOA of unknown number of sources with a very few data snapshots. The CS algorithms, like BPDN and OMP perform well in noiseless conditions and can recover severely undersampled signals. For noisy data, one snapshot is often not enough as multiple snapshots need to be integrated to improve signal to noise ratio and as a result improve the DOA estimation accuracy. MUSIC algorithm is a truly MMV algorithm, but it requires the information about the number of sources. SOMP, the MMV counterpart of the OMP algorithm, doesn't improve the estimation accuracy with increased number of snapshots, thus lacking in MMV integration capabilities, as compared to the MUSIC algorithm. We have developed a recursive algorithm, RML, that is a covariance based algorithm, similar to the MUSIC algorithm, with superior MMV integration capabilities as compared to SOMP. We 
have derived bounds for a parameter $\beta$, which is critical to the working of the RML algorithm, and thus eliminated the drawbacks that both MUSIC and MUSIC-like algorithms possess when encountered with a degenerate covariance matrix. The RML algorithm is recursive and estimates one source DOA at each iteration and removes the source signal from covariance matrix using subspace projections of the noise space. All these factors make the RML algorithm more general than the MUSIC and MUSIC-like algorithms, and much accurate than the SOMP algorithm as it has better MMV integration capabilities. We have shown by comparing the RML and SOMP algorithms using Montecarlo simulations, for different number of snapshots, that the performance of RML is comparable to SOMP when the snapshots are very few but superior to SOMP, when the number of snapshots are increased.

In Chapter 4, we extended the idea of the RML algorithm to broadband sources. The main obstacle we encounter, when using conventional algorithms like CSSM, is that the initial source estimates needs to be provided. Additionally, with a very few snapshots the covariance matrix can become degenerate, as a consequence, making the use of CSSM algorithm impractical. For low-SNR case, the inaccuracies in the initial estimate feed bias to the CSSM algorithm, which affects the final outcome and the bias makes the final result more inaccurate. Using focusing matrices approach, we developed an RML algorithm for broadband sources, that is capable of estimating unknown number of sources for the case when we have a very few snapshots and the covariance matrix is rank deficient. We also developed a broadband version of SOMP algorithm using the focusing matrices approach, as there is no known CS algorithm for the estimation of broadband DOA of unknown number of sources. Using simultaneous bearing-frequency focusing, we developed a new algorithm that is capable of estimating DOA of unknown number of sources for a very few snapshots. Using Montecarlo simulations, we saw that this algorithm is bet- 
ter suited to DOA estimation of broadband sources when the SNR conditions are good. We developed a Taylor series expansion based RML algorithm for broadband sources. All these algorithms were compared using Montecarlo simulations, where we compared probability of detection vs SNR for all the algorithms in compressive settings, when the snapshots were very few, as well as non-compressive settings. For large number of snapshots, we compared the four algorithms with CSSM and found that the broadband SOMP algorithm performed better than the rest of the algorithms. Derivative based RML algorithm, however, didn't converge for two sources even when the SNR was good but the snapshots were very few.

In Chapter 5, different geometry configurations were evaluated for their suitability to work with CS DOA estimation algorithm: BPDN. We developed a scheme to optimize geometry based on aperiodic tiling, with a perturbation method, so that the mutual coherence is minimized and we have exact recovery for sparse signals. The optimization scheme is general and can work with any geometry formed by translation, rotation and scaling of base tiles or shapes. It can be applied to any geometry based on aperiodic tiling and also to array geometries based on fractals. The aperiodic geometries are inherently sparse, and thus provide advantage in the number of sensor usage for a fixed aperture as compared to regular geometries. Using Montecarlo simulations we have shown that the optimized aperiod geometry based on Danzer tiling outperforms all other geometry configurations, with the only exception of highly impractical random geometry.

In Chapter 6, we have extended the ideas of Chapter 5 to broadband sources. Using Penrose tiling and Danzer tiling based geometries and using one of the algorithms (Broadband SOMP) developed in 4. Three sets of aperiodic geometries were analyzed, The main motivation behind using these geometries was to exploit the aperiodic structure of these geometries, as the aperiodic nature of these geometries 
have some advantages and are very useful when dealing with compressive sensing algorithms. The aperiodic tiling based array geometries are inherently sparse and by minimizing the mutual coherence, the individual elements of the optimized arrays are placed in such a way that the mutual coherence is minimized. Using Montecarlo simulations, we saw that the Danzer tiling based optimized geometry outperformed all other geometry configurations.

\section{2 $\quad$ Future Works}

In the future, the algorithm developed in Chapter 3 can be integrated with the OMP algorithm in such a way that the initial indices (initial source DOA estimates) can be provided through OMP algorithm while the remaining sources can be detected through RML algorithm. The reason is that the OMP algorithm is a single snapshot CS algorithm while RML algorithm still requires at least two snapshots to work properly (as a one snapshot covariance matrix would be rank one).

In future studies, the optimization scheme developed in Chapter 5, can be applied to other aperiodic geometries such as PinWheel tiling. The optimization scheme is also suitable for application to geometries based on fractals. It would be interesting to study the effects of the optimization scheme on the grating lobes and sidelobes for broadband antenna design. We have not studied this aspect as it is beyond the scope of this thesis.

An optimization scheme can be developed in future to optimize different parameters for sensor placements in coprime configuration [43]. In the Coprime Array with Compressed Inter-element Spacing (CACIS) discussed in the citation, the integer $\mathrm{p}$ used to compress the sub-array can be optimized using a scheme similar to the one 
discussed in Chapter 5. The coprime array, however, needs to be extended to two dimensional geometry to exploit the degrees of freedom available in the tranformation matrix for the optimization scheme (6.15). For broadband array design, the scheme, based on Taylor series expansion, developed by Shen et al [44] for off-grid DOA estimation can be used to extend the optimization scheme (6.15) to off-grid DOA estimation as well. 


\section{Author's Publications}

[C1] S. Z. Asghar and B. P. Ng, "Aperiodic geometry design for DOA estimation using compressive sensing" 2015 International Conference on Sampling Theory and Applications (SampTA), IEEE, DOI: 10.1109/SAMPTA.2015.7148942.

[J1] S. Z. Asghar and B. P. Ng, "Recursive MUSIC-like Algorithm for Estimation of DOA of Unknown Number of Sources," submitted to Multi Dimensional Systems and Signal Processing.

[J2] S. Z. Asghar and B. P. Ng, “Aperiodic geometry design for broadband DOA estimation using compressive sensing" submitted to IEEE Transactions on Signal Processing.

[J3] S. Z. Asghar and B. P. Ng, "Recursive MUSIC-like Class of Algorithms for Estimation of DOA of Unknown Number of Broadband Sources," manuscript under preparation. 


\section{Appendix A}

\section{Coordinate System}

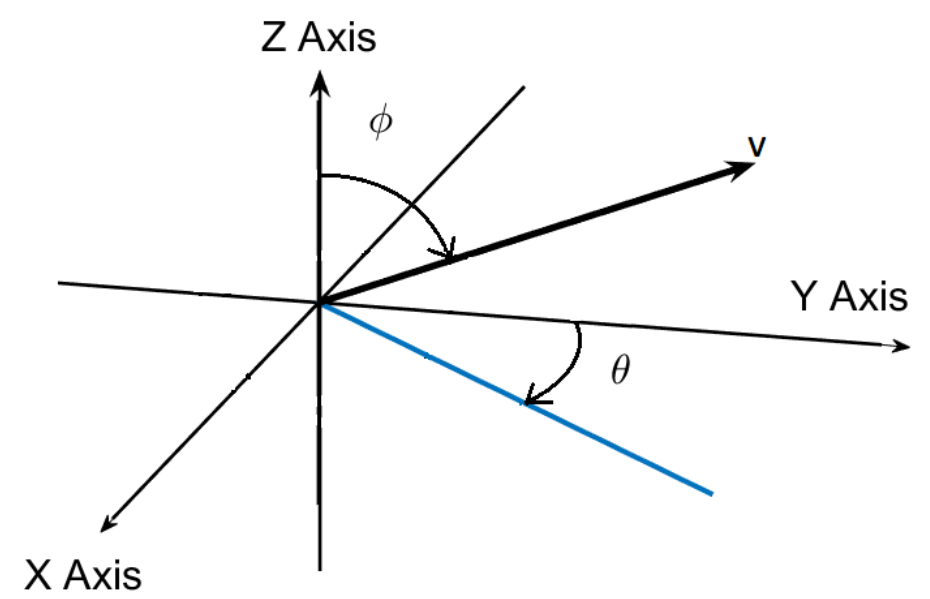

Figure A.1: The Coordinate System. $\theta$ is the azimuth angle, while $\phi$ is the polar angle.

Figure A.1 shows the coordinate system used throughout the thesis. Vector $\boldsymbol{v}$ is a three dimensional vector represented by Cartesian coordinates $(x, y, z)$. The 
figure also shows the azimuth angle, $\theta$ and the polar angle, $\phi$. The conversion between Cartesian and polar Coordinates is given below:

$$
\boldsymbol{v}=(r \cos \theta \sin \phi, r \sin \theta \sin \phi, r \cos \phi),
$$

where, $\theta \in(0,2 \pi]$ and $\phi \in[0, \pi]$ and $r=\sqrt{x^{2}+y^{2}+z^{2}}$.

The elevation angle, $\psi$, is given as:

$$
\psi=\frac{\pi}{2}-\phi,
$$

where, $\phi \in\left[\frac{-\pi}{2}, \frac{\pi}{2}\right]$. 


\section{Appendix B}

\section{Complex Orthogonal Procrustes}

\section{Problem}

According to the Greek mythology, Procrustes or Prokaptas was a smith and bandit, who physically attacked people by cutting their legs or stretching them as to fit the iron bed that he possessed.

In this problem [40], the matrix $\boldsymbol{A}$ is multiplied by another matrix $\boldsymbol{T}$ in order to bring it closer (or to fit) the matrix $\boldsymbol{B}$ :

$$
\begin{array}{r}
R=\quad \arg \left\{\min _{\boldsymbol{T}}\|\boldsymbol{T} \boldsymbol{A}-\boldsymbol{B}\|_{F}\right\}, \\
\text { subject to } \boldsymbol{T}^{H} \boldsymbol{T}=\boldsymbol{I}_{m} .
\end{array}
$$

The solution to this problem is:

$$
\boldsymbol{T}=\boldsymbol{U} \boldsymbol{V}^{T}
$$


where,

$$
\boldsymbol{U} \Sigma \boldsymbol{V}^{T}=\boldsymbol{B} \boldsymbol{A}^{T}
$$

\section{Proof}

Eq. B.2 can be written as,

$$
=\arg \left\{\min _{\boldsymbol{T}} . \operatorname{tr}\left\{\boldsymbol{A}^{T} \boldsymbol{A}\right\}+\operatorname{tr}\left\{\boldsymbol{B}^{T} \boldsymbol{B}\right\}-2 \operatorname{tr}\left\{\boldsymbol{B} \boldsymbol{A}^{T} \boldsymbol{T}^{T}\right\}\right\}
$$

which is equivalent to maximizing $\operatorname{tr}\left\{\boldsymbol{B} \boldsymbol{A}^{T} \boldsymbol{T}^{T}\right\}$. The maximizing matrix $\boldsymbol{T}$ can be found using Singular Value Decomposition (SVD) of $\boldsymbol{B} \boldsymbol{A}^{T}$, as given by (B.4).

$\operatorname{tr}\left\{\boldsymbol{B} \boldsymbol{A}^{T} \boldsymbol{T}^{T}\right\}$ can be written as following:

$$
\begin{aligned}
\operatorname{tr}\left\{\boldsymbol{B} \boldsymbol{A}^{T} \boldsymbol{T}^{T}\right\} & =\operatorname{tr}\left\{\boldsymbol{U} \boldsymbol{\Sigma} \boldsymbol{V}^{T} \boldsymbol{T}^{T}\right\}, \\
& =\operatorname{tr}\left\{\boldsymbol{U} \boldsymbol{T} \boldsymbol{V}^{T} \boldsymbol{\Sigma}\right\} \\
& =\operatorname{tr}\{\boldsymbol{Z} \boldsymbol{\Sigma}\}, \\
& =\sum_{i=1}^{m} z_{i i} \sigma_{i}<\sum_{i=1}^{m} \sigma_{i},
\end{aligned}
$$

where, $\boldsymbol{Z}=\boldsymbol{U} \boldsymbol{T} \boldsymbol{V}^{T}$, is an orthogonal matrix. The trace is maximized when $\boldsymbol{Z}=\boldsymbol{I}_{m}$ or $\boldsymbol{T}=\boldsymbol{U} \boldsymbol{V}^{T}$. This is the solution to the problem in (B.2). 


\section{Bibliography}

[1] H. L. V. Trees, "Optimum Array Processing: Part IV of Detection, Estimation, and Modulation Theory," John Wiley \& Sons, Inc., 2002.

[2] S. Haykin, "Array Signal Processing," Prentice Hall, 1984.

[3] P. S. Naidu, "Sensor Array Signal Processing," CRC Press, 2nd Edition, 2009.

[4] W. Roh et. al., "Millimeter-wave beamforming as an enabling technology for 5G cellular communications: theoretical feasibility and prototype results," IEEE Communications Magazine, vol. 52, no. 2, pp 106-113, 2014.

[5] J.C. Maxwell, "A Dynamical Theory of the Electromagnetic Field," Philosophical Transactions of the Royal Society of London, 1865.

[6] H. Akaike, "A new look at the statistical model identification" IEEE Trans. Automatic Control, vol. 19, no. 6, pp. 716-723, Dec. 1974.

[7] J. Rissanen, "Modeling by shortest data description," Automatica, vol. 14, no. 5, pp. 465-471, Sep. 1978.

[8] J. Capon, "High-resolution frequency-wavenumber spectrum analysis" Proc. of the IEEE, vol. 57, no. 8, pp. 1408-1418, Jun. 1969. 
[9] E. Cand'es, J. Romberg, and T. Tao, "Robust uncertainty principles: Exact signal reconstruction from highly incomplete frequency information" IEEE Trans. Inform. Theory, vol. 52, no. 2, pp. 489-509, 2006.

[10] E. Cand'es and T. Tao,"Near optimal signal recovery from random projections: Universal encoding strategies?" IEEE Trans. Inform. Theory, vol. 52, no. 12, pp. 5406-5425, 2006.

[11] D. Donoho, "Compressed sensing," IEEE Trans. Inform. Theory, vol. 52, no. 4, 2006.

[12] E. Cand'es, J. Romberg, and T. Tao, "Stable signal recovery from incomplete and inaccurate measurements" Comm. Pure Appl. Math., vol. 59, no. 8, pp. 1207-1223, 2006.

[13] E.J. Candes, M.B. Wakin, "An Introduction To Compressive Sampling," IEEE Signal Processing Magazine, Vol. 25, No. 2, pp 21 - 30 Mar 2008.

[14] D. L. Donoho. M. Elad. and V. Temlyakov, "Stable recovery of sparse overcomplete representation in the presence of noise," IEEE Transactions on Information Theory, Vol. 52, No. 1, pp. 6-18, Jan 2006.

[15] J. A. Tropp, "Greed is good:Algorithmic results for sparse approximation," IEEE Transaction Information Theory, Vol. 50, pp 2231-2242, Oct 2004.

[16] J. A. Tropp, A. C. Gilbert and M. J. Strauss, "Algorithms for simultaneous sparse approximation. Part I: Greedy Pursuit," Signal Processing, Vol. 86, No. 3, pp 572-588, Mar 2006. 
[17] J. Chen and X. Huo, "Theoretical results on sparse representations of multiplemeasurement vectors," IEEE Transactions on Signal Processing, Vol. 54, No 12, pp 4634-4643, Dec 2006.

[18] T.G. Spence, and D.H. Werner, "Design of Broadband Planar Arrays Based on the Optimization of Aperiodic Tilings," IEEE Transactions on Antennas and Propagation, Vol. 56, No. 1, pp. 76 - 86, Jan 2008.

[19] K.-P. Nischke and L. Danzer , "A construction of inflation rules based on n-fold symmetry," Discrete \& Computational Geometry Vol. 15, No 2 , pp 221-236, Feb 1996.

[20] Emmanuel Cand'es and Justin Romberg, "Sparsity and incoherence in compressive sampling," Inverse Problems, Vol. 23, No. 3, 2007.

[21] M. A. Herman, T. Strohmer, "High-Resolution Radar via Compressed Sensing," IEEE Transactions on Signal Processing, Vol. 57 , No. 6, pp 2275 - 2284, Feb 2009.

[22] J. H. G. Ender, "On compressive sensing applied to radar," Signal Processing, Vol 90, pp 1402-1414, 2010.

[23] Paul J. Steinhart, "Quasicrystals: a breif history of the impossible," Rendiconti Lincei, Vol 24, No. 1 Supplement, pp 85-91, Mar 2013.

[24] D. H. Werner and S. Ganguly, "An overview of fractal antenna engineering research," IEEE Antennas Propagation Magazine, Vol 45, No. 1, pp 38-57, Feb 2003. 
[25] J. M. Kim, O. K. Lee and J. C. Ye "Compressive MUSIC: Revisiting the link between Compressive Sensing and Array Signal Processing", pp. 278-301, IEEE Transactions on Information Theory, vol 58, No 1, January 2012.

[26] M. Mishali and Y. C. Eldar, "Reduce and boost: Recovering arbitrary sets of jointly sparse vectors," IEEE Trans. Signal Process., vol. 56, no. 10, pp. 4692-4702, 2008.

[27] Kiryung Lee and Yoram Bresler, "Subspace-augmented MUSIC for joint sparse recovery with any rank", IEEE Sensor Array and Multichannel Signal Processing Workshop, 2010.

[28] Ying Zhang and Boon Poh Ng, "MUSIC-Like DOA Estimation Without Estimating the Number of Sources", pp. 1668-1676, IEEE Transactions on Signal Processing, vol. 58, no. 3, March 2010.

[29] R. O. Schmidt, "Multiple emitter location and signal parameter estimation," IEEE Transanctions on Antennas and Propagation, vol. 34, no. 3, pp. 276-280, Mar. 1986.

[30] R. Roy and T. Kailath, "ESPRIT-estimation of signal parameters via rotational invariance techniques," IEEE Transactions on Acoustics, Speech and Signal Processing, vol. 37, no. 7, pp. 984-995, 1989.

[31] V.V. Reddy, B.P. Ng, A.W.H. Khong, "Insights Into MUSIC-Like Algorithm," Signal Processing, IEEE Transactions on, vol. 61 ,no. 10, pp. 2551-2556, Mar. 2013. 
[32] Y. Bresler, "Spectrum-blind sampling and compressive sensing for continuousindex signals," Proc. IEEE Information Theory and Applications Workshop, pp. 547-554, 2008.

[33] B.P. Ng, M.H.Er and C. Kot. "A MUSIC approach for estimation of directions of arrival of multiple narrowband and broadband sources," Signal Processing, vol. 40, nos. 2-3, pp. 319-323, 1994.

[34] M. Wax, T. Shan, and T. Kailath, "Spatio-temporal spectral analysis by eigenstructure methods," IEEE Transactions on Acoustics, Speech and Signal Processing, vol. 32, no. 4, pp. 817-827, 1984.

[35] Vinod V. Reddy, B.P. Ng and Andy W. H. Khong, "Derivative-constrained frequency-domain wideband DOA estimation", Multidimensional Systems and Signal Processing, vol 25, no 1, pp 211-233, 2014.

[36] F. Sellone, "Robust auto-focusing wideband DOA estimation", Signal Processing, vol 86, no 1, pp 17-37, 2006.

[37] H. L. V. Trees, Optimum Array Processing. Wiley, 2002.

[38] G. E. Owen, Introduction to Electromagnetic Theory, Dover Books, 2003.

[39] H. Hung and M. Kaveh,"Focussing matrices for coherent signal-subspace processing," IEEE Trans. Acoustics, Speech and Signal Process., vol. 36, no. 8, pp. 1272-1281, 1988.

[40] P.H. Schonemann, "A generalized solution of the orthogonal Procrustes problem," Psychometrika, vol. 31, pp 1-10, 1966. 
[41] W.-K. Ma, T.-H. Hsieh, and C.-Y. Chi, "DOA estimation of quasi-stationary signals with less sensors than sources and unknown spatial noise covariance: A Khatri-Rao subspace approach," IEEE Trans. Antennas Propagat., vol. 58, no. 4, pp. 2168-2180, 2010.

[42] P. Pal, P. P. Vaidyanathan, "Nested Arrays: A Novel Approach to Array Processing With Enhanced Degrees of Freedom," IEEE Transactions on Signal Processing, vol. 58, no. 8, pp 4167 - 4181, 2010.

[43] S. Qin, Y. D. Zhang, and M. G. Amin, "Generalized co-prime array configurations for direction-of-arrival estimation," IEEE Transactions on Signal Processing, vol. 63, no. 6, pp. 1377-1390, March 2015.

[44] Q. Shen, W. Liu, W. Cui, S. Wu, Y. D. Zhang, and M. G. Amin, "Underdetermined Wideband DOA Estimation of Off-Grid Sources Employing the Difference Co-Array Concept," IEEE Signal Processing Letters, vol. 130, pp. 299-304, January 2017.

[45] D. H. Werner, R. L. Haupt, and P. L. Werner, "Fractal antenna engineering: The theory and design of fractal antenna arrays," IEEE Antennas Propag. Mag., vol. 41, no. 5, pp. 37-59, 1999. 